\title{
Abelian Conformal Field Theory and Determinant Bundles*
}

\author{
Jørgen Ellegaard Andersen \& Kenji Ueno
}

\begin{abstract}
Following KNTY we study a so-called $b c$-ghost system of zero conformal dimension from the viewpoint of [TUY] and [U2. We show that the ghost vacua construction results in holomorphic line bundles with connections over holomorphic families of curves. We prove that the curvature of these connections are up to a scale the same as the curvature of the connections constructed in [TUY] and [U2]. We study the sewing construction for nodal curves and its explicit relation to the constructed connections. Finally we construct preferred holomorphic sections of these line bundles and analyze their behaviour near nodal curves. These results are used in AU2 to construct modular functors form the conformal field theories given in TUY] and [U2] by twisting with an appropriate factional power of this Abelian theory.
\end{abstract}

\section{Contents}

1 Fermion Fock space

2 Universal Grassmann Manifold 8

3 Ghost Vacua 11

4 Sheaf of Ghost Vacua 24

5 Degeneration of Curves and Sewing 35

6 Formal Coordinates and Preferred Sections 52

\section{Introduction}

The present paper is the first in a series of three papers ([AU2] and [AU3]), in which we shall construct modular functors and the Reshetikhin-Turaev Topological Quantum Field Theories from the conformal field theories developed in [TUY] and [U2].

The basic idea behind the construction of a modular functor for a simple Lie algebra is the following. The sheaf of vacua construction for a simple Lie algebra gives a vector bundle with connection over Teichmüller space of any oriented pointed surface. The vector

\footnotetext{
${ }^{*}$ This research was conducted partly by the first author for the Clay Mathematics Institute at University of California, Berkeley and for MaPhySto - Centre for Mathematical Physics and Stochastics, funded by The Danish National Research Foundation. The second author is partially supported by Grant in Aid for Scientific Research NO. 14102001 of JSPS.
} 
space that the modular functor associates to the oriented pointed surface should be the covariant constant sections of the bundle. However the connection on the bundle is only projectively flat, so we need to find a suitable line bundle with a connection, such that the tensor product of the two has a flat connection.

We shall construct a line bundle with a connection on any family of $N$-pointed curves with formal coordinates. By computing the curvature of this line bundle, we conclude that we actually need a fractional power of this line bundle so as to obtain a flat connection after tensoring. In order to functorially extract this fractional power, we need to construct a preferred section of the line bundle.

We shall construct the line bundle by the use of the so-called $b c$-ghost systems (FaddeevPopov ghosts) first introduced in covariant quantization [FP]. The $b c$ system have two anticommuting fields $b(z), c(z)$ of conformal dimension $j, 1-j$, respectively, where $j$ is an integer or half integer. In the case $j=1 / 2$ a mathematically rigorous treatment was given in the paper [KNTY]. The case $j=1 / 2$ corresponds to the study of the determinant bundle of half-canonical line bundles on smooth curves, i.e.. on compact Riemann surfaces. Since we cannot define the half-canonical line bundles for curves with node, whose normalization has at least two components, the boundary behavior of the sheaf of vacua is complicated [KSUU]. Therefore, in the present paper we shall consider the case $j=0$, following the ideas of [KNTY], but describing it from the viewpoint of [TUY]. We shall also use the terminology of [TUY] and [U2. In particular we shall define the sheaf of ghost vacua on a family of $N$-pointed Riemann surfaces with formal coordinates and we shall introduce a connection on it. All the necessary properties which we need to construct for our modular functor construction in AU2 will be proved in this paper. The sheaf of the ghost vacua is isomorphic to the invertible sheaf associated to the determinant bundle of the relative canonical sheaf of the family.

Let us explain briefly the contents of the present paper. In section 1 we shall introduce the fermion operators and the fermion Fock space and fix the notation which will be used in the present paper.

In section 2 the universal Grassmann manifold due to M. Sato will be defined. The universal Grassmann manifold is an infinite dimensional manifold which can be embedded into an infinite dimensional projective space. The pull-back of the hyperplane line bundle of the projective space is the determinant bundle of the universal subbundle of the universal Grassmann manifold.

In section 3 we shall develop the theory of $j=0$ ghost systems. The main purpose of this section is to define the space of ghost vacua for an $N$-pointed curve with formal coordinates. Also basic properties of the space of ghost vacua will be discussed and we proved important theorems such as propagation of vacua. The space of ghost vacua is a one-dimensional vector space. This will be proved in section 4 and section 5 .

In section 4 we shall consider a family of $N$-pointed curves with formal coordinates and define the sheaf of ghost vacua attached to the family. The projectively flat connection will be defined on the sheaf of ghost vacua.

In section 5 we shall consider smoothings of nodal curves. In particular we shall construct a section of the sheaf of ghost vacua starting from an element of the ghost vacua of the nodal curve. This construction is called sewing and it is the key to proving that the space ghost vacua is a one-dimensional vector space.

In section 6 we shall construct the preferred element of an $N$-pointed curve with formal 
coordinates and study its basic properties.

\section{Fermion Fock space}

Let $\mathbf{Z}_{h}$ be the set of all half integers. Namely

$$
\mathbf{Z}_{h}=\{n+1 / 2 \mid n \in \mathbf{Z}\} .
$$

Let $\mathcal{W}^{\dagger}$ be an infinite-dimensional vector space over $\mathbf{C}$ with a filtration $\left\{F^{m} \mathcal{W}^{\dagger}\right\}_{m \in \mathbf{Z}}$ which satisfies the following conditions.

1. The filtration $\left\{F^{m} \mathcal{W}^{\dagger}\right\}$ is decreasing;

2. $\bigcup_{m \in \mathbf{Z}} F^{m} \mathcal{W}^{\dagger}=\mathcal{W}^{\dagger}, \quad \bigcap_{m \in \mathbf{Z}} F^{m} \mathcal{W}^{\dagger}=\{0\} ;$

3. $\operatorname{dim}_{\mathbf{C}} F^{m} \mathcal{W}^{\dagger} / F^{m+1} \mathcal{W}^{\dagger}=1$;

4. The vector space $\mathcal{W}^{\dagger}$ is complete with respect to the uniform topology such that $\left\{F^{m} \mathcal{W}^{\dagger}\right\}$ is a basis of open neighbourhoods of 0 .

We introduce a basis $\left\{e^{\nu}\right\}_{\nu \in \mathbf{Z}_{h}}$ of $\mathcal{W}^{\dagger}$ in such a way that

$$
e^{m+1 / 2} \in F^{m} \mathcal{W}^{\dagger} \backslash F^{m+1} \mathcal{W}^{\dagger}
$$

Then, each element $u \in \mathcal{W}^{\dagger}$ can uniquely be expressed in the form

$$
u=\sum_{\nu>n_{0}, \nu \in \mathbf{Z}_{h}}^{\infty} a_{\nu} e^{\nu}
$$

for some $n_{0}$ and with respect to this basis the filtration is given by

$$
F^{m} \mathcal{W}^{\dagger}=\left\{u \in \mathcal{W}^{\dagger} \mid u=\sum_{\nu>m, \nu \in \mathbf{Z}_{h}}^{\infty} a_{\nu} e^{\nu}\right\}
$$

We fix the basis $\left\{e^{\nu}\right\}_{\nu \in \mathbf{Z}_{h}}$ throughout the present paper.

Let $\mathbf{C}((\xi))$ be a field of formal Laurent series over the complex number field. Then the basis gives us a filtration preserving linear isomorphism

$$
\begin{aligned}
\mathbf{C}((\xi)) & \cong \mathcal{W}^{\dagger} \\
\xi^{n} & \mapsto e^{n+1 / 2} .
\end{aligned}
$$

By mapping $\xi^{n} d \xi$ to $e^{n+1 / 2}$ we of course also get a filtration preserving linear isomorphism between $\mathbf{C}((\xi)) d \xi$ and $\mathcal{W}^{\dagger}$.

We let $\left\{\bar{e}_{\nu}\right\}_{\nu \in \mathbf{Z}_{h}}$ be the dual basis of $\left\{e^{\nu}\right\}_{\nu \in \mathbf{Z}_{h}}$. Then, put

$$
\mathcal{W}=\bigoplus_{\nu \in \mathbf{Z}_{h}} \mathbf{C} \bar{e}_{\nu}
$$




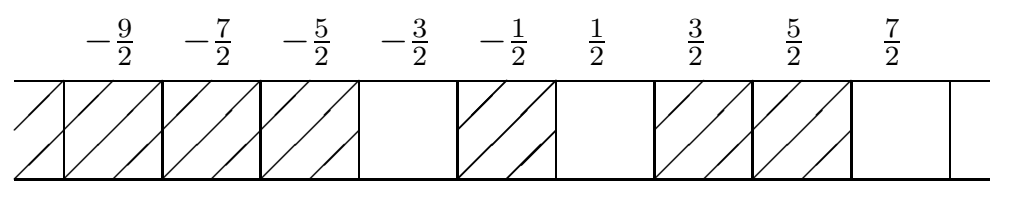

$$
\mu(\nu)=\nu \text { for } \nu \leq-\frac{5}{2}, \mu\left(-\frac{3}{2}\right)=-\frac{1}{2}, \mu\left(-\frac{1}{2}\right)=\frac{2}{3}, \mu\left(\frac{1}{2}\right)=\frac{5}{2}
$$

Figure 1: Maya diagram of charge 1 and degree 5

Then $\mathcal{W}$ is the topological dual of the vector space $\mathcal{W}^{\dagger}$. There is a natural pairing $(\quad \mid \quad)$ : $\mathcal{W}^{\dagger} \times \mathcal{W} \rightarrow \mathbf{C}$ defined by

$$
\left(e^{\nu} \mid \bar{e}_{\mu}\right)=\delta_{\mu}^{\nu}
$$

In other word we have

$$
(u \mid v)=v(u) .
$$

The complete topological vector space $\mathcal{W}^{\dagger}$ will be used in the next section to define the universal Grassmann manifold due to M. Sato ([SA]).

Here let us introduce the semi-infinite exterior product of the vector spaces $\mathcal{W}$ and $\mathcal{W}^{\dagger}$. For that purpose we first introduce the notion of a Maya diagram.

Definition 1.1. A Maya diagram $M$ of the charge $p, p \in \mathbf{Z}$ is a set

$$
M=\{\mu(p-1 / 2), \mu(p-3 / 2), \mu(p-5 / 2), \ldots\},
$$

where $\mu$ is an increasing function

$$
\mu: \mathbf{Z}_{h<p}=\left\{\nu \in \mathbf{Z}_{h} \mid \nu<p\right\} \rightarrow \mathbf{Z}_{h}
$$

such that there exists an integer $n_{0}$ such that

$$
\mu(\nu)=\nu
$$

for all $\nu<n_{0}$.

The function $\mu$ is called the characteristic function of the Maya diagram $M$. The set of Maya diagrams of charge $p$ is written as $\mathcal{M}_{p}$.

A Maya diagram $M$ of charge $p$ with its characteristic function $\mu$ can be expressed by a diagram as in Figure 1.

For a Maya diagram M we have $\mu(\nu)=\nu$ for almost all $\nu$. Therefore the set

$$
\left\{\mu(\nu)-\nu \mid \nu \in \mathbf{Z}_{h}, \mu(\nu)-\nu>0\right\}
$$

is finite and the number

$$
d(M)=\sum_{\nu \in \mathbf{Z}_{h}}(\mu(\nu)-\nu)
$$


is finite. The number $d(M)$ is also written as $d(\mu)$ and it is called the degree of the Maya diagram $M$ with characteristic function $\mu$. The finite set of Maya diagrams of degree $d$ and change $p$ is denoted $\mathcal{M}_{p}^{d}$. Clearly $\mathcal{M}_{p}=\coprod_{d} \mathcal{M}_{p}^{d}$.

For a Maya diagram $M$ of charge $p$ we define two semi-infinite products

$$
\begin{aligned}
|M\rangle & =\bar{e}_{\mu(p-1 / 2)} \wedge \bar{e}_{\mu(p-3 / 2)} \wedge \bar{e}_{\mu(p-5 / 2)} \wedge \cdots \\
\langle M| & =\cdots \wedge e^{\mu(p-5 / 2)} \wedge e^{\mu(p-3 / 2)} \wedge e^{\mu(p-1 / 2)}
\end{aligned}
$$

Formally, these semi-infinite products is just another notation for the corresponding Maya diagram. This notation is particular convenient for the following discussion. However, by using the basis $e_{\nu}$, we clear indicate the relation to the vector spaces $\mathcal{W}$ and $\mathcal{W}^{\dagger}$.

For any integer $p$ put

$$
\begin{aligned}
& |p\rangle=\bar{e}_{p-1 / 2} \wedge \bar{e}_{p-3 / 2} \wedge \bar{e}_{p-5 / 2} \wedge \cdots \\
& \langle p|=\cdots \wedge e^{p-5 / 2} \wedge e^{p-3 / 2} \wedge e^{p-1 / 2}
\end{aligned}
$$

Now the fermion Fock space $\mathcal{F}^{\dagger}(p)$ of charge $p$ and the dual fermion Fock space $\mathcal{F}(p)$ of charge $p$ are defined by

$$
\begin{aligned}
\mathcal{F}(p) & =\bigoplus_{M \in \mathcal{M}_{p}} \mathbf{C}|M\rangle \\
\mathcal{F}^{\dagger}(p) & =\prod_{M \in \mathcal{M}_{p}} \mathbf{C}\langle M|
\end{aligned}
$$

We observe that

$$
\mathcal{F}(p)=\bigoplus_{d \geq 0} \mathcal{F}_{d}(p)
$$

where

$$
\mathcal{F}_{d}(p)=\bigoplus_{M \in \mathcal{M}_{p}^{d}} \mathbf{C}|M\rangle
$$

The dual pairing

$$
\langle\cdot \mid \cdot\rangle: \mathcal{F}^{\dagger}(p) \times \mathcal{F}(p) \rightarrow \mathbf{C}
$$

is given by

$$
\langle M \mid N\rangle=\delta_{M, N}, \quad M, N \in \mathcal{M}_{p}
$$

Put also

$$
\begin{aligned}
\mathcal{F} & =\bigoplus_{p \in \mathbf{Z}} \mathcal{F}(p) \\
\mathcal{F}^{\dagger} & =\bigoplus_{p \in \mathbf{Z}} \mathcal{F}^{\dagger}(p)
\end{aligned}
$$

The vector space $\mathcal{F}^{\dagger}$ is called the fermion Fock space and $\mathcal{F}$ is called the dual fermion Fock space. These are the semi-infinite exterior products of the vector spaces $\mathcal{W}^{\dagger}$ and $\mathcal{W}$ respectively, which we shall be interested in. We only define the fermion Fock space by using the basis $e_{\nu}$, since we are fixing this basis throughout. 
The above pairing can be extended to the one on $\mathcal{F}^{\dagger} \times \mathcal{F}$ by assuming that the paring is zero on $\mathcal{F}^{\dagger}(p) \times \mathcal{F}\left(p^{\prime}\right)$ if $p \neq p^{\prime}$.

Let us introduce the fermion operators $\psi_{\nu}$ and $\bar{\psi}_{\nu}$ for all half integers $\nu \in \mathbf{Z}_{h}$ which act on $\mathcal{F}$ from the left and on $\mathcal{F}^{\dagger}$ from the right.

$$
\begin{aligned}
\text { Left action on } \mathcal{F} & \psi_{\nu}=i\left(\bar{e}_{\nu}\right), \quad \bar{\psi}_{\nu}=\bar{e}_{-\nu} \wedge \\
\text { Right action on } \mathcal{F}^{\dagger} & \psi_{\nu}=\wedge e^{\nu}, \quad \bar{\psi}_{\nu}=i\left(e^{-\nu}\right)
\end{aligned}
$$

where $i(\cdot)$ is the interior product. For example we have

$$
\begin{aligned}
\psi_{-3 / 2}|0\rangle & =i\left(\bar{e}_{-3 / 2}\right) \bar{e}_{-1 / 2} \wedge \bar{e}_{-3 / 2} \wedge \cdots=-\bar{e}_{-1 / 2} \wedge \bar{e}_{-5 / 2} \wedge \bar{e}_{-7 / 2} \wedge \cdots, \\
\langle 0| \bar{\psi}_{5 / 2} & =\cdots \wedge e^{-5 / 2} \wedge e^{-3 / 2} \wedge e^{-1 / 2} i\left(e^{-5 / 2}\right)=\cdots \wedge e^{-7 / 2} \wedge e^{-3 / 2} \wedge e^{-1 / 2}
\end{aligned}
$$

Note that $\psi_{\nu}$ maps $\mathcal{F}(p)$ to $\mathcal{F}(p-1)$, hence decreases the charge by one, and $\bar{\psi}_{\nu}$ maps $\mathcal{F}(p)$ to $\mathcal{F}(p+1)$, hence increase the charge by one. Similarly the right action of $\psi_{\nu}$ maps $\mathcal{F}^{\dagger}(p)$ to $\mathcal{F}^{\dagger}(p+1)$ and $\bar{\psi}_{\nu}$ maps $\mathcal{F}^{\dagger}(p)$ to $\mathcal{F}^{\dagger}(p-1)$. It is easy to show that for any $\langle u| \in \mathcal{F}$ and $|v\rangle \in \mathcal{F}^{\dagger}$ we have

$$
\left\langle u \mid \psi_{\nu} v\right\rangle=\left\langle u \psi_{\nu} \mid v\right\rangle, \quad\left\langle u \mid \bar{\psi}_{\nu} v\right\rangle=\left\langle u \bar{\psi}_{\nu} \mid v\right\rangle .
$$

Also it is easy to show that

$$
\begin{array}{ll}
\psi_{\nu}|0\rangle=0 & \text { if and only if } \nu>0 \\
\bar{\psi}_{\nu}|0\rangle=0 & \text { if and only if } \nu>0
\end{array}
$$

Similarly we have

$$
\begin{array}{ll}
\langle 0| \psi_{\nu}=0 & \text { if and only if } \nu<0, \\
\langle 0| \bar{\psi}_{\nu}=0 & \text { if and only if } \nu<0 .
\end{array}
$$

The fermion operators have the following anti-commutation relations as operators on $\mathcal{F}$ and $\mathcal{F}^{\dagger}$.

$$
\begin{aligned}
{\left[\psi_{\nu}, \psi_{\mu}\right]_{+} } & =0 \\
{\left[\bar{\psi}_{\nu}, \bar{\psi}_{\mu}\right]_{+} } & =0 \\
{\left[\psi_{\nu}, \bar{\psi}_{\mu}\right]_{+} } & =\delta_{\nu+\mu, 0},
\end{aligned}
$$

where we define

$$
[A, B]_{+}=A B+B A .
$$

Note that for each Maya diagram $M$ of charge $p$ we can find non-negative half integers

$$
\mu_{1}<\mu_{2}<\cdots<\mu_{r}<0, \quad \nu_{1}<\nu_{2}<\cdots<\nu_{s}<0, \quad r \geq 0, s \geq 0
$$

with $r-s=p$ and $\mu_{i} \neq \nu_{j}$ such that

$$
|M\rangle=(-1)^{\sum_{i=1}^{s} \nu_{i}+s / 2} \bar{\psi}_{\mu_{1}} \bar{\psi}_{\mu_{2}} \cdots \bar{\psi}_{\mu_{r}} \psi_{\nu_{s}} \psi_{\nu_{s-1}} \cdots \psi_{\nu_{1}}|0\rangle
$$


The negative half integers $\mu_{i}$ 's and $\nu_{j}$ 's are uniquely determined by the Maya diagram $M$. The normal ordering : : of the fermion operators are defined as follows.

$$
: A_{\nu} B_{\mu}:= \begin{cases}-B_{\mu} A_{\nu} & \text { if } \mu<0 \text { and } \nu>0 \\ A_{\nu} B_{\mu} & \text { otherwise }\end{cases}
$$

where $A$ and $B$ is $\psi$ or $\bar{\psi}$. By (1.3), (1.4) and (1.5) the normal ordering is non-trivial if and only if $\mu<0$ and $A_{\nu}=\bar{B}_{-\mu}$. For example we have

$$
: \bar{\psi}_{1 / 2} \psi_{-1 / 2}:=-\psi_{-1 / 2} \bar{\psi}_{1 / 2} \text {, }
$$

although as operators on $\mathcal{F}$ or $\mathcal{F}^{\dagger}$ we have

$$
\bar{\psi}_{1 / 2} \psi_{-1 / 2}=-\psi_{-1 / 2} \bar{\psi}_{1 / 2}+\mathrm{Id} .
$$

Thus the normal ordering has the effect of subtracting the identity operator, whenever it is non-trivial.

The field operators $\psi(z)$ and $\bar{\psi}(z)$ are defined by

$$
\begin{aligned}
\psi(z) & =\sum_{\mu} \psi_{\mu} z^{-\mu-1 / 2}, \\
\bar{\psi}(z) & =\sum_{\mu} \bar{\psi}_{\mu} z^{-\mu-1 / 2} .
\end{aligned}
$$

The current operator $J(z)$ is defined by

$$
J(z)=: \bar{\psi}(z) \psi(z):=\sum_{n \in \mathbf{Z}} J_{n} z^{-n-1}
$$

Note that thanks to the normal ordering, the operator $J_{n}$ can operate on $\mathcal{F}$ and $\mathcal{F}^{\dagger}$ even though $J_{n}$ is an infinite sum of operators.

For any integer or half integer $j$ the energy-momentum tensor $T^{(j)}(z)$ is defined by

$$
T^{(j)}(z)=:(1-j) \frac{d \psi(z)}{d z} \bar{\psi}(z)-j \psi(z) \frac{d \bar{\psi}(z)}{d z}:=\sum_{n \in \mathbf{Z}} L_{n}^{(j)} z^{-n-2} .
$$

Again due to the normal ordering, the coefficients $L_{n}^{(j)}$ operates on $\mathcal{F}$ and $\mathcal{F}^{\dagger}$.

These operators satisfy the following commutation relations.

$$
\begin{aligned}
{\left[J_{n}, J_{m}\right] } & =n \delta_{n+m, 0} \\
{\left[L_{n}^{(j)}, L_{m}^{(j)}\right] } & =(n-m) L_{n+m}^{(j)}-\frac{1}{6}\left(6 j^{2}-6 j+1\right)\left(n^{3}-n\right) \delta_{m+n .0} \\
{\left[L_{n}^{(j)}, J_{m}\right] } & =-m J_{n+m}-\frac{1}{2}(2 j-1)\left(n^{2}+n\right) \delta_{n+m, 0} \\
{\left[J_{n}, \psi(z)\right] } & =-z^{n} \psi(z) \\
{\left[J_{n}, \bar{\psi}(z)\right] } & =z^{n} \bar{\psi}(z) \\
{\left[L_{n}^{(j)}, \psi(z)\right] } & =z^{n}\left(z \frac{d}{d z}+j(n+1)\right) \psi(z), \\
{\left[L_{n}^{(j)}, \bar{\psi}(z)\right] } & =z^{n}\left(z \frac{d}{d z}+(1-j)(n+1)\right) \bar{\psi}(z) .
\end{aligned}
$$


Thus the set $\left\{L_{n}^{(j)}\right\}_{n \in \mathbf{Z}}$ forms an infinite-dimensional Lie algebra called the Virasoro algebra with central charge $c=-2\left(6 j^{2}-6 j+1\right)$.

The field operators $\psi(z)$ and $\bar{\psi}(z)$, the current operator $J(z)$ and the energy-momentum tensor $T^{(j)}(z)$ form the so-called spin $j b c$-system or ghost system in the physics literature. For $j=1 / 2$ the $b c$-system is usually called abelian conformal field theory. In the present paper we shall discuss the $j=0$ ghost system and we shall also refer to this case as abelian conformal field theory.

\section{Universal Grassmann Manifold}

In this section we shall briefly recall the theory of the universal Grassmann manifold due to M. Sato $(\underline{\mathrm{SA}}])$. Let $\mathcal{W}^{\dagger}$ be the vector space introduced in the previous section.

Definition 2.1. The universal Grassmann manifold $\mathrm{UGM}^{p}$ of charge $p, p \in \mathbf{Z}$, is the set of closed subspace $U \subset \mathcal{W}^{\dagger}$ such that

1. The kernel and the cokernel of the natural linear map $f: U \rightarrow \mathcal{W}^{\dagger} / F^{0} \mathcal{W}^{\dagger}$ are of finite dimension;

2. $\operatorname{dim} \operatorname{Ker} f-\operatorname{dim}$ Coker $f=p$.

Put

$$
\mathrm{UGM}=\bigsqcup_{p \in \mathbf{Z}} \mathrm{UGM}^{p}
$$

Also, we can introduce the induced filtration $F^{m} U$ on $U$ by

$$
F^{m} U=U \cap F^{m} \mathcal{W}^{\dagger}
$$

Then we have

$$
\operatorname{dim} F^{m} U / F^{m+1} U \leq 1
$$

Put

$$
M(U)=\left\{m+1 / 2 \mid \operatorname{dim} F^{m} U / F^{m+1} U=1\right\} .
$$

It is easy to show that $M(U)$ is a Maya diagram of charge $p$.

Definition 2.2. For $U \in U G M^{p}$ a frame $\Xi$ of $U$ is a basis

$$
\Xi=\left\{\cdots, \zeta^{p-5 / 2}, \zeta^{p-3 / 2}, \zeta^{p-1 / 2}\right\}
$$

of $U$ such that there exists a half integer $\nu_{0}$ such that for any $\nu \leq \nu_{0}$

$$
\begin{aligned}
& \zeta^{\nu} \in F^{\nu-1 / 2} U \backslash F^{\nu+1 / 2} U \\
& \zeta^{\nu} \equiv e^{\nu} \quad\left(\bmod F^{\nu-1 / 2} U\right)
\end{aligned}
$$

For a frame

$$
\Xi=\left\{\cdots, \zeta^{p-5 / 2}, \zeta^{p-3 / 2}, \zeta^{p-1 / 2}\right\}
$$

of $U \in \mathrm{UGM}^{p}$ we can define the semi-infinite wedge product

$$
\cdots \wedge \zeta^{p-5 / 2} \wedge \zeta^{p-3 / 2} \wedge \zeta^{p-1 / 2}
$$


as an element of the fermion Fock space $\mathcal{F}^{\dagger}(p)$ by the following procedure. Put $n_{0}=$ $\nu_{0}-1 / 2$. For $n \leq n_{0}$ the element $\zeta^{n+1 / 2}$ can be written as

$$
\zeta^{n+1 / 2}=e^{n+1 / 2}+\sum_{k=n+1}^{\infty} a_{k+1 / 2}^{(n)} e^{k+1 / 2} .
$$

For $n \leq n_{0}$ we define the wedge product

$$
\cdots \wedge e^{n-3 / 2} \wedge e^{n-1 / 2} \wedge \zeta^{n+1 / 2} \wedge \cdots \wedge \zeta^{p-1 / 2}=\langle n| \wedge \zeta^{n+1 / 2} \wedge \cdots \wedge \zeta^{p-1 / 2}
$$

as the limit of the wedge products

$$
\langle n| \wedge \zeta_{m_{n+1}}^{n+1 / 2} \wedge \cdots \zeta_{m_{p}}^{p-1 / 2}
$$

where $\zeta_{m}^{\nu}$ is defined by

$$
\zeta_{m}^{\nu}=\sum_{k<m} b_{k+1 / 2}^{(\nu)} e^{k+1 / 2} \equiv \zeta^{\nu} \bmod F^{m} \mathcal{F}^{\dagger}
$$

Then the wedge product

$$
\langle n-1| \wedge \zeta^{n-1 / 2} \wedge \cdots \wedge \zeta^{p-1 / 2}
$$

contains all the terms appearing in

$$
\langle n| \wedge \zeta^{n+1 / 2} \wedge \cdots \wedge \zeta^{p-1 / 2} .
$$

Hence taking the limit $n \rightarrow-\infty$ we can define the semi-infinite product

$$
\cdots \wedge \zeta^{n-3 / 2} \wedge \zeta^{n-1 / 2} \wedge \zeta^{n+1 / 2} \wedge \cdots \wedge \zeta^{p-1 / 2} .
$$

If we use another frame $\Xi^{\prime}$ of $U$ the resulting wedge product is a non-zero constant multiple of the wedge product defined by the frame $\Xi$. Therefore, to each element $U \in$ $\mathrm{UGM}^{p}$ we can associate a one-dimensional subspace of $\mathcal{F}^{\dagger}(p)$, hence associated a point of the projective space $\mathbf{P}\left(\mathcal{F}^{\dagger}(p)\right)=\mathcal{F}^{\dagger}(p) \backslash\{0\} / \mathbf{C}^{*}$. We denote this 1-dimensional subspace of $\mathcal{F}^{\dagger}(p)$ by $\operatorname{det} U$.

Example 2.1. Let $\mathfrak{X}=(C ; Q ; \xi)$ be a one-pointed projective curve with formal coordinate, that is we assume that $Q$ is a smooth point of the curve $C$ and $\xi$ is a formal coordinate of the curve $C$ with center $Q$. Then to each meromorphic function $f \in H^{0}\left(C, \mathcal{O}_{C}(* Q)\right)$ we can associate its Laurent expansion $f(\xi)$ at $Q$ with respect to the coordinate $\xi$. This give an injective linear map from $H^{0}\left(C, \mathcal{O}_{C}(* Q)\right)$ to $\mathbf{C}((\xi))$. We identify $H^{0}\left(C, \mathcal{O}_{C}(* Q)\right)$ with the image and denote it by $U(\mathfrak{X})$. Identifying $\mathbf{C}((\xi))$ with $\mathcal{W}^{\dagger}$ as above, we can show that $U(\mathfrak{X})$ is closed subspace of $\mathbf{C}((\xi))$. Under this identification $\mathcal{W}^{\dagger} / F^{0} \mathcal{W}^{\dagger}$ becomes identified with $\mathbf{C}\left[\xi^{-1}\right] \xi^{-1}$. The natural map $f: U(\mathfrak{X}) \rightarrow \mathbf{C}\left[\xi^{-1}\right] \xi^{-1}$ is nothing but the map given by taking the principal part of the Laurent expansion of each meromorphic function. Hence Ker $f=H^{0}\left(C, \mathcal{O}_{C}\right)$. On the other hand by the exact sequence of sheaves

$$
0 \rightarrow \mathcal{O}_{C} \rightarrow \mathcal{O}_{C}(* Q) \rightarrow \mathbf{C}\left[\xi^{-1}\right] \xi^{-1} \rightarrow 0
$$

we obtain an exact sequence

$$
0 \rightarrow H^{0}\left(C, \mathcal{O}_{C}\right) \rightarrow H^{0}\left(C, \mathcal{O}_{C}(* Q)\right) \rightarrow \mathbf{C}\left[\xi^{-1}\right] \xi^{-1} \rightarrow H^{1}\left(C, \mathcal{O}_{C}\right) \rightarrow 0 .
$$


Therefore, we have

$$
\text { Coker } f \cong H^{1}\left(C, \mathcal{O}_{C}\right) \text {. }
$$

Put

$$
g=\operatorname{dim} H^{1}\left(C, \mathcal{O}_{C}\right),
$$

and call it the genus of the curve $C$. If the curve $C$ is non-singular this number $g$ is the usual genus of the curve $C$. Then, $U(\mathfrak{X})$ is a point of $\mathrm{UGM}^{1-g}$. A frame $\Xi$ is given by meromorphic functions $f_{j}$ whose Laurent expansion is of the form

$$
f_{1-g-j+1 / 2}=\xi^{-n_{j}}+\sum_{n=-n_{j}+1}^{\infty} a_{n}^{(j)} \xi^{n}, \quad j=1,2, \ldots
$$

where we may choose $f_{1-g-1 / 2}=1$, hence $n_{1}=0$. If our curve $C$ is non-singular or has only nodes, then by the Riemann-Roch theorem we have that

$$
n_{j}=j+g-1,
$$

for $j \geq g+1$. Put

$$
e\left(f_{1-g-j+1 / 2}\right)=e^{-n_{j}+1 / 2}+\sum_{n=-n_{j}+1}^{\infty} a_{n}^{(j)} e^{n+1 / 2} .
$$

For example we have $e\left(f_{1-g-1 / 2}\right)=e^{1 / 2}$. Then, the wedge product

$$
\cdots \wedge e\left(f_{1-g-5 / 2}\right) \wedge e\left(f_{1-g-3 / 2}\right) \wedge e\left(f_{1-g-1 / 2}\right)
$$

gives an element of $\mathcal{F}^{\dagger}(1-g)$ spanning $\operatorname{det} U(\mathfrak{X})$ and defines the point $\mathbf{P}\left(\mathcal{F}^{\dagger}(1-g)\right)$ associated to $U(\mathfrak{X})$. Since $\left.H^{0}\left(C, \mathcal{O}_{C}(* Q)\right)\right)$ is isomorphic to $U(\mathfrak{X})$ we may $\operatorname{define} \operatorname{det} H^{0}\left(C, \mathcal{O}_{C}(* Q)\right)=$ $\operatorname{det} U(\mathfrak{X})$ and so we can regard the wedge product (2.3) as an element of $\operatorname{det} H^{0}\left(C, \mathcal{O}_{C}(* Q)\right)$. Moreover, by (2.2) we get a natural isomorphism.

$$
\operatorname{det} H^{0}\left(C, \mathcal{O}_{C}\right) \otimes\left(\operatorname{det} H^{0}\left(C, \mathcal{O}_{C}(* Q)\right)\right)^{-1} \otimes \operatorname{det} \mathbf{C}\left[\xi^{-1}\right] \xi^{-1} \otimes\left(\operatorname{det} H^{1}\left(C, \mathcal{O}_{C}\right)\right)^{-1} \cong \mathbf{C} .
$$

Thus we may regard $\operatorname{det} H^{0}\left(C, \mathcal{O}_{C}(* Q)\right)$ as the determinant of the structure sheaf of the curve $C$, since there is a canonical isomorphism $\operatorname{det} \mathbf{C}\left[\xi^{-1}\right] \xi \cong \mathbf{C}$.

The map

$$
\Phi: \mathrm{UGM}^{p} \rightarrow \mathbf{P}\left(\mathcal{F}^{\dagger}(p)\right),
$$

which associates to each element $U \in \mathrm{UGM}^{p}$ the point $\operatorname{det} U \in \mathbf{P}\left(\mathcal{F}^{\dagger}(p)\right)$ is called the Plücker embedding. This is a generalization of the usual Plücker embedding for the usual Grassmann manifold. The following theorem is due to M. Sato and it plays an important role in soliton theory.

Theorem 2.1. The Plücker embedding $\Phi: \mathrm{UGM}^{p} \rightarrow \mathbf{P}\left(\mathcal{F}^{\dagger}(p)\right)$ is a holomorphic embedding. The image is a closed submanifold of $\mathbf{P}\left(\mathcal{F}^{\dagger}(p)\right)$ which is defined by Plücker's relations. 


\section{Ghost Vacua}

Now we shall develop the theory of the ghost system for the case $j=0$ in section 1 . In this case the Virasoro algebra have the following commutation relations

$$
\left[L_{n}^{(0)}, L_{m}^{(0)}\right]=(n-m) L_{n+m}^{(0)}-\frac{1}{6}\left(n^{3}-n\right) \delta_{m+n .0} .
$$

Also we have the following important commutation relations

$$
\begin{aligned}
& {\left[L_{n}^{(0)}, \psi(z)\right]=z^{n+1} \frac{d}{d z} \psi(z),} \\
& {\left[L_{n}^{(0)}, \bar{\psi}(z)\right]=z^{n}\left(z \frac{d}{d z}+(n+1)\right) \bar{\psi}(z) .}
\end{aligned}
$$

These commutation relations suggest that the field operator $\psi(z)$ behaves like a meromorphic function and $\bar{\psi}(z)$ behaves like a meromorphic one-form. This fact will be used to define the ghost vacua of the $j=0$ ghost system on a pointed curves with formal coordinates.

By an $N$-pointed curve with formal coordinates $\mathfrak{X}=\left(C ; Q_{1}, \ldots, Q_{N} ; \xi_{1}, \ldots, \xi_{N}\right)$ we mean that the curve $C$ is reduced and projective but not necessarily connected and that the points $Q_{j}$ are non-singular points of the curve $C$ and $\xi_{j}$ is a formal coordinate of the curve $C$ with center $Q_{j}$. See [U2] for further details regarding curves with formal coordinates. We will always assume that each of the connected components of $C$ contains at least one of the $Q_{j}^{\prime} s$. Put

$$
\begin{aligned}
\mathcal{F}_{N} & =\bigoplus_{p_{1}, \ldots, p_{N} \in \mathbf{Z}} \mathcal{F}\left(p_{1}\right) \otimes \cdots \otimes \mathcal{F}\left(p_{N}\right), \\
\mathcal{F}_{N}^{\dagger} & =\bigoplus_{p_{1}, \ldots, p_{N} \in \mathbf{Z}} \mathcal{F}^{\dagger}\left(p_{1}\right) \hat{\otimes} \cdots \hat{\otimes} \mathcal{F}^{\dagger}\left(p_{N}\right),
\end{aligned}
$$

where $\hat{\otimes}$ means the complete tensor product.

Definition 3.1. The ghost vacua $\mathcal{V}^{\dagger}{ }_{\mathrm{ab}}(\mathfrak{X})$ of the spin $j=0$ ghost system is the linear subspace of $\mathcal{F}_{N}^{\dagger}$ consisting of elements $\langle\Phi|$ satisfying the following conditions:

1. For all $|v\rangle \in \mathcal{F}_{N}$, there exists a meromorphic function $f \in H^{0}\left(C, \mathcal{O}_{C}\left(* \sum_{j=1}^{N} Q_{j}\right)\right)$ such that $\left\langle\Phi\left|\rho_{j}\left(\psi\left(\xi_{j}\right)\right)\right| v\right\rangle$ is the Laurent expansion of $f$ at the point $Q_{j}$ with respect to the formal coordinate $\xi_{j}$;

2. For all $|v\rangle \in \mathcal{F}_{N}$, there exists a meromorphic one-form $\omega \in H^{0}\left(C, \omega_{C}\left(* \sum_{j=1}^{N} Q_{j}\right)\right)$ such that $\left\langle\Phi\left|\rho_{j}\left(\bar{\psi}\left(\xi_{j}\right)\right)\right| v\right\rangle d \xi_{j}$ is the Laurent expansion of $\omega$ at the point $Q_{j}$ with respect to the coordinates $\xi_{j}$,

where $\rho_{j}(A)$ means that the operator $A$ acts on the $j$-th component of $\mathcal{F}_{N}$ as

$$
\rho_{j}(A)\left|u_{1} \otimes u_{2} \otimes \cdots \otimes u_{N}\right\rangle=(-1)^{p_{1}+\cdots+p_{j-1}}\left|u_{1} \otimes \cdots \otimes u_{j-1} \otimes A u_{j} \otimes u_{j+1} \otimes \cdots \otimes u_{N}\right\rangle .
$$


We will reformulate the above two conditions into gauge conditions. For that purpose we introduce the following notation.

For a meromorphic one-form $\omega \in H^{0}\left(C, \omega_{C}\left(* \sum_{j=1}^{N} Q_{j}\right)\right)$ we let

$$
\omega_{j}=\left(\sum_{n=-n_{0}}^{\infty} a_{n} \xi_{j}^{n}\right) d \xi_{j}
$$

be the Laurent expansion at $Q_{j}$ with respect to the coordinate $\xi_{j}$. Then, for the field operator $\psi(z)$ let us define $\psi\left[\omega_{j}\right]$ by

$$
\psi\left[\omega_{j}\right]=\operatorname{Res}_{\xi_{j}=0}\left(\psi\left(\xi_{j}\right) \omega_{j}\right)=\sum_{n=-n_{0}}^{\infty} a_{n} \psi_{n+1 / 2} .
$$

Similarly we can define $\bar{\psi}\left[\omega_{j}\right]$. For a meromorphic function $f \in H^{0}\left(C, \mathcal{O}_{C}\left(* \sum_{j=1}^{N} Q_{j}\right)\right)$ we let $f_{j}\left(\xi_{j}\right)$ be the Laurent expansion of $f$ at $Q_{j}$ with respect to the coordinate $\xi_{j}$. For the field operator $\psi(z)$ define $\psi\left[f_{j}\right]$ by

$$
\psi\left[f_{j}\right]=\underset{\xi_{j}=0}{\operatorname{Res}}\left(\psi\left(\xi_{j}\right) f_{j}\left(\xi_{j}\right) d \xi_{j}\right)
$$

Put

$$
\begin{aligned}
\psi[\omega]=\left(\psi\left[\omega_{1}\right], \ldots, \psi\left[\omega_{N}\right]\right), & \bar{\psi}[\omega] & =\left(\bar{\psi}\left[\omega_{1}\right], \ldots, \bar{\psi}\left[\omega_{N}\right]\right) \\
\psi[f]=\left(\psi\left[f_{1}\right], \ldots, \psi\left[f_{N}\right]\right), & \bar{\psi}[f] & =\left(\bar{\psi}\left[f_{1}\right], \ldots, \bar{\psi}\left[f_{N}\right]\right) .
\end{aligned}
$$

Then, these operate on $\mathcal{F}_{N}$ from the left and on $\mathcal{F}_{N}^{\dagger}$ from the right. For example, $\bar{\psi}[f]$ operates on $\mathcal{F}_{N}$ from the left by

$$
\begin{aligned}
\bar{\psi}[f]\left|u_{1} \otimes \cdots \otimes u_{N}\right\rangle & =\sum_{j=1}^{N} \rho_{j}\left(\bar{\psi}\left[f_{j}\right]\right)\left|u_{1} \otimes \cdots \otimes u_{N}\right\rangle \\
& =\sum_{j=1}^{N}(-1)^{p_{1}+\cdots+p_{j-1}\left|u_{1} \otimes \cdots \otimes u_{j-1} \otimes \bar{\psi}\left[f_{j}\right] u_{j} \otimes u_{j+1} \otimes \cdots \otimes u_{N}\right\rangle}
\end{aligned}
$$

for $\left|u_{j}\right\rangle \in \mathcal{F}^{\dagger}\left(p_{j}\right)$ and operates on $\mathcal{F}_{N}^{\dagger}$ from the right by

$$
\begin{aligned}
\left\langle v_{N} \otimes \cdots v_{1}\right| \bar{\psi}[f] & =\sum_{j=1}^{N}\left\langle v_{N} \otimes \cdots v_{1}\right| \rho_{j}\left(\bar{\psi}\left[f_{j}\right]\right) \\
& =\sum_{j=1}^{N}(-1)^{p_{1}+\cdots+p_{j-1}}\left\langle v_{N} \otimes \cdots \otimes v_{j+1} \otimes v_{j} \bar{\psi}\left[f_{j}\right] \otimes v_{j-1} \otimes \cdots \otimes v_{1}\right|
\end{aligned}
$$

for $\left\langle v_{j}\right| \in \mathcal{F}^{\dagger}\left(p_{j}\right)$.

Theorem 3.1. The element $\langle\Phi| \in \mathcal{F}_{N}^{\dagger}$ belongs to the space of ghost vacua $\mathcal{V}_{\mathrm{ab}}^{\dagger}(\mathfrak{X})$ of the $j=0$ ghost system if and only if $\langle\Phi|$ satisfies the following two conditions.

1. $\langle\Phi| \psi[\omega]=0$ for any meromorphic one-form $\omega \in H^{0}\left(C, \omega_{C}\left(* \sum_{j=1}^{N} Q_{j}\right)\right)$. 
2. $\langle\Phi| \bar{\psi}[f]=0$ for any meromorphic function $f \in H^{0}\left(C, \mathcal{O}_{C}\left(* \sum_{j=1}^{N} Q_{j}\right)\right)$.

The first (resp. second) condition in the above theorem is called the first (resp. second) gauge condition. The first and second gauge conditions can be rewritten in the following form:

1. $\sum_{j=1}^{N}(-1)^{p_{1}+\cdots+p_{j-1}}\left\langle\Phi \mid u_{1} \otimes \cdots \otimes \cdots \otimes u_{j-1} \otimes \psi\left[\omega_{j}\right] u_{j} \otimes u_{j+1} \otimes \cdots \otimes u_{N}\right\rangle=0$ for any $\omega \in H^{0}\left(C, \omega_{C}\left(* \sum_{j=1}^{N} Q_{j}\right)\right)$ and $\left|u_{j}\right\rangle \in \mathcal{F}\left(p_{j}\right), j=1,2, \ldots, N$.

2. $\sum_{j=1}^{N}(-1)^{p_{1}+\cdots+p_{j-1}}\left\langle\Phi \mid u_{1} \otimes \cdots \otimes u_{j-1} \otimes \bar{\psi}\left[f_{j}\right] u_{j} \otimes u_{j+1} \otimes \cdots \otimes u_{N}\right\rangle=0$ for any $f \in H^{0}\left(C, \mathcal{O}_{C}\left(* \sum_{j=1}^{N} Q_{j}\right)\right)$ and $\left|u_{j}\right\rangle \in \mathcal{F}\left(p_{j}\right), j=1,2, \ldots, N$.

It is easy to show that the ghost vacua $\mathcal{V}^{\dagger}$ ab $(\mathfrak{X})$ is a finite dimensional vector space. More strongly we can prove the following theorem.

Theorem 3.2. For any $N$-pointed curve $\mathfrak{X}=\left(C ; Q_{1}, \ldots, Q_{N} ; \xi_{1}, \ldots \xi_{N}\right)$ with formal coordinates we have

$$
\operatorname{dim}_{\mathbf{C}} \mathcal{V}^{\dagger}{ }_{\mathrm{ab}}(\mathfrak{X})=1 .
$$

A proof is given in section 5 .

In the later application we need to consider a disconnected curve. The following proposition is an immediate consequence of the definition.

Proposition 3.1. Let

$$
\mathfrak{X}_{1}=\left(C_{1} ; Q_{1}, \ldots, Q_{M} ; \xi_{1}, \ldots, \xi_{M}\right)
$$

and

$$
\mathfrak{X}_{2}=\left(C_{2} ; Q_{M+1}, \ldots, Q_{M} ; \xi_{M+1}, \ldots, \xi_{N}\right)
$$

be pointed curves with formal coordinates. Let $C$ be the disjoint union $C_{1} \sqcup C_{2}$ of the curves $C_{1}, C_{2}$. Put

$$
\mathfrak{X}=\left(C ; Q_{1}, \ldots, Q_{N} ; \xi_{1}, \ldots, \xi_{N}\right) \text {. }
$$

Then we have

$$
\mathcal{V}^{\dagger}{ }_{\mathrm{ab}}(\mathfrak{X})=\mathcal{V}_{\mathrm{ab}}^{\dagger}\left(\mathfrak{X}_{1}\right) \otimes \mathcal{V}^{\dagger}{ }_{\mathrm{ab}}\left(\mathfrak{X}_{2}\right) .
$$

Now we can introduce the dual ghost vacua.

Definition 3.2. Let $\mathcal{F}_{\mathrm{ab}}(\mathfrak{X})$ be the subspace of $\mathcal{F}_{N}$ spanned by $\psi[\omega] \mathcal{F}_{N}, \omega \in H^{0}\left(C, \omega_{C}\left(* \sum_{j=1}^{N} Q_{j}\right)\right)$ and $\bar{\psi}[f] \mathcal{F}_{N}, f \in H^{0}\left(C, \mathcal{O}\left(* \sum_{j=1}^{N} Q_{j}\right)\right)$. Put

$$
\mathcal{V}_{\mathrm{ab}}(\mathfrak{X})=\mathcal{F}_{N} / \mathcal{F}_{\mathrm{ab}}(\mathfrak{X}) .
$$

The quotients space $\mathcal{V}_{\mathrm{ab}}(\mathfrak{X})$ is called the space of dual ghost vacua of the $j=0$ ghost system.

Since $\mathcal{V}_{\mathrm{ab}}^{\dagger}(\mathfrak{X})$ is finite dimensional, $\mathcal{V}_{\mathrm{ab}}(\mathfrak{X})$ is dual to $\mathcal{V}_{\mathrm{ab}}^{\dagger}(\mathfrak{X})$. 
Example 3.1. Let us consider the one-dimensional complex projective space $\mathbf{P}^{1}=\mathbf{C} \cup$ $\{\infty\}$. Let $z$ be the coordinate of $\mathbf{C}$. We shall show that

$$
\operatorname{dim}_{\mathbf{C}} \mathcal{V}^{\dagger}{ }_{\mathrm{ab}}\left(\left(\mathbf{P}^{1} ; 0 ; z\right)\right)=1 \text {. }
$$

A basis of $H^{0}\left(\mathbf{P}^{1}, \omega_{\mathbf{P}^{1}}(* 0)\right)$ is given by

$$
\frac{d z}{z^{m+2}}, \quad m=0,1,2, \ldots
$$

A basis of $H^{0}\left(\mathbf{P}^{1}, \mathcal{O}_{\mathbf{P}^{1}}(* 0)\right)$ is given by

$$
\frac{1}{z^{m}}, \quad m=0,1,2, \ldots
$$

Then, we have that

$$
\psi\left[\frac{d z}{z^{m+2}}\right]=\psi_{-m-3 / 2}, \quad \bar{\psi}\left[\frac{1}{z^{m}}\right]=\bar{\psi}_{-m+1 / 2}, \quad m=0,1,2, \ldots
$$

Hence, an element $\langle\Phi| \in \mathcal{V}^{\dagger}{ }_{\mathrm{ab}}\left(\left(\mathbf{P}^{1} ; 0 ; z\right)\right)$ satisfies the equations

$$
\begin{aligned}
& \langle\Phi| \psi_{-m-3 / 2}=\langle\Phi| \wedge e^{-m-3 / 2}=0, \quad m=0,1,2, \ldots, \\
& \langle\Phi| \bar{\psi}_{-m+1 / 2}=\langle\Phi| i\left(e^{m-1 / 2}\right)=0, \quad m=0,1,2, \ldots .
\end{aligned}
$$

Note that any element of $\mathcal{F}$ is a finite linear combination of elements of the form

$$
\begin{aligned}
& \bar{\psi}_{\mu_{1}} \bar{\psi}_{\mu_{2}} \cdots \bar{\psi}_{\mu_{r}} \psi_{\nu_{s}} \psi_{\nu_{s-1}} \cdots \psi_{\nu_{1}}|0\rangle, \\
& \mu_{1}<\mu_{2}<\cdots<\mu_{r}<0, \quad \nu_{1}<\nu_{2}<\cdots<\nu_{s}<0, \quad r \geq 0, s \geq 0 .
\end{aligned}
$$

By the first gauge condition, if $r>0$ we have

$$
\left\langle\Phi\left|\bar{\psi}_{\mu_{1}} \bar{\psi}_{\mu_{2}} \cdots \bar{\psi}_{\mu_{r}} \psi_{\nu_{s}} \psi_{\nu_{s-1}} \cdots \psi_{\nu_{1}}\right| 0\right\rangle=0
$$

Moreover, by the second gauge condition if $s>0$ and $\nu_{s}<-1 / 2$ we have

$$
\left\langle\Phi\left|\psi_{\nu_{s}} \psi_{\nu_{s-1}} \cdots \psi_{\nu_{1}}\right| 0\right\rangle=0
$$

Thus we conclude that $\langle\Phi|$ is zero on all the element of the form (3.4) except $\bar{\psi}_{-1 / 2}|0\rangle=$ $|-1\rangle$. Therefore, $\langle\Phi|$ is a constant multiple of $\langle-1|$ and we conclude

$$
\mathcal{V}^{\dagger}{ }_{\mathrm{ab}}\left(\left(\mathbf{P}^{1} ; 0 ; z\right)\right)=\mathbf{C}\langle-1| \text {. }
$$

Let us assume that $C$ is a non-singular curve of genus $g \geq 1$. Let us consider a onepointed curve $\mathfrak{X}=(C ; Q ; \xi)$ with a formal coordinate. Choose a basis $\left\{\omega_{1}, \ldots, \omega_{g}\right\}$ of holomorphic one-forms on $C$. We let

$$
\omega_{i}=\left(\sum_{n=0}^{\infty} a_{n}^{(i)} \xi^{n}\right) d \xi, \quad i=1,2, \ldots, g
$$


be the Taylor expansions of $\omega_{i}$ 's at the point $Q$. For any positive integer $j$ choose a meromorphic one-form $\omega_{g+j} \in H^{0}\left(C, \omega_{C}(* Q)\right)$ in such a way that it has the Laurent expansion

$$
\omega_{g+j}=\left(\xi^{-(j+1)}+\sum_{n=-j}^{\infty} a_{n}^{(g+j)} \xi^{n}\right) d \xi
$$

at $Q$. Put

$$
\begin{aligned}
e\left(\omega_{i}\right) & =\sum_{n=0}^{\infty} a_{n}^{(i)} e^{n+1 / 2}, \\
e\left(\omega_{g+j}\right) & =e^{-j-1 / 2}+\sum_{n=-j}^{\infty} a_{n}^{(g+j)} e^{n+1 / 2} .
\end{aligned}
$$

Then, the infinite sums $e\left(\omega_{i}\right)$ and $e\left(\omega_{g+j}\right)$ are regarded as elements of $\mathcal{W}^{\dagger}$ (see section 2). Put

$$
\langle\omega(\mathfrak{X})|=\cdots \wedge e\left(\omega_{g+2}\right) \wedge e\left(\omega_{g+1}\right) \wedge e\left(\omega_{g}\right) \wedge \cdots \wedge e\left(\omega_{1}\right) .
$$

The set

$$
\left\{\ldots, \omega_{g+2}, \omega_{g+1}, \omega_{g}, \ldots, \omega_{1}\right\}
$$

is a frame of $H^{0}\left(C, \omega_{C}(* Q)\right) \subset \mathbf{C}((\xi)) d \xi$. Since $H^{0}\left(C, \omega_{C}(* Q)\right) \in \mathrm{UGM}^{g-1}$, we see that $\langle\omega(\mathfrak{X})| \in \mathcal{F}^{\dagger}(g-1)$ is non-zero. By a similar arguments as in Example 2.1 we may also regard $\langle\omega(\mathfrak{X})|$ as an element of the determinant of the canonical sheaf $\omega_{C}$. Note of course that $\langle\omega(\mathfrak{X})|$ depends on the choice of the basis $\left(\omega_{i}\right)$.

Lemma 3.1. The semi-infinite wedge product $\langle\omega(\mathfrak{X})|$ is a non-zero element of $\mathcal{F}^{\dagger}(g-1)$ which satisfies the first and second gauge conditions. Hence

$$
\langle\omega(\mathfrak{X})| \in \mathcal{V}^{\dagger}{ }_{\mathrm{ab}}(\mathfrak{X}) .
$$

Proof. For any element $\omega \in H^{0}\left(C, \omega_{C}(* Q)\right)$ we let $\left(\sum_{n=-n_{0}}^{\infty} a_{n} \xi^{n}\right) d \xi$ be its Laurent expansion. Then we have

$$
\psi[\omega]=\sum_{n=-n_{0}}^{\infty} a_{n} \psi_{n+1 / 2}
$$

whose right action on $\mathcal{F}^{\dagger}$ is given by

$$
\wedge\left(\sum_{n=-n_{0}}^{\infty} a_{n} e^{n+1 / 2}\right)=\wedge e(\omega) .
$$

Hence we have that

$$
\langle\omega(\mathfrak{X})| \wedge e(\omega)=0 .
$$

Thus $\langle\omega(\mathfrak{X})|$ satisfies the first gauge condition.

Now let $\sum_{m=-m_{0}}^{\infty} b_{m} \xi^{m}$ be the Laurent expansion of a meromorphic function $f \in$ $H^{0}\left(C, \mathcal{O}_{C}(* Q)\right)$ at $Q$. Then we have

$$
\bar{\psi}[f]=\sum_{m=-m_{0}}^{\infty} b_{m} \psi_{m+1 / 2}
$$


and its right action is given by

$$
\sum_{m=-m_{0}}^{\infty} b_{m} i\left(e^{-m-1 / 2}\right)
$$

Then we have that

$$
\begin{aligned}
e(\omega) \bar{\psi}[f] & =\left(\sum_{n=-n_{0}} a_{n} e^{n+1 / 2}\right)\left(\sum_{m=-m_{0}}^{\infty} b_{m} i\left(e^{-m-1 / 2}\right)\right) \\
& =\sum_{m=-m_{0}}^{\infty} a_{-m-1} b_{m} \\
& =\operatorname{Res}_{\xi=0}(f(\xi) \omega)=0 .
\end{aligned}
$$

Thus the second gauge condition is also satisfied.

Corollary 3.1. Let $1=l_{1}<l_{2}<\cdots<l_{g} \leq 2 g-1$ be the Weierstrass gap values of the curve $C$ at the point $Q$. Then,

$\bar{\psi}\left[\xi^{-l_{g}}\right] \bar{\psi}\left[\xi^{-l_{g-1}}\right] \cdots \bar{\psi}\left[\xi^{-l_{1}}\right]|-1\rangle=\bar{e}_{l_{g}-1 / 2} \wedge \bar{e}_{l_{g-1}-1 / 2} \wedge \cdots \wedge \bar{e}_{l_{1}-1 / 2} \wedge \bar{e}_{-3 / 2} \wedge \bar{e}_{-5 / 2} \wedge \cdots$

defines a non-zero element of $\mathcal{V}_{\mathrm{ab}}(\mathfrak{X})=\mathcal{F} / \mathcal{F}(\mathfrak{X})$.

Proof. The Weierstrass gap values have the property (for details see Lemma 6.2):

$$
\left\langle e\left(\omega_{g}\right) \wedge \cdots \wedge e\left(\omega_{1}\right) \mid \bar{e}_{l_{g}-1 / 2} \wedge \bar{e}_{l_{g-1}-1 / 2} \wedge \cdots \wedge \bar{e}_{l_{1}-1 / 2}\right\rangle \neq 0 .
$$

On the other hand in the infinite wedge product

$$
\cdots \wedge e\left(\omega_{g+n}\right) \wedge \cdots \wedge e\left(\omega_{g+2}\right) \wedge e\left(\omega_{g+1}\right)
$$

the term $\langle-1|$ only appears when we choose the term $e^{-j-1 / 2}$ of $e\left(\omega_{g+j}\right)$ for each $j \geq$ 1. Other terms do not have a term $e^{-m-1 / 2}$ for a certain positive integer $m$. Since $\left\langle e\left(\omega_{g}\right) \wedge \cdots \wedge e\left(\omega_{1}\right)\right|$ does not contain a term $e^{-m-1 / 2}$ for any $m \geq 1$ we have that

$\left\langle\omega(\mathfrak{X})\left|\bar{\psi}\left[\xi^{-l_{g}}\right] \bar{\psi}\left[\xi^{-l_{g-1}}\right] \cdots \bar{\psi}\left[\xi^{-l_{1}}\right]\right|-1\right\rangle=\left\langle e\left(\omega_{g}\right) \wedge \cdots \wedge e\left(\omega_{1}\right) \mid \bar{e}_{l_{g}-1 / 2} \wedge \bar{e}_{l_{g-1}-1 / 2} \wedge \cdots \wedge \bar{e}_{l_{1}-1 / 2}\right\rangle \neq 0$.

QED

Theorem 3.3. The space of ghost vacua $\mathcal{V}^{\dagger}$ ab $(\mathfrak{X})$ is isomorphic to the determinant of the canonical bundle $\omega_{C}$.

Notice that this theorem follows directly from Lemma 3.1, Theorem 3.2 and the discussion in section 2. The isomorphism of course depends on the choice of a basis for $H^{0}\left(C, \omega_{C}(* Q)\right)$.

Let $\mathfrak{X}=\left(C ; Q_{1}, \ldots, Q_{N} ; \xi_{1}, \ldots, \xi_{N}\right)$ be an $N$-pointed curve with formal coordinates. Let $Q_{N+1}$ be a non-singular point and choose a formal coordinate $\xi_{N+1}$ of $C$ with center $Q_{N+1}$. Put

$$
\widetilde{\mathfrak{X}}=\left(C ; Q_{1}, \ldots, Q_{N}, Q_{N+1} ; \xi_{1}, \ldots, \xi_{N}, \xi_{N+1}\right) .
$$


Then the canonical linear mapping

$$
\begin{aligned}
\iota: \mathcal{F}_{N} & \rightarrow \mathcal{F}_{N+1} \\
|v\rangle & \mapsto|v\rangle \otimes|0\rangle
\end{aligned}
$$

induces the canonical mapping

$$
\iota^{*}: \mathcal{F}_{N+1}^{\dagger} \rightarrow \mathcal{F}_{N}^{\dagger}
$$

Theorem 3.4. The canonical mapping $\iota^{*}$ induces an isomorphism

$$
\mathcal{V}^{\dagger}{ }_{\mathrm{ab}}(\widetilde{\mathfrak{X}}) \cong \mathcal{V}_{\mathrm{ab}}^{\dagger}(\mathfrak{X}) \text {. }
$$

This isomorphism is denoted the "Propagation of vacua" isomorphism.

Proof. Since we have injective maps

$$
\begin{aligned}
H^{0}\left(C, \omega_{C}\left(* \sum_{j=1}^{N} Q_{j}\right)\right) & \hookrightarrow H^{0}\left(C, \omega_{C}\left(* \sum_{j=1}^{N+1} Q_{j}\right)\right), \\
H^{0}\left(C, \mathcal{O}_{C}\left(* \sum_{j=1}^{N} Q_{j}\right)\right) & \hookrightarrow H^{0}\left(C, \mathcal{O}_{C}\left(* \sum_{j=1}^{N+1} Q_{j}\right)\right),
\end{aligned}
$$

and for $f(\xi) \in \mathbf{C}[[\xi]]$ we have

$$
\psi[f(\xi) d \xi]|0\rangle=0, \quad \bar{\psi}[f(\xi)|0\rangle=0,
$$

the image $\iota^{*}\left(\mathcal{V}^{\dagger}{ }_{\mathrm{ab}}(\hat{\mathfrak{X}})\right)$ is contained in $\mathcal{V}^{\dagger}{ }_{\mathrm{ab}}(\mathfrak{X})$.

Therefore, it is enough to show that any element $\langle\phi| \in \mathcal{V}^{\dagger}{ }_{\mathrm{ab}}(\mathfrak{X})$ uniquely determines an element $\langle\Phi| \in \mathcal{V}^{\dagger}{ }_{\text {ab }}(\hat{\mathfrak{X}})$ such that $\iota^{*}(\langle\Phi|)=\langle\phi|$. Note that any element of $\mathcal{F}$ is a finite linear combination of elements of the form

$$
\begin{aligned}
& \bar{\psi}_{\mu_{1}} \bar{\psi}_{\mu_{2}} \cdots \bar{\psi}_{\mu_{r}} \psi_{\nu_{s}} \psi_{\nu_{s-1}} \cdots \psi_{\nu_{1}}|0\rangle, \\
& \mu_{1}<\mu_{2}<\cdots<\mu_{r}<0, \quad \nu_{1}<\nu_{2}<\cdots<\nu_{s}<0, \quad r \geq 0, s \geq 0 .
\end{aligned}
$$

By a double induction on $r$ and $s$ we shall show that $\langle\phi|$ uniquely determines the value

$$
\left\langle\Phi\left|\bar{\psi}_{\mu_{1}} \bar{\psi}_{\mu_{2}} \cdots \bar{\psi}_{\mu_{r}} \psi_{\nu_{s}} \psi_{\nu_{s-1}} \cdots \psi_{\nu_{1}}\right| 0\right\rangle
$$

in such a way that $\langle\Phi|$ satisfies the first and second gauge conditions.

For $(r, s)=(0,0)$ put

$$
\langle\Phi \mid u \otimes 0\rangle=\langle\phi \mid u\rangle
$$

for any $|u\rangle \in \mathcal{F}_{N}$.

Choose $\left|u_{j}\right\rangle \in \mathcal{F}\left(p_{j}\right)$ and put

$$
|u\rangle=\left|u_{1}\right\rangle \otimes \cdots \otimes\left|u_{N}\right\rangle .
$$

Choose $\omega \in H^{0}\left(C, \omega_{C}\left(* \sum_{j=1}^{N} Q_{j}+(n+1) Q_{N+1}\right)\right)$ in such a way that its Laurent expansion at the point $Q_{N+1}$ has the form

$$
\omega_{N+1}=\left(\xi_{N+1}^{-n-1}+\sum_{n=0}^{\infty} a_{n} \xi_{N+1}^{n}\right) d \xi
$$


Then we have

$$
\psi\left[\omega_{N+1}\right]|0\rangle=\psi_{-n-1 / 2}|0\rangle=i\left(\bar{e}_{-n-1 / 2}\right)|0\rangle .
$$

This is no-zero if and only if $n$ is a non-negative integer. For a non-negative integer $n$ define

$$
\left\langle\Phi\left|u \otimes \psi_{-n-1 / 2}\right| 0\right\rangle=(-1)^{p_{1}+\cdots p_{N}+1} \sum_{j=1}^{N}\left\langle\Phi \mid \rho_{j}\left(\psi\left[\omega_{j}\right]\right) u \otimes 0\right\rangle .
$$

This is independent of the choice of $\omega$ satisfying (3.6) , since if $\omega^{\prime} \in H^{0}\left(C, \omega_{C}\left(* \sum_{j=1}^{N} Q_{j}+\right.\right.$ $\left.\left.(n+1) Q_{N+1}\right)\right)$ satisfies (3.6) $\omega-\omega^{\prime}$ is holomorphic at $Q_{N+1}$. Thus $\left\langle\Phi\left|u \otimes \psi_{-n-1 / 2}\right| 0\right\rangle$ is well-defined for any integer $n$. Then for any meromorphic form $\tau \in H^{0}\left(C, \omega\left(* \sum_{n=1}^{N+1} Q_{j}\right)\right)$ we have

$$
\sum_{j=1}^{N+1}\left\langle\Phi \mid \rho_{j}\left(\psi\left[\tau_{j}\right]\right) u \otimes 0\right\rangle=0 .
$$

This establishes the first gauge condition on this subspace.

Next let us define

$$
\left\langle\Phi\left|u \otimes \psi_{-n_{2}-1 / 2} \psi_{-n_{1}-1 / 2}\right| 0\right\rangle
$$

with $0 \leq n_{2}<n_{1}$. Choose a meromorphic form $\widetilde{\omega} \in H^{0}\left(C, \omega_{C}\left(* \sum_{j=1}^{N} Q_{j}+\left(n_{2}+1\right) Q_{N+1}\right)\right)$ which has the Laurent expansion

$$
\widetilde{\omega}_{N+1}=\left(\xi_{N+1}^{-n_{2}-1}+\sum_{n=0}^{\infty} b_{n} \xi_{N+1}^{n}\right) d \xi
$$

Define

$$
\left\langle\Phi\left|u \otimes \psi_{-n_{2}-1 / 2} \psi_{-n_{1}-1 / 2}\right| 0\right\rangle=(-1)^{p_{1}+\cdots p_{N}+1} \sum_{j=1}^{N}\left\langle\Phi \mid \rho_{j}\left(\psi\left[\widetilde{\omega}_{j}\right]\right) u \otimes \psi_{-n_{1}-1 / 2} 0\right\rangle .
$$

We need to show that this is well-defined. Choose another $\widetilde{\omega}^{\prime}$ which has the Laurent expansion of the same type (3.9). Then $\tau=\widetilde{\omega}-\widetilde{\omega}^{\prime}$ is holomorphic at $Q_{N+1}$, hence

$$
\psi\left[\tau_{N+1}\right] \psi_{-n_{1}-1 / 2}|0\rangle=0 .
$$

Note that if $j>k$ we have that

$$
\begin{aligned}
\rho_{j}\left(\psi\left[\omega_{j}\right]\right) \rho_{k}\left(\psi\left[\tau_{k}\right]\right)|u\rangle \\
\quad=(-1)^{p_{1}+\cdots+p_{k-1}} \rho_{j}\left(\psi\left[\omega_{j}\right]\right)\left|u_{1} \otimes \cdots \otimes \psi\left[\tau_{k}\right] u_{k} \otimes \cdots u_{N}\right\rangle \\
\quad=(-1)^{\left(p_{1}+\cdots+p_{k-1}\right)+\left(p_{1}+\cdots+p_{k-1}+\cdots+p_{j-1}\left|u_{1} \otimes \cdots \otimes \psi\left[\tau_{k}\right] u_{k} \otimes \cdots \otimes \psi\left[\omega_{j}\right] u_{j} \otimes \cdots u_{N}\right\rangle\right.} \\
\quad=-\rho_{k}\left(\psi\left[\tau_{k}\right]\right) \rho_{j}\left(\psi\left[\omega_{j}\right]\right)|u\rangle .
\end{aligned}
$$


We have the same result in the case $j \leq k$. Hence by (3.7) we have that

$$
\begin{aligned}
\sum_{j=1}^{N} & \left\langle\Phi \mid \rho_{j}\left(\psi\left[\tau_{j}\right]\right) u \otimes \psi_{-n_{1}-1 / 2} 0\right\rangle \\
& =(-1)^{p_{1}+\cdots p_{N}+1} \sum_{k=1}^{N} \sum_{j=1}^{N}\left\langle\Phi \mid \rho_{k}\left(\psi\left[\omega_{k}\right]\right) \rho_{j}\left(\psi\left[\tau_{j}\right]\right) u \otimes 0\right\rangle \\
& =(-1)^{p_{1}+\cdots p_{N}} \sum_{k=1}^{N}\left\{\sum_{j=1}^{N}\left\langle\Phi \mid \rho_{j}\left(\psi\left[\tau_{j}\right]\right) \rho_{k}\left(\psi\left[\omega_{k}\right]\right) u \otimes 0\right\rangle\right\} \\
& =(-1)^{p_{1}+\cdots p_{N}} \sum_{k=1}^{N}\left\{\sum_{j=1}^{N+1}\left\langle\Phi \mid \rho_{j}\left(\psi\left[\tau_{j}\right]\right) \rho_{k}\left(\psi\left[\omega_{k}\right]\right) u \otimes 0\right\rangle\right\} \\
& =0,
\end{aligned}
$$

where the last equality is a consequence of (3.8). Now by the same argument as above we can show that for any $\tau \in H^{0}\left(C, \omega_{C}\left(* \sum_{j=1}^{N+1}\right)\right)$ and any non-negative integer $n$ the first gauge condition holds on the subspace spanned by $\left|u \otimes \psi_{-n-1 / 2} 0\right\rangle$ :

$$
\sum_{j=1}^{N+1}\left\langle\Phi \mid \rho_{j}\left(\psi\left[\tau_{j}\right]\right) u \otimes \psi_{-n-1 / 2} 0\right\rangle=0 .
$$

In this way, by induction on $r$ we can show that for any non-positive integers $n_{1}>n_{2}>$ $\cdots>n_{r} \geq 0$

$$
\left\langle\Phi \mid u \otimes \psi_{-n_{1}-1 / 2} \cdots \psi_{-n_{r}-1 / 2} 0\right\rangle
$$

is well-defined and we have the first gauge condition on the subspace spanned by $\mid u \otimes$ $\left.\psi_{-n_{1}-1 / 2} \cdots \psi_{-n_{r}-1 / 2} 0\right\rangle$ :

$$
\sum_{j=1}^{N+1}\left\langle\Phi \mid \rho_{j}\left(\psi\left[\tau_{j}\right]\right) u \otimes \psi_{-n_{1}-1 / 2} \cdots \psi_{-n_{r-1}-1 / 2} 0\right\rangle=0 .
$$

Next let us define

$$
\left\langle\Phi \mid u \otimes \bar{\psi}_{-n-1 / 2} \psi_{-n_{1}-1 / 2} \cdots \psi_{-n_{r}-1 / 2} 0\right\rangle .
$$

Choose $f \in H^{0}\left(C, \mathcal{O}_{C}\left(* \sum_{j=1}^{N} Q_{j}+(n+1) Q_{N+1}\right)\right)$ in such a way that it has the Laurent expansion

$$
f_{N+1}=\xi_{N+1}^{-n-1}+\sum_{n=0}^{\infty} a_{n} \xi_{N+1}^{n}
$$

at the point $Q_{N+1}$. Then we have

$$
\begin{aligned}
\bar{\psi}\left[f_{N+1}\right] \psi_{-n_{1}-1 / 2} \cdots \psi_{-n_{r-1}-1 / 2}|0\rangle & =\bar{\psi}_{-n-1 / 2} \psi_{-n_{1}-1 / 2} \cdots \psi_{-n_{r-1}-1 / 2}|0\rangle \\
& \left.=\bar{e}_{n+1 / 2} \wedge\left|\psi_{-n_{1}-1 / 2} \cdots \psi_{-n_{r-1}-1 / 2}\right| 0\right\rangle
\end{aligned}
$$


This is no-zero if $n$ is a non-negative integer. For non-negative integer $n$ define

$$
\begin{aligned}
\langle\Phi| u & \left.\otimes \bar{\psi}_{-n-1 / 2} \psi_{-n_{1}-1 / 2} \cdots \psi_{-n_{r-1}-1 / 2} 0\right\rangle \\
& =(-1)^{p_{1}+\cdots p_{N}+1} \sum_{j=1}^{N}\left\langle\Phi\left|\rho_{j}\left(\bar{\psi}\left[f_{j}\right]\right) u \otimes \psi_{-n_{1}-1 / 2} \cdots \psi_{-n_{r-1}-1 / 2}\right| 0\right\rangle .
\end{aligned}
$$

This is independent of the choice of $f$ satisfying (3.12), since if $f^{\prime}$ satisfies (3.12) $f-f^{\prime}$ is holomorphic at $Q_{N+1}$. Thus

$$
\left\langle\Phi\left|u \otimes \bar{\psi}_{-n-1 / 2} \psi_{-n_{1}-1 / 2} \cdots \psi_{-n_{r-1}-1 / 2}\right| 0\right\rangle
$$

is well-defined for any integer $n$. Then for any meromorphic function $h \in H^{0}\left(C, \mathcal{O}\left(* \sum_{n=1}^{N+1} Q_{j}\right)\right)$ we have that

$$
\sum_{j=1}^{N+1}\left\langle\Phi\left|\rho_{j}\left(\bar{\psi}\left[h_{j}\right]\right) u \otimes \psi_{-n_{1}-1 / 2} \cdots \psi_{-n_{r-1}-1 / 2}\right| 0\right\rangle=0,
$$

establishing the second gauge condition on this subspace.

Now by induction on $s$ we can show that

$$
\left\langle\Phi\left|u \otimes \bar{\psi}_{-n_{s}-1 / 2} \cdots \bar{\psi}_{-n_{1}-1 / 2} \psi_{-n_{1}-1 / 2} \cdots \psi_{-n_{r-1}-1 / 2}\right| 0\right\rangle
$$

are well-defined and they satisfy the second gauge condition:

$$
\sum_{j=1}^{N+1}\left\langle\Phi\left|\rho_{j}\left(\bar{\psi}\left[f_{j}\right]\right) u \otimes \bar{\psi}_{-n_{s-1}-1 / 2} \cdots \bar{\psi}_{-n_{1}-1 / 2} \psi_{-n_{1}-1 / 2} \cdots \psi_{-n_{r-1}-1 / 2}\right| 0\right\rangle=0 .
$$

Thus $\langle\Phi| \in \mathcal{V}^{\dagger}{ }_{\mathrm{ab}}(\widetilde{\mathfrak{X}})$ has been uniquely constructed from $\langle\phi| \in \mathcal{V}^{\dagger}{ }_{\mathrm{ab}}(\mathfrak{X})$. By our construction we have that

$$
\iota^{*}(\langle\Phi|)=\langle\phi| .
$$

Next let us consider a curve $C$ with a node $P$. Let $\widetilde{C}$ be the curve obtained by resolving the singularity at $P$ and let $\pi: \widetilde{C} \rightarrow C$ be the natural holomorphic mapping. Then $\pi^{-1}(P)$ consists of two points $P_{+}$and $P_{-}$. Let

$$
\mathfrak{X}=\left(C ; Q_{1}, \ldots, Q_{N} ; \xi_{1}, \ldots, \xi_{N}\right)
$$

be an $N$-pointed curve with formal coordinates and we let

$$
\widetilde{\mathfrak{X}}=\left(\widetilde{C} ; P_{+}, P_{-}, Q_{1}, \ldots, Q_{N} ; z, w, \xi_{1}, \ldots, \xi_{N}\right)
$$

be the associated $N+2$-pointed curve with formal coordinates. Define an element $\left|0_{+,-}\right\rangle \in$ $\mathcal{F} \otimes \mathcal{F}$ by

$$
\left|0_{+,-}\right\rangle=|0\rangle \otimes|-1\rangle-|-1\rangle \otimes|0\rangle .
$$

The natural inclusion

$$
\begin{aligned}
\mathcal{F}_{N} & \hookrightarrow \mathcal{F}_{N+2} \\
|u\rangle & \mapsto\left|0_{+,-}\right\rangle \otimes|u\rangle
\end{aligned}
$$

defines a natural linear mapping

$$
\iota_{+,-}^{*}: \mathcal{F}_{N+2}^{\dagger} \rightarrow \mathcal{F}_{N}^{\dagger} \text {. }
$$


Theorem 3.5. The natural mapping $\iota_{+,-}^{*}$ induces a natural isomorphism

$$
\mathcal{V}^{\dagger}{ }_{\mathrm{ab}}(\widetilde{\mathfrak{X}}) \cong \mathcal{V}^{\dagger}{ }_{\mathrm{ab}}(\mathfrak{X}) \text {. }
$$

Proof. First let us show that

$$
\iota_{+,-}^{*}\left(\mathcal{V}^{\dagger}{ }_{\mathrm{ab}}(\widetilde{\mathfrak{X}})\right) \subset \mathcal{V}^{\dagger}{ }_{\mathrm{ab}}(\mathfrak{X}) .
$$

For $\langle\widetilde{\Phi}| \in \mathcal{V}^{\dagger}{ }_{\text {ab }}(\widetilde{\mathfrak{X}})$ put $\langle\Phi|=\iota_{+,-}^{*}(\langle\widetilde{\Phi}|)$. For $|u\rangle \in \mathcal{F}_{N}$ we have that

$$
\langle\Phi \mid u\rangle=\left\langle\widetilde{\Phi} \mid 0_{+,-} \otimes u\right\rangle .
$$

For a meromorphic one-form $\omega \in H^{0}\left(C, \omega_{C}\left(* \sum_{j=1}^{N} Q_{j}\right)\right)$ put $\widetilde{\omega}=\pi^{*}(\omega)$. Then $\widetilde{\omega}$ has a Laurent expansions at $P_{+}$and $P_{-}$of the following form:

$$
\begin{aligned}
& \widetilde{\omega}_{+}=\left(\frac{a_{-1}}{z}+a_{0}+a_{1} z+a_{2} z^{2}+\cdots\right) d z \\
& \widetilde{\omega}_{-}=\left(-\frac{a_{-1}}{w}+b_{0}+b_{1} w+b_{2} w^{2}+\cdots\right) d w
\end{aligned}
$$

Then we have that

$$
\begin{aligned}
\rho_{+}\left(\psi\left[\widetilde{\omega}_{+}\right]\right)\left|0_{+,-}\right\rangle & =\psi\left[\widetilde{\omega}_{+}\right]|0\rangle \otimes|-1\rangle-\psi\left[\widetilde{\omega}_{+}\right]|-1\rangle \otimes|0\rangle \\
& =a_{-1}|-1\rangle \otimes|-1\rangle
\end{aligned}
$$

Similarly we have that

$$
\begin{aligned}
\rho_{-}\left(\psi\left[\widetilde{\omega}_{-}\right]\right)\left|0_{+,-}\right\rangle & =|0\rangle \otimes \psi\left[\widetilde{\omega}_{-}\right]|-1\rangle+|-1\rangle \otimes \psi\left[\widetilde{\omega}_{-}\right]|0\rangle \\
& =-a_{-1}|-1\rangle \otimes|-1\rangle .
\end{aligned}
$$

Since $\omega$ and $\widetilde{\omega}$ have the same Laurent expansions at $Q_{j}$, by the above results we have that

$$
\begin{aligned}
& \sum_{j=1}^{N}\left\langle\Phi \mid \rho_{j}\left(\psi\left[\widetilde{\omega}_{j}\right]\right) u\right\rangle \\
& =\sum_{j=1}^{N}\left\langle\widetilde{\Phi} \mid 0_{+,-} \otimes \rho_{j}\left(\psi\left[\widetilde{\omega}_{j}\right]\right) u\right\rangle \\
& =\left\langle\widetilde{\Phi} \mid \rho_{+}\left(\psi\left[\widetilde{\omega}_{+}\right]\right) 0_{+,-} \otimes u\right\rangle+\left\langle\widetilde{\Phi} \mid \rho_{-}\left(\psi\left[\widetilde{\omega}_{+}\right]\right) 0_{+,-} \otimes u\right\rangle+\sum_{j=1}^{N}\left\langle\widetilde{\Phi} \mid 0_{+,-} \otimes \rho_{j}\left(\psi\left[\widetilde{\omega}_{j}\right]\right) u\right\rangle \\
& =0
\end{aligned}
$$

by the first gauge condition for $\langle\widetilde{\Phi}|$. Thus $\langle\Phi|$ satisfies the first gauge condition. Similarly we can show that $\langle\Phi|$ satisfies the second gauge condition. Hence $\langle\Phi| \in \mathcal{V}^{\dagger}{ }_{\text {ab }}(\mathfrak{X})$.

Now let us show that $\iota_{+,-}^{*}$ is bijective. For that purpose it is enough to show that $\langle\Phi| \in \mathcal{V}^{\dagger}{ }_{\mathrm{ab}}(\mathfrak{X})$ determines uniquely $\langle\widetilde{\Phi}| \in \mathcal{V}_{\mathrm{ab}}^{\dagger}(\widetilde{\mathfrak{X}})$. Choose $f \in H^{0}\left(\widetilde{C}, \mathcal{O}_{\widetilde{C}}\left(* \sum_{j=1}^{N} Q_{j}\right)\right)$ such that the Taylor expansions at $P_{ \pm}$have the forms

$$
\begin{aligned}
& f_{+}=-1+\sum_{n=1}^{\infty} a_{n} z^{n}, \\
& f_{-}=\sum_{n=1}^{\infty} b_{n} w^{n} .
\end{aligned}
$$


Then we have that

$$
\begin{aligned}
& \bar{\psi}\left[f_{+}\right]=-\bar{\psi}_{1 / 2}+\sum_{n=1}^{\infty} a_{n} \bar{\psi}_{n+1 / 2} \\
& \bar{\psi}\left[f_{-}\right]=\sum_{n=1}^{\infty} b_{n} \bar{\psi}_{n+1 / 2} .
\end{aligned}
$$

Hence we have that

$$
\begin{aligned}
\rho_{+}\left(\bar{\psi}\left[f_{+}\right]\right)\left|0_{+,-}\right\rangle & =\bar{\psi}\left[f_{+}\right]|0\rangle \otimes|-1\rangle-\bar{\psi}\left[f_{+}\right]|-1\rangle \otimes|0\rangle=|0\rangle \otimes|0\rangle, \\
\rho_{-}\left(\bar{\psi}\left[f_{-}\right]\right)\left|0_{+,-}\right\rangle & =|0\rangle \otimes \bar{\psi}\left[f_{-}\right]|-1\rangle+|-1\rangle \otimes \bar{\psi}\left[f_{-}\right]|0\rangle=0 .
\end{aligned}
$$

Now define

$$
\langle\widetilde{\Phi} \mid 0 \otimes 0 \otimes u\rangle=\sum_{j=1}^{N}\left\langle\widetilde{\Phi} \mid 0_{+,-} \otimes \rho_{j}\left(\bar{\psi}\left[f_{j}\right]\right) u\right\rangle=\sum_{j=1}^{N}\left\langle\Phi \mid \rho_{j}\left(\bar{\psi}\left[f_{j}\right]\right) u\right\rangle .
$$

This is well-defined, since if $f^{\prime} \in H^{0}\left(\widetilde{C}, \mathcal{O}_{\widetilde{C}}\left(* \sum_{j=1}^{N} Q_{j}\right)\right)$ has the same type of Taylor expansions (3.16) and (3.17), then $h=f-f^{\prime}$ is an element of $H^{0}\left(C, \mathcal{O}_{C}\left(* \sum_{j=1}^{N} Q_{j}\right)\right)$. Hence

$$
\sum_{j=1}^{N}\left\langle\Phi \mid \rho_{j}\left(\bar{\psi}\left[h_{j}\right]\right) u\right\rangle=0
$$

Now let us show that $\langle\widetilde{\Phi}|$ just defined satisfies the first gauge condition for any element $h \in$ $H^{0}\left(\widetilde{C}, \mathcal{O}_{\widetilde{C}}\left(* \sum_{j=1}^{N} Q_{j}\right)\right)$ and the second gauge condition for any $\omega \in H^{0}\left(\widetilde{C}, \omega_{\widetilde{C}}\left(* \sum_{j=1}^{N} Q_{j}\right)\right)$ applied to the vectors $|0 \otimes 0 \otimes u\rangle$. Let

$$
\begin{aligned}
& \omega_{+}=\left(a_{0}+a_{1} z+\cdots\right) d z \\
& \omega_{-}=\left(b_{0}+b_{1} w+\cdots\right) d w
\end{aligned}
$$

be the Taylor expansions of $\omega$ at $P_{ \pm}$. Then we have that

$$
\psi\left[\omega_{+}\right]|0\rangle \otimes|0\rangle=0, \quad \psi\left[\omega_{-}\right]|0\rangle \otimes|0\rangle=0 .
$$

Since we may regard $\omega$ as an element of $H^{0}\left(C, \omega_{C}\left(* \sum_{j=1}^{N} Q_{j}\right)\right)$ having a zero at $P$, we get by using (3.18) that

$$
\begin{gathered}
\left\langle\widetilde{\Phi} \mid \rho_{+}\left(\psi\left[\omega_{+}\right]\right) 0 \otimes 0 \otimes u\right\rangle+\left\langle\widetilde{\Phi} \mid \rho_{-}\left(\psi\left[\omega_{-}\right]\right) 0 \otimes 0 \otimes u\right\rangle+\sum_{j=1}^{N}\left\langle\widetilde{\Phi} \mid \rho_{j}\left(\psi\left[\omega_{j}\right]\right) 0 \otimes 0 \otimes u\right\rangle \\
=-\sum_{k=1}^{N}\left\{\sum_{j=1}^{N}\left\langle\Phi \mid \rho_{j}\left(\psi\left[\omega_{j}\right]\right) \rho_{k}\left[f_{k}\right] u\right\rangle\right\}=0,
\end{gathered}
$$

by the first gauge condition for $\langle\Phi|$.

Next let

$$
\begin{aligned}
& h_{+}=a_{0}+a_{1} z+a_{2} z^{2}+\cdots \\
& h_{-}=b_{0}+b_{1} w+b_{2} w^{2}+\cdots
\end{aligned}
$$


be the Taylor expansion of $h \in H^{0}\left(\widetilde{C}, \mathcal{O}_{\widetilde{C}}\left(* \sum_{j=1}^{N} Q_{j}\right)\right)$ at $P_{ \pm}$. Then we have that

$$
\rho_{+}\left(\bar{\psi}\left[h_{+}\right]\right)|0\rangle \otimes|0\rangle=0, \quad \rho_{-}\left(\bar{\psi}\left[h_{-}\right]\right)|0\rangle \otimes|0\rangle=0 .
$$

If $a_{0}=b_{0}$ then $h \in H^{0}\left(C, \mathcal{O}_{C}\left(* \sum_{j=1}^{N} Q_{j}\right)\right)$. Then by similar arguments as above, we can show that

$$
\left\langle\widetilde{\Phi} \mid \rho_{+}\left(\bar{\psi}\left[h_{+}\right]\right) 0 \otimes 0 \otimes u\right\rangle+\left\langle\widetilde{\Phi} \mid \rho_{-}\left(\bar{\psi}\left[h_{-}\right]\right) 0 \otimes 0 \otimes u\right\rangle+\sum_{j=1}^{N}\left\langle\widetilde{\Phi} \mid \rho_{j}\left(\bar{\psi}\left[h_{j}\right]\right) 0 \otimes 0 \otimes u\right\rangle=0 .
$$

If $a_{0} \neq b_{0}$, then by subtracting from $f$ the constant $b_{0}$ and multiplying by a constant, we may assume that $h$ has Taylor expansions of type (3.16) and (3.17). Then by the above argument we have that

$$
\langle\widetilde{\Phi} \mid 0 \otimes 0 \otimes u\rangle=\sum_{k=1}^{N}\left\langle\Phi \mid \rho_{k}\left(\bar{\psi}\left[h_{k}\right]\right) u\right\rangle
$$

Then we have that

$$
\begin{aligned}
& \sum_{j=1}^{N}\left\langle\widetilde{\Phi} \mid 0 \otimes 0 \otimes \rho_{j}\left(\bar{\psi}\left[h_{j}\right]\right) u\right\rangle=\sum_{j=1}^{N} \sum_{k=1}^{N}\left\langle\Phi \mid \rho_{j}\left(\bar{\psi}\left[h_{j}\right]\right) \rho_{k}\left(\bar{\psi}\left[h_{k}\right]\right) u\right\rangle \\
& \quad=-\sum_{k=1}^{N} \sum_{j=1}^{N}\left\langle\Phi \mid \rho_{j}\left(\bar{\psi}\left[h_{j}\right]\right) \rho_{k}\left(\bar{\psi}\left[h_{k}\right]\right) u\right\rangle=-\sum_{j=1}^{N}\left\langle\widetilde{\Phi} \mid 0 \otimes 0 \otimes \rho_{j}\left(\bar{\psi}\left[h_{j}\right]\right) u\right\rangle,
\end{aligned}
$$

since we have that

$$
\rho_{j}\left(\bar{\psi}\left[h_{j}\right]\right) \rho_{k}\left(\bar{\psi}\left[h_{k}\right]\right)|u\rangle=-\rho_{k}\left(\bar{\psi}\left[h_{k}\right]\right) \rho_{j}\left(\bar{\psi}\left[h_{j}\right]\right)|u\rangle .
$$

Hence

$$
\sum_{j=1}^{N}\left\langle\widetilde{\Phi} \mid 0 \otimes 0 \otimes \rho_{j}\left(\bar{\psi}\left[h_{j}\right]\right) u\right\rangle=0 .
$$

Thus we conclude that

$$
\left\langle\widetilde{\Phi} \mid \rho_{+}\left(\bar{\psi}\left[h_{+}\right]\right) 0 \otimes 0 \otimes u\right\rangle+\left\langle\widetilde{\Phi} \mid \rho_{-}\left(\bar{\psi}\left[h_{-}\right]\right) 0 \otimes 0 \otimes u\right\rangle+\sum_{j=1}^{N}\left\langle\widetilde{\Phi} \mid \rho_{j}\left(\bar{\psi}\left[h_{j}\right]\right) 0 \otimes 0 \otimes u\right\rangle=0 .
$$

Now we can apply Theorem 3.4 twice and obtained the desired result.

Remark 3.1. The choice of $\left|0_{+,-}\right\rangle$is not unique. Any non-zero multiple of $\left|0_{+,-}\right\rangle$gives a natural isomorphism of the above theorem. But the above normalization of $\left|0_{+,-}\right\rangle$will be seen to be compatible with the preferred elements of the ghost vacua on a nodal curve and on its normalization. For details see Theorem 6.5 below. 


\section{Sheaf of Ghost Vacua}

The arguments of this section are almost identical to those in [U2], section 4, but for the readers' convenience we shall repeat them here in this abelian case.

Let

$$
\mathfrak{F}=\left(\pi: \mathcal{C} \rightarrow \mathcal{B} ; s_{1}, \ldots, s_{N} ; \xi_{1}, \ldots, \xi_{N}\right)
$$

be a family of $N$-pointed semi-stable curves with formal coordinates. That is $\mathcal{C}$ and $\mathcal{B}$ are complex manifolds, $\pi$ is a proper holomorphic mapping, and for each point $b \in \mathcal{B}$, $\mathfrak{F}(b)=\left(C_{b}=\pi^{-1}(b) ; s_{1}(b), \ldots, s_{N}(b) ; \xi_{1}, \ldots, \xi_{N}\right)$ is an $N$-pointed semi-stable curve with formal coordinates. We let $\Sigma$ be the locus of double points of the fibers of $\mathfrak{F}$ and let $D$ be $\pi(\Sigma)$. Note that $\Sigma$ is a non-singular submanifold of codimension two in $\mathcal{C}$, and $D$ is a divisor in $\mathcal{B}$ whose irreducible components $D_{i}, i=1,2, \ldots, m^{\prime}$ are non-singular.

In this section we use the following notation freely.

$$
S_{j}=s_{j}(\mathcal{B}), \quad S=\sum_{j=1}^{N} S_{j} .
$$

Put

$$
\mathcal{F}_{N}(\mathcal{B})=\mathcal{F}_{N} \otimes_{\mathbf{C}} \mathcal{O}_{\mathcal{B}}, \quad \mathcal{F}_{N}^{\dagger}(\mathcal{B})=\mathcal{O}_{\mathcal{B}} \otimes_{\mathbf{C}} \mathcal{F}_{N}^{\dagger}
$$

Definition 4.1. We define the subsheaf $\mathcal{V}^{\dagger}{ }_{\mathrm{ab}}(\mathfrak{F})$ of $\mathcal{F}_{N}^{\dagger}(\mathcal{B})$ by the gauge conditions:

$$
\begin{aligned}
& \sum_{j=1}^{N}\langle\Phi| \psi\left[\omega_{j}\right]=0, \quad \text { for all } \omega \in \pi_{*}\left(\omega_{\mathcal{C} / \mathcal{B}}(* S)\right), \\
& \sum_{j=1}^{N}\langle\Phi| \bar{\psi}\left[f_{j}\right]=0, \quad \text { for all } f \in \pi_{*} \mathcal{O}_{\mathcal{C}}(* S) .
\end{aligned}
$$

where $\omega_{j}$ and $f_{j}$ are the Laurent expansion of $\omega$ and $f$ along $S_{j}$ with respect to the coordinate $\xi_{j}$.

The sheaf $\mathcal{V}^{\dagger}{ }_{\mathrm{ab}}(\mathfrak{F})$ is called the sheaf of $(j=0)$ ghost vacua or the sheaf of abelian vacua of the family $\mathfrak{F}$. Similarly the sheaf $\mathcal{V}_{\mathrm{ab}}(\mathfrak{F})$ of $(j=0)$ dual ghost vacua of the family is defined by

$$
\mathcal{V}_{\mathrm{ab}}(\mathfrak{F})=\mathcal{F}_{N}(\mathcal{B}) / \mathcal{F}_{\mathrm{ab}}(\mathfrak{F}) .
$$

where $\mathcal{F}_{\text {ab }}(\mathfrak{F})$ is the $\mathcal{O}_{\mathcal{B}}$-submodule of $\mathcal{F}_{N}(\mathcal{B})$ given by $\mathcal{F}_{\text {ab }}(\mathfrak{F})=\mathcal{F}_{\text {ab }}^{0}(\mathfrak{F})+\mathcal{F}_{\text {ab }}^{1}(\mathfrak{F})$, where $\mathcal{F}_{\text {ab }}^{0}(\mathfrak{F})$ is the span of $\bar{\psi}[f] \mathcal{F}_{N}(\mathcal{B})$ for all $f \in \pi_{*} \mathcal{O}_{\mathcal{C}}(* S)$ and $\mathcal{F}_{\text {ab }}^{1}(\mathfrak{F})$ is the span of $\psi[\omega] \mathcal{F}_{N}(\mathcal{B})$ for all $\omega \in \pi_{*} \omega_{\mathcal{C} / \mathcal{B}}(* S)$.

Note that we have

$$
\mathcal{V}^{\dagger}{ }_{\mathrm{ab}}(\mathfrak{F})=\underline{\operatorname{Hom}}_{\mathcal{O}_{\mathcal{B}}}\left(\mathcal{V}_{\mathrm{ab}}(\mathfrak{F}), \mathcal{O}_{\mathcal{B}}\right)
$$

Moreover, by the right exactness of the tensor product we have that

$$
\mathcal{V}_{\mathrm{ab}}(\mathfrak{F}) \otimes_{\mathcal{O}_{\mathcal{B}}} \mathcal{O}_{\mathcal{B}, b} / \mathfrak{m}_{b} \cong \mathcal{V}_{\mathrm{ab}}(\mathfrak{F}(b))
$$

Proposition 4.1. The sheaves $\mathcal{V}_{\mathrm{ab}}(\mathfrak{F})$ and $\mathcal{V}^{\dagger} \mathrm{ab}(\mathfrak{F})$ are coherent $\mathcal{O}_{\mathcal{B}}$-modules. 
Proof First we need to introduce a filtration on $\mathcal{F}$. Note that any element of $\mathcal{F}$ is a finite linear combination of elements of the form

$$
|u\rangle=\bar{\psi}_{\mu_{1}} \bar{\psi}_{\mu_{2}} \cdots \bar{\psi}_{\mu_{r}} \psi_{\nu_{s}} \psi_{\nu_{s-1}} \cdots \psi_{\nu_{1}}|0\rangle
$$

with $\mu_{1}<\mu_{2}<\cdots<\mu_{r}<0, \nu_{1}<\nu_{2}<\cdots<\nu_{s}<0$. Let us define an alternative degree $d(|u\rangle)$ by

$$
d(|u\rangle)=\sum_{i=1}^{r}\left(-\mu_{i}+\frac{1}{2}\right)+\sum_{j=1}^{s}\left(-\nu_{j}-\frac{1}{2}\right) .
$$

Note that this alternative degree $d(|u\rangle)$ is different form the degree of the Maya diagram associated to $|u\rangle$. We shall only need this alternative degree in this proof and here we shall just refer to it as the degree. Let $\mathcal{F}_{d}$ be the subspace of $\mathcal{F}$ spanned by the elements of degree $d$. The filtration $F_{\bullet}$ on $\mathcal{F}$ is defined by

$$
F_{p} \mathcal{F}=\bigoplus_{d \leq p} \mathcal{F}_{d}
$$

Note that $F_{p} \mathcal{F}=0$ for $p<0$ and $F_{0} \mathcal{F}=\mathbf{C}|0\rangle+\mathbf{C}|-1\rangle$. By definition we have that

$$
\psi_{\nu} F_{p} \mathcal{F} \subset F_{p-\nu-1 / 2} \mathcal{F}, \quad \bar{\psi}_{\mu} F_{p} \mathcal{F} \subset F_{p-\mu+1 / 2} \mathcal{F} .
$$

Moreover we have

$$
G r_{\bullet}^{F} \mathcal{F}=\bigoplus \mathcal{F}_{d}
$$

Also let us introduce filtrations on $\mathbf{C}((\xi))$ and $\mathbf{C}(\xi)) d \xi$ by

$$
\left.F_{p} \mathbf{C}((\xi))=\mathbf{C}[[\xi]] \xi^{-p}, \quad F_{p} \mathbf{C}(\xi)\right) d \xi=\mathbf{C}[[\xi]] \xi^{-p} d \xi .
$$

Then we have that

$$
G r_{\bullet}^{F} \mathbf{C}((\xi))=\mathbf{C}\left[\xi, \xi^{-1}\right], \quad G r_{\bullet}^{F} \mathbf{C}((\xi)) d \xi=\mathbf{C}\left[\xi, \xi^{-1}\right] d \xi .
$$

Put

$$
\begin{aligned}
\psi\left[F_{p} \mathbf{C}((\xi)) d \xi\right] & =\left\{\bar{\psi}[\omega(\xi)] \mid \omega(\xi) \in F_{p} \mathbf{C}((\xi)) d \xi\right\} \\
\bar{\psi}\left[F_{p} \mathbf{C}((\xi))\right] & =\left\{\bar{\psi}[f(\xi)] \mid f(\xi) \in F_{p} \mathbf{C}((\xi))\right\}
\end{aligned}
$$

By (4.3) it is easy to show that following facts

$$
\psi\left[F_{p} \mathbf{C}((\xi)) d \xi\right] F_{q} \mathcal{F} \subset F_{p+q} \mathcal{F}, \quad \bar{\psi}\left[F_{p} \mathbf{C}((\xi))\right] F_{q} \mathcal{F} \subset F_{p+q} \mathcal{F} .
$$

let $\mathfrak{X}=(C, Q, \xi)$ be a one-pointed curve of genus $g$ with coordinate. The filtrations $F_{\bullet}$ on $\mathbf{C}((\xi))$ and $\mathbf{C}((\xi)) d \xi$ induce the ones on $H^{0}\left(C, \mathcal{O}_{C}(* Q)\right)$ and $H^{0}\left(C, \omega_{C}(* Q)\right)$ and we have that

$$
F_{p} H^{0}\left(C, \mathcal{O}_{C}(* Q)\right)=H^{0}\left(C, \mathcal{O}_{C}(p Q)\right), \quad F_{p} H^{0}\left(C, \omega_{C}(* Q)\right)=H^{0}\left(C, \omega_{C}(p Q)\right) .
$$

Thus the filtrations are compatible with the actions of $\psi[\omega]$ and $\bar{\psi}[f]$ to $\mathcal{F}$ for $\omega \in$ $H^{0}\left(C, \omega_{C}(* Q)\right)$ and $f \in H^{0}\left(C, \mathcal{O}_{C}(* Q)\right)$. Hence the inclusion $\mathcal{F}(\mathfrak{X}) \subset \mathcal{F}$ is compatible with the filtrations and the quotient space $\mathcal{V}(\mathfrak{X})$ has the induced filtration $F_{\bullet}$. Thus we conclude that

$$
G r_{\bullet}^{F} \mathcal{V}(\mathfrak{X})=G r_{\bullet}^{F} \mathcal{F} / G r_{\bullet}^{F} \mathcal{F}(\mathfrak{X})
$$


Let $1=n_{1}<n_{2}<\cdots<n_{g}=2 g-1$ be the Weierstrass gap values of the curve $C$ at $Q$. Then $G r_{\bullet}^{F} H^{0}\left(C, \mathcal{O}_{C}(* Q)\right) \subset \mathbf{C}\left[\xi^{-1}\right]$ does not contain $\xi^{-n_{i}}, i=1, \ldots, g$ but contain $\xi^{-n}, n \neq n_{i}, i=1, \ldots, g$. Also $G r_{\bullet}^{F} H^{0}\left(C, \omega_{C}(* Q)\right)$ contains all $\xi^{-n} d \xi, n \leq 0$. Thus

$$
|u\rangle=\bar{\psi}_{\mu_{1}} \bar{\psi}_{\mu_{2}} \cdots \bar{\psi}_{\mu_{r}} \psi_{\nu_{s}} \psi_{\nu_{s-1}} \cdots \psi_{\nu_{1}}|0\rangle
$$

may not be in $G r_{\bullet}^{F} \mathcal{V}(\mathfrak{X})$ if $s=0$ and $\left\{\mu_{1}, \ldots, \mu_{r}\right\} \subset\left\{-n_{2}+1 / 2, \ldots,-n_{g}+1 / 2\right\}$. Thus $G r^{F} \mathcal{V}(\mathfrak{X})$ is of finite dimensional. Moreover if $V$ is the subspace spanned by

$$
\bar{\psi}_{-m_{1}+1 / 2} \bar{\psi}_{-m_{2}+1 / 2} \cdots \bar{\psi}_{-m_{r}+1 / 2}|0\rangle
$$

with $2 g-1 \geq m_{1}>m_{2}>\cdots>m_{r} \geq 0$ where $1 \leq r \leq 2 g$, then $V$ is a finite dimensional vector space and the natural linear map $V \rightarrow G r_{\bullet} \mathcal{V}(\mathfrak{X})$ is surjective.

Now for a family

$$
\mathfrak{F}=(\pi: \mathcal{C} \rightarrow \mathcal{B} ; s ; \xi)
$$

of one-pointed curves of genus $g$ with coordinate, we can introduce filtrations on $\mathcal{F}(\mathcal{B})$, $\mathcal{F}(\mathfrak{F}), \pi_{*} \mathcal{O}_{\mathcal{C}}(* S)$ and $\pi_{*} \omega_{\mathcal{C} / \mathcal{B}}(* S)$ of $\mathcal{O}_{\mathcal{B}}$-submodules in the same way as above so that we can introduce a filtration on $\mathcal{V}(\mathfrak{F})$. Moreover, there is an $\mathcal{O}_{\mathcal{B}}$-module homomorphism $V \otimes_{\mathbf{C}} \mathcal{O}_{\mathcal{B}} \rightarrow G r_{\bullet}^{F} \mathcal{V}(\mathfrak{F})$ which is surjective by virtue of (4.2) and the above argument. Thus $G r_{\bullet}^{F} \mathcal{V}(\mathfrak{F})$ is a coherent $\mathcal{O}_{\mathcal{B}}$-module. Therefore, $\mathcal{V}(\mathfrak{F})$ is also a coherent $\mathcal{O}_{\mathcal{B}}$-module. This proves the proposition for the one-pointed case. The general case can be proved similarly or we can use Theorem 3.4 to reduce the general case to the one-pointed case.

QED

Let us now show the local freeness of the sheaves $\mathcal{V}_{\mathrm{ab}}(\mathfrak{F})$ and $\mathcal{V}^{\dagger}{ }_{\mathrm{ab}}(\mathfrak{F})$. For that purpose we first introduce a certain $\mathcal{O}_{\mathcal{B}}$-submodule $\mathcal{L}(\mathfrak{F})$ of

$$
\bigoplus_{j=1}^{N} \mathcal{O}_{\mathcal{B}}\left(\left(\xi_{j}^{-1}\right)\right) \frac{d}{d \xi_{j}}
$$

and an action of $\mathcal{L}(\mathfrak{F})$ on the sheaves $\mathcal{V}_{\mathrm{ab}}(\mathfrak{F})$ and $\mathcal{V}^{\dagger}{ }_{\mathrm{ab}}(\mathfrak{F})$ as first order twisted differential operators. This action will also be used to define a connection on the sheaves $\mathcal{V}_{\text {ab }}(\mathfrak{F})$ and $\mathcal{V}^{\dagger}{ }_{a b}(\mathfrak{F})$.

First recall that we have an exact sequence

$$
0 \rightarrow \Theta_{\mathcal{C} / \mathcal{B}}(-S) \rightarrow \Theta_{\mathcal{C} / \mathcal{B}}(m S) \rightarrow \bigoplus_{j=1}^{N} \bigoplus_{k=0}^{m} \mathcal{O}_{\mathcal{B}} \xi_{j}^{-k} \frac{d}{d \xi_{j}} \rightarrow 0
$$

for any positive integer $m$. From this exact sequence we obtain the exact sequence

$$
0 \rightarrow \pi_{*}\left(\Theta_{\mathcal{C} / \mathcal{B}}(m S)\right) \stackrel{b_{m}}{\longrightarrow} \bigoplus_{j=1}^{N} \bigoplus_{k=0}^{m} \mathcal{O}_{\mathcal{B}} \xi_{j}^{-k} \frac{d}{d \xi_{j}} \stackrel{\vartheta_{m}}{\longrightarrow} R^{1} \pi_{*} \Theta_{\mathcal{C} / \mathcal{B}}(-S) \rightarrow 0 .
$$

Hence, we have the following exact sequence of $\mathcal{O}_{\mathcal{B}}$-modules

$$
0 \rightarrow \pi_{*}\left(\Theta_{\mathcal{C} / \mathcal{B}}(* S)\right) \stackrel{b}{\longrightarrow} \bigoplus_{j=1}^{N} \mathcal{O}_{\mathcal{B}}\left[\xi_{j}^{-1}\right] \frac{d}{d \xi_{j}} \stackrel{\vartheta}{\longrightarrow} R^{1} \pi_{*} \Theta_{\mathcal{C} / \mathcal{B}}(-S) \rightarrow 0
$$


Note that the mappings $b$ and $b_{m}$ correspond to the Laurent expansions with respect to $\xi_{j}$ up to zero'th order. To define the first order differential operators acting on the sheaves $\mathcal{V}_{\mathrm{ab}}(\mathfrak{F})$ and $\mathcal{V}^{\dagger}{ }_{\mathrm{ab}}(\mathfrak{F})$, we need to rewrite the exact sequence (4.5) in the following way.

There is an exact sequence

$$
0 \rightarrow \Theta_{\mathcal{C} / \mathcal{B}} \rightarrow \Theta_{\mathcal{C}} \stackrel{d \pi}{\longrightarrow} \pi^{*} \Theta_{\mathcal{B}} \rightarrow 0
$$

where $\Theta_{\mathcal{C} / \mathcal{B}}$ is the sheaf of vector fields tangent to the fibers of $\pi$. Put

$$
\Theta_{\mathcal{C}, \pi}^{\prime}=d \pi^{-1}\left(\pi^{-1} \Theta_{\mathcal{B}}(-\log D)\right) .
$$

Hence, $\Theta_{\mathcal{C}, \pi}^{\prime}$ is the sheaf of vector field on $\mathcal{C}$ tangent to $\Sigma$ whose horizontal components are constant along the fibers of $\pi$. That is, $\Theta_{\mathcal{C}, \pi}^{\prime}$ consists of germs of holomorphic vector fields of the form

$$
a(z, u) \frac{\partial}{\partial z}+\sum_{i=1}^{m^{\prime}} b_{i}(u) u_{i} \frac{\partial}{\partial u_{i}}+\sum_{i=m^{\prime}+1}^{n} b_{i}(u) \frac{\partial}{\partial u_{i}},
$$

where $\left(z, u_{1}, \ldots, u_{n}\right)$ is a system of local coordinates such that the mapping $\pi$ is expressed as the projection

$$
\pi\left(z, u_{1}, \ldots, u_{n}\right)=\left(u_{1}, \ldots, u_{n}\right)
$$

and $\pi(\Sigma)=D$ is given by the equation

$$
u_{1} \cdot u_{2} \cdots u_{m^{\prime}}=0
$$

More generally, we can define the sheaf $\Theta_{\mathcal{C}}^{\prime}(m S)_{\pi}$ as the one consisting of germs of meromorphic vector fields of the form

$$
A(z, u) \frac{\partial}{\partial z}+\sum_{i=1}^{m^{\prime}} B_{i}(u) u_{i} \frac{\partial}{\partial u_{i}}+\sum_{i=m^{\prime}+1}^{n} B_{i}(u) \frac{\partial}{\partial u_{i}},
$$

where $A(z, u)$ has a poles of order at most $m$ along $S$. Now we have an exact sequence

$$
0 \rightarrow \Theta_{\mathcal{C} / \mathcal{B}}(m S) \rightarrow \Theta_{\mathcal{C}}^{\prime}(m S)_{\pi} \stackrel{d \pi}{\longrightarrow} \pi^{-1} \Theta_{\mathcal{B}}(-\log D) \rightarrow 0 .
$$

Note that $\Theta_{\mathcal{C}}^{\prime}(m S)_{\pi}$ has the structure of a sheaf of Lie algebras by the usual bracket operation of vector fields and the above exact sequence is one of sheaves of Lie algebras.

For $m>\frac{1}{N}(2 g-2)$, by (4.6) we have an exact sequence of $\mathcal{O}_{\mathcal{B}}$-modules.

$$
0 \rightarrow \pi_{*} \Theta_{\mathcal{C} / \mathcal{B}}(m S) \longrightarrow \pi_{*} \Theta_{\mathcal{C}}^{\prime}(m S)_{\pi} \stackrel{d \pi}{\longrightarrow} \Theta_{\mathcal{B}}(-\log D) \rightarrow 0,
$$

which is also an exact sequence of sheaves of Lie algebras. Taking $m \rightarrow \infty$ we obtain the exact sequence

$$
0 \rightarrow \pi_{*} \Theta_{\mathcal{C} / \mathcal{B}}(* S) \longrightarrow \pi_{*} \Theta_{\mathcal{C}}^{\prime}(* S)_{\pi} \stackrel{d \pi}{\longrightarrow} \Theta_{\mathcal{B}}(-\log D) \rightarrow 0 .
$$


The exact sequences (4.5) and (4.8) are related by the following commutative diagram.

$$
\begin{aligned}
& 0 \rightarrow \pi_{*} \Theta_{\mathcal{C} / \mathcal{B}}(* S) \quad \rightarrow \quad \pi_{*} \Theta_{\mathcal{C}}^{\prime}(* S)_{\pi} \quad \stackrel{d \pi}{\longrightarrow} \quad \Theta_{\mathcal{B}}(-\log D) \quad \rightarrow \quad 0 \\
& \| \quad \downarrow p \quad \downarrow \rho \\
& 0 \rightarrow \pi_{*} \Theta_{\mathcal{C} / \mathcal{B}}(* S) \rightarrow \bigoplus_{j=1}^{N} \mathcal{O}_{\mathcal{B}}\left[\xi_{j}^{-1}\right] \frac{d}{d \xi_{j}} \stackrel{\vartheta}{\longrightarrow} R^{1} \pi_{*} \Theta_{\mathcal{C} / \mathcal{B}}(-S) \rightarrow 0,
\end{aligned}
$$

where $\rho$ is the Kodaira-Spencer mapping of the family $\mathfrak{F}$ and $p$ is given by taking the non-positive degree part of the $\frac{d}{d \xi_{j}}$ part of the Laurent expansions of the vector fields in $\pi_{*} \Theta_{\mathcal{C}}^{\prime}(* S)_{\pi}$ at $s_{j}(\mathcal{B})$.

In the following, for simplicity we assume that the Kodaira-Spencer mapping $\rho$ is an injective homomorphism of $\mathcal{O}_{\mathcal{B}}$-modules. Therefore, $p$ is an injection. Let

$$
\widetilde{p}: \pi_{*} \Theta_{\mathcal{C}}^{\prime}(* S)_{\pi} \longrightarrow \bigoplus_{j=1}^{N} \mathcal{O}_{\mathcal{B}}\left(\left(\xi_{j}\right)\right) \frac{d}{d \xi_{j}}
$$

be defined by taking the $\frac{d}{d \xi_{j}}$ part of the Laurent expansions at $s_{j}(\mathcal{B})$. Since $p$ is injective, $\widetilde{p}$ is injective. Put

$$
\mathcal{L}(\mathfrak{F}):=\widetilde{p}\left(\pi_{*} \Theta_{\mathcal{C}}^{\prime}(* S)_{\pi}\right) .
$$

Then, we have the exact sequence

$$
0 \rightarrow \pi_{*} \Theta_{\mathcal{C} / \mathcal{B}}(* S) \longrightarrow \mathcal{L}(\mathfrak{F}) \stackrel{\theta}{\longrightarrow} \Theta_{\mathcal{B}}(-\log D) \rightarrow 0
$$

of $\mathcal{O}_{\mathcal{B}}$-modules. This is the exact sequence corresponding to (4.5).

The Lie bracket $[,]_{d}$ on $\mathcal{L}(\mathfrak{F})$ is obtained from the bracket on $\pi_{*} \Theta_{\mathcal{C}}^{\prime}(* S)_{\pi}$ by the mapping $\widetilde{p}$. Thus, for $\vec{\ell}, \vec{m} \in \mathcal{L}(\mathfrak{F})$ we have that

$$
[\vec{\ell}, \vec{m}]_{d}=[\vec{\ell}, \vec{m}]_{0}+\theta(\vec{\ell})(\vec{m})-\theta(\vec{m})(\vec{\ell})
$$

where $[\cdot, \cdot]_{0}$ is the usual bracket of formal vector fields and the action of $\theta(\vec{\ell})$ on

$$
\vec{m}=\left(m_{1} \frac{d}{d \xi_{1}}, \ldots, m_{N} \frac{d}{d \xi_{N}}\right)
$$

is defined by

$$
\left(\theta(\vec{\ell})\left(m_{1}\right) \frac{d}{d \xi_{1}}, \ldots, \theta(\vec{\ell})\left(m_{N}\right) \frac{d}{d \xi_{N}}\right) .
$$

Then, the exact sequence (4.10) is also an exact sequence of sheaves of Lie algebras.

Let us define an action of $\mathcal{L}(\mathfrak{F})$ on $\mathcal{F}_{N}(\mathcal{B})$.

Definition 4.2. For $\vec{\ell}=\left(\ell_{1}, \ldots, \ell_{N}\right) \in \mathcal{L}(\mathfrak{F})$, the action of $D(\vec{\ell})$ on $\mathcal{F}_{N}(\mathcal{B})$ is defined by

$$
D(\vec{\ell})(F \otimes|u\rangle)=\theta(\vec{\ell})(F) \otimes|u\rangle-F \cdot\left(\sum_{j=1}^{N} \rho_{j}\left(T\left[\ell_{j}\right]\right)\right)|u\rangle,
$$


where

$$
F \in \mathcal{O}_{\mathcal{B}}, \quad|u\rangle \in \mathcal{F}_{N},
$$

and

$$
T[\ell]=\operatorname{Res}_{z=0}(T(z) \ell(z) d z) .
$$

The following proposition can be proved in the same manner as Proposition 4.2.2 of [U2].

Proposition 4.2. The action $D(\vec{\ell})$ of $\vec{\ell} \in \mathcal{L}(\mathfrak{F})$ on $\mathcal{F}_{N}(\mathcal{B})$ defined above has the following properties.

1. For any $F \in \mathcal{O}_{\mathcal{B}}$ we have

$$
D(F \vec{\ell})=F D(\vec{\ell})
$$

2. For $\vec{\ell}, \vec{m} \in \mathcal{L}(\mathfrak{F})$ we have

$$
[D(\vec{\ell}), D(\vec{m})]=D\left([\vec{\ell}, \vec{m}]_{d}\right)-\frac{1}{6} \sum_{j=1}^{N} \operatorname{Res}_{\xi_{j}=0}\left(\frac{d^{3} \ell_{j}}{d \xi_{j}^{3}} m_{j} d \xi_{j}\right) \cdot i d .
$$

3. For $F \in \mathcal{O}_{\mathcal{B}}$ and $|\phi\rangle \in \mathcal{F}_{N}(\mathcal{B})$ we have

$$
D(\vec{\ell})(F|\phi\rangle)=(\theta(\vec{\ell})(F))|\phi\rangle+F D(\vec{\ell})|\phi\rangle .
$$

Namely, $D(\vec{\ell})$ is a first order differential operator, if $\theta(\vec{\ell}) \neq 0$.

We define the dual action of $\mathcal{L}(\mathfrak{F})$ on $\mathcal{F}_{N}^{\dagger}(\mathcal{B})$ by

$$
D(\vec{\ell})(F \otimes\langle\Phi|)=\theta(\vec{\ell})(F) \otimes\langle\Phi|+\sum_{j=1}^{N} F \cdot\langle\Phi| \rho_{j}\left(T\left[\ell_{j}\right]\right) .
$$

where

$$
F \in \mathcal{O}_{\mathcal{B}}, \quad\langle\Phi| \in \mathcal{F}_{N}^{\dagger}(\mathcal{B}) .
$$

Then, for any $|u\rangle \in \mathcal{F}_{N}(\mathcal{B})$ and $\langle\Phi| \in \mathcal{F}_{N}^{\dagger}(\mathcal{B})$, we have

$$
\{D(\vec{\ell})\langle\Phi|\}|\widetilde{\Phi}\rangle+\langle\Phi|\{D(\vec{\ell})|\widetilde{\Phi}\rangle\}=\theta(\vec{\ell})\langle\Phi \mid \widetilde{\Phi}\rangle .
$$

This agrees with the usual definition of the dual connection.

Now we shall show that the operator $D(\vec{\ell})$ acts on $\mathcal{V}_{\mathrm{ab}}(\mathfrak{F})$.

Proposition 4.3. For any $\vec{\ell} \in \mathcal{L}(\mathfrak{F})$ we have

$$
D(\vec{\ell})\left(\mathcal{F}_{\mathrm{ab}}(\mathfrak{F})\right) \subset \mathcal{F}_{\mathrm{ab}}(\mathfrak{F}) .
$$

Hence, $D(\vec{\ell})$ operates on $\mathcal{V}_{\mathrm{ab}}(\mathfrak{F})$. Moreover, it is a first order differential operator, if $\theta(\vec{\ell}) \neq 0$. 
Proof. An element of $\mathcal{F}_{\mathrm{ab}}(\mathfrak{F})$ is an $\mathcal{O}_{\mathcal{B}}$-linear combination of elements of the form

$$
F \otimes\left(\sum_{j=1}^{N} \rho_{j}\left(\psi\left[\omega_{j}\right]\right)|u\rangle\right), \quad F^{\prime} \otimes\left(\sum_{j=1}^{N} \rho_{j}\left(\bar{\psi}\left[f_{j}\right]\right)|u\rangle\right)
$$

where

$$
F, F^{\prime} \in \mathcal{O}_{\mathcal{B}}, \quad \omega \in \pi_{*}\left(\omega_{\mathcal{C} / \mathcal{B}}(* S)\right), \quad f \in \pi_{*} \mathcal{O}_{\mathcal{C}}(* S), \quad|u\rangle \in \mathcal{F}_{N}
$$

and $f_{j}$ is the Laurent expansion of $f$ along $S_{j}=s_{j}(\mathcal{B})$ with respect to the coordinate $\xi_{j}$ and likewise for $\omega$. First we shall show the following equality as operators on $\mathcal{F}_{N}(\mathcal{B})$

$$
\left[D(\vec{\ell}), \sum_{j=1}^{N} \rho_{j}\left(\bar{\psi}\left[f_{j}\right]\right)\right]=\sum_{j=1}^{N} \rho_{j}\left(\bar{\psi}\left[\theta(\vec{\ell})\left(f_{j}\right)+\underline{\ell}_{j}\left(f_{j}\right)\right]\right)
$$

where $\theta(\vec{\ell})$ operates on the coefficients of $f_{j}$. By Proposition 4.2, (3) it is enough to show the equality (4.15) as operators on $\mathcal{F}_{N}$. For $|u\rangle \in \mathcal{F}_{N}$, by (4.12) we have

$$
\begin{aligned}
D(\vec{\ell}) & \left(\sum_{j=1}^{N} \rho_{j}\left(\bar{\psi}\left[f_{j}\right]\right)|u\rangle\right)-\sum_{j=1}^{N} \rho_{j}\left(\bar{\psi}\left[f_{j}\right]\right)(D(\vec{\ell})|u\rangle) \\
& =\sum_{j=1}^{N}\left\{\rho_{j}\left(\bar{\psi}\left[\theta(\vec{\ell})\left(f_{j}\right)\right]\right)-\rho_{j}\left(T\left[\underline{\ell}_{j}\right]\right) \rho_{j}\left(\bar{\psi}\left[f_{j}\right]\right)\right\}|u\rangle+\sum_{j=1}^{N} \rho_{j}\left(\bar{\psi}\left[f_{j}\right]\right) \rho_{j}\left(T\left[\underline{\ell}_{j}\right]\right)|u\rangle \\
& \left.=\sum_{j=1}^{N}\left\{\rho_{j}\left(\bar{\psi}\left[\theta(\vec{\ell})\left(f_{j}\right)+\underline{\ell}_{j}\left(f_{j}\right)\right)\right\}\right]\right)|u\rangle .
\end{aligned}
$$

This implies equation (4.15). Now $\theta(\vec{\ell})\left(f_{j}\right)+\underline{\ell}_{j}\left(f_{j}\right)$ is nothing but the Laurent expansion at $s_{j}(\mathcal{B})$ of the meromorphic function $\tau(h)$ where $\tau=\widetilde{p}^{-1}(\vec{\ell}) \in \pi_{*}\left(\Theta_{\mathcal{C}}^{\prime}(* S)_{\pi}\right)$. Hence, we have the result that $D(\vec{\ell}) \mathcal{F}_{(0,1}^{0}(\mathfrak{F}) \subset \mathcal{F}_{(0,1}^{0}(\mathfrak{F})$.

Next let us consider $\omega \in \pi_{*}\left(\omega_{\mathcal{C} / \mathcal{B}}(* S)\right)$. For $\tau=\widetilde{p}^{-1}(\vec{\ell}) \in \pi_{*}\left(\Theta_{\mathcal{C}}^{\prime}(* S)_{\pi}\right)$ put

$$
\widetilde{\omega}=\frac{d}{d t}\left(\exp \{t \tau\}^{*}(\omega)\right)
$$

Then the Laurent expansion of $\widetilde{\omega}$ along $S_{j}$ is written as

$$
\widetilde{\omega}_{j}=\ell_{j}\left(A_{j}\left(\xi_{j}\right)\right)+\omega^{\left(\ell_{j}\right)},
$$

where $\omega_{j}=A_{j}\left(\xi_{j}\right) d \xi_{j}$ and

$$
\omega^{\left(\ell_{j}\right)}=A_{j}\left(\xi_{j}\right) \frac{d \ell_{j}\left(\xi_{j}\right)}{d \xi_{j}} .
$$

Now we are ready to prove the following equality as operators on $\mathcal{F}_{N}(\mathcal{B})$

$$
\left[D(\vec{\ell}), \sum_{j=1}^{N} \rho_{j}\left(\psi\left[\omega_{j}\right]\right)\right]=-\sum_{j=1}^{N} \rho_{j}\left(\psi\left[\widetilde{\omega}_{j}\right]\right) .
$$


Note that $\widetilde{\omega} \in \pi_{*}\left(\omega_{\mathcal{C} / \mathcal{B}}(* S)\right)$. Hence, the equality (4.16) implies the desired result. To prove (4.16) it is enough to show that for

$$
\begin{aligned}
& \underline{\ell}=\sum_{n=-n_{0}}^{\infty} l_{n} z^{n} \frac{d}{d z} \\
& \omega=A(z) d z=\left(\sum_{m=-m_{0}}^{\infty} a_{m} z^{m}\right) d z
\end{aligned}
$$

we have that

$$
\left[T[\underline{\ell}, \psi[\omega]]=-\psi\left[\left\{\ell(z) A^{\prime}(z)+A(z) \ell^{\prime}(z)\right\} d z\right] .\right.
$$

This can be proved as follows. First note that by (3.2) we have that

$$
\left[L_{n}, \psi_{k+1 / 2}\right]=-(n+k+1) \psi_{n+k+1 / 2} .
$$

Hence

$$
\begin{aligned}
{[T[\underline{\ell}], \psi[\omega]] } & =\sum_{n, k} a_{k} l_{n+1}\left[L_{n}, \psi_{k+1 / 2}\right] \\
& =-\sum_{n, k}(n+k+1) a_{k} l_{n+1} \psi_{n+k+1 / 2} \\
& =-\psi\left[\left\{\ell(z) A^{\prime}(z)+A(z) \ell^{\prime}(z)\right\} d z\right] .
\end{aligned}
$$

From this we conclude that $D(\vec{\ell}) \mathcal{F}_{\text {ab }}^{1}(\mathfrak{F}) \subset \mathcal{F}_{\text {ab }}^{1}(\mathfrak{F})$.

Proposition 4.4. For each element $\vec{\ell} \in \mathcal{L}(\mathfrak{F}), D(\vec{\ell})$ acts on $\mathcal{V}^{\dagger}{ }_{\mathrm{ab}}(\mathfrak{F})$. Moreover, if $\theta(\vec{\ell}) \neq$ 0 , then $D(\vec{\ell})$ acts on $\mathcal{V}^{\dagger}{ }_{\mathrm{ab}}(\mathfrak{F})$ as a twisted first order differential operator.

Proof. Choose

$$
\langle\widetilde{\Psi}| \in \mathcal{V}^{\dagger}{ }_{\mathrm{ab}}(\mathfrak{F}) .
$$

For any element $f \in \pi_{*}\left(\mathcal{O}_{\mathcal{C}}(* S)\right)$ and $|u\rangle \in \mathcal{F}_{N}(\mathcal{B})$, by Proposition 4.3 and (4.14) we have that

$$
\{D(\vec{\ell})\langle\widetilde{\Psi}|\}(\bar{\psi}[f]|u\rangle=\theta(\vec{\ell})(\langle\widetilde{\Psi}|\bar{\psi}[f]| u\rangle)-\langle\widetilde{\Psi}|\{D(\vec{\ell})(\bar{\psi}[f]|u\rangle)\}=0 .
$$

Thus we conclude that

$$
D(\vec{\ell})\langle\widetilde{\Psi}| \in \mathcal{V}^{\dagger}{ }_{\mathrm{ab}}(\mathfrak{F}) .
$$

The remaining statement is an easy consequence of definition (4.13).

Corollary 4.1. The $\mathcal{O}_{\mathcal{B}}$-module $\mathcal{V}^{\dagger}{ }_{a b}(\mathfrak{F})$ is locally free on $\mathcal{B} \backslash D$.

For a proof see [U2], Proposition 4.2.4.

For a coherent $\mathcal{O}_{\mathcal{B}}$-module $\mathcal{G}$, the locus $M$ consisting of points at which $\mathcal{G}$ is not locally free, is a closed analytic subset of $\mathcal{B}$ of codimension at least 2. Therefore, we have the following corollary. 
Corollary 4.2. Let $W$ be the maximal subset of $\mathcal{B}$ over which $\mathcal{V}_{\mathrm{ab}}(\mathfrak{F})$ is not locally free. Then, $W$ is an analytic subset of $\mathcal{B}$ and

$$
W \varsubsetneqq D \text {. }
$$

Since we defined

$$
\mathcal{V}^{\dagger}{ }_{\mathrm{ab}}(\mathfrak{F})=\underline{\operatorname{Hom}}_{\mathcal{O}_{\mathcal{B}}}\left(\mathcal{V}_{\mathrm{ab}}(\mathfrak{F}), \mathcal{O}_{\mathcal{B}}\right)
$$

we have the following corollary.

Corollary 4.3. $\mathcal{V}^{\dagger}$ ab $\left.(\mathfrak{F})\right|_{\mathcal{B} \backslash D}$ is a locally free $\mathcal{O}_{\mathcal{B}}$-module and for any subvariety $Y$ of $\mathcal{B} \backslash D$ we have an $\mathcal{O}_{Y}$-module isomorphism

$$
\mathcal{O}_{Y} \otimes_{\mathcal{O}_{\mathcal{B}}} \mathcal{V}^{\dagger}{ }_{\mathrm{ab}}(\mathfrak{F}) \simeq \mathcal{V}^{\dagger}{ }_{\mathrm{ab}}\left(\left.\mathfrak{F}\right|_{Y}\right)
$$

These two corollaries play a crucial role in proving locally freeness in general. The above corollaries imply the following theorem.

Theorem 4.1. If $\mathfrak{F}$ is a family of $N$-pointed smooth curves with formal coordinates, then $\mathcal{V}_{\mathrm{ab}}(\mathfrak{F})$ and $\mathcal{V}^{\dagger}{ }_{\mathrm{ab}}(\mathfrak{F})$ are locally free $\mathcal{O}_{\mathcal{B}}$-modules and they are dual to each other.

Note that for the bilinear pairing

$$
\langle\cdot \mid \cdot\rangle: \mathcal{V}_{\mathrm{ab}}(\mathfrak{F}) \times \mathcal{V}_{\mathrm{ab}}^{\dagger}(\mathfrak{F}) \rightarrow \mathcal{O}_{\mathcal{B}},
$$

we have the equality

$$
\{D(\vec{\ell})\langle\Psi|\}|u\rangle+\langle\Psi|\{D(\vec{\ell})|u\rangle\}=\theta(\vec{\ell})(\langle\Psi \mid u\rangle) .
$$

Now let us define a connection of $\mathcal{V}^{\dagger}$ ab $(\mathfrak{F})$. First note that by direct calculations we have that

$$
\begin{aligned}
& \left\langle\Phi\left|\rho_{j}\left(\frac{d \psi\left(\zeta_{j}\right)}{d \zeta_{j}} \bar{\psi}\left(\xi_{j}\right)\right)\right| u\right\rangle \\
& =-\sum_{m=-\infty}^{\infty} \sum_{n=1}^{\infty}(m+1)\left\langle\Phi\left|\rho_{j}\left(\psi_{m+1 / 2} \bar{\psi}_{n-1 / 2}\right)\right| u\right\rangle \zeta_{j}^{-m-2} \xi_{j}^{-n} \\
& \quad+\sum_{m=-\infty}^{\infty} \sum_{n=0}^{\infty}(m+1)\left\langle\Phi\left|\rho_{j}\left(\bar{\psi}_{-n-1 / 2} \psi_{m+1 / 2}\right)\right| u\right\rangle \zeta_{j}^{-m-2} \xi_{j}^{n}-\frac{\langle\Phi \mid u\rangle}{\left(\zeta_{j}-\xi_{j}\right)^{2}}
\end{aligned}
$$

This implies

$$
\begin{aligned}
& \left\langle\Phi\left|\rho_{j}\left(T\left(\xi_{j}\right)\right)\right| u\right\rangle\left(d \xi_{j}\right)^{2} \\
& \quad=\lim _{\zeta_{j} \rightarrow \xi_{j}}\left\{\left\langle\Phi\left|\rho_{j}\left(\frac{d \psi\left(\zeta_{j}\right)}{d \zeta_{j}} \bar{\psi}\left(\xi_{j}\right)\right)\right| u\right\rangle d \zeta_{j} d \xi_{j}+\frac{\langle\Phi \mid u\rangle}{\left(\zeta_{j}-\xi_{j}\right)^{2}} d \zeta_{j} d \xi_{j}\right\} .
\end{aligned}
$$

Note that we can as in $[\mathrm{U} 2,3.4]$ define the correlation functions

$$
\left\langle\Phi\left|\frac{d \psi(w)}{d w} \bar{\psi}(z)\right| u\right\rangle d w d z, \quad\langle\Phi|T(z)| u\rangle d z^{2}
$$


such that

$$
\langle\Phi|T(z)| u\rangle d z^{2}=\lim _{w \rightarrow z}\left\{\left\langle\Phi\left|\frac{d \psi(w)}{d w} \bar{\psi}(z)\right| u\right\rangle d w d z+\frac{\langle\Phi \mid u\rangle}{(w-z)^{2}} d w d z\right\} .
$$

Note that $\left\langle\Phi\left|\frac{d \psi(w)}{d w} \bar{\psi}(z)\right| u\right\rangle d w d z$ is a meromorphic section of $p_{1}^{*} \omega_{\mathcal{C} / \mathcal{B}} \otimes_{\mathcal{O}_{\mathcal{C} \times \mathcal{B}}} p_{2}^{*} \omega_{\mathcal{C} / \mathcal{B}}$ over $\mathcal{B}$ whose Laurent expansion in a neighbourhood of $S_{j} \times S_{j}$ is equal to the above expansion of $\left\langle\Phi\left|\rho_{j}\left(\frac{d \psi\left(\zeta_{j}\right)}{d \zeta_{j}} \bar{\psi}\left(\xi_{j}\right)\right)\right| u\right\rangle$, where $p_{i}: \mathcal{C} \times{ }_{\mathcal{B}} \mathcal{C} \rightarrow \mathcal{C}$ is the projection to the $i$-th factor.

Now choose a meromorphic bidifferential

$$
\omega \in H^{0}\left(\mathcal{C} \times{ }_{\mathcal{B}} \mathcal{C}, \omega_{\mathcal{C} \times{ }_{\mathcal{B}} \mathcal{C}}(2 \Delta)\right)
$$

which has the form

$$
\omega(w, z)=\frac{d w d z}{(w-z)^{2}}+\text { holomorphic }
$$

in a neighbourhood of the diagonal $\Delta$ of $\mathcal{C} \times{ }_{\mathcal{B}} \mathcal{C}$. Define $\widetilde{T}(z)$ by

$$
\left\langle\Phi\left|\rho_{j}(\widetilde{T}(z))\right| u\right\rangle(d z)^{2}=\lim _{w \rightarrow z}\left\{\left\langle\Phi\left|\frac{d \psi(w)}{d w} \bar{\psi}(z)\right| u\right\rangle d w d z+\langle\Phi \mid u\rangle \omega(w, z)\right\} .
$$

Then $\left\langle\Phi\left|\rho_{j}(\widetilde{T}(z))\right| u\right\rangle(d z)^{2}$ is a global meromorphic form, that is a meromorphic section of $\omega_{\mathcal{C} / \mathcal{B}}$ over $\mathcal{B}$. The projective connection $S_{\omega}(z)(d z)^{2}$ associated to the bidifferential $\omega$ is defined by

$$
S_{\omega}(z)(d z)^{2}=6 \lim _{w \rightarrow z}\left\{\omega(w, z)-\frac{d w d z}{(w-z)^{2}}\right\} .
$$

Then, by (4.19) we have that

$$
\left.\left\langle\Phi\left|\rho_{j}(T(z))\right| u\right\rangle(d z)^{2}=\langle\Phi| \widetilde{T}(z)\right)|u\rangle(d z)^{2}-\frac{1}{6}\langle\Phi \mid u\rangle S_{\omega}(z)^{2}(d z)^{2} .
$$

Then for any element $|u\rangle \in \mathcal{F}_{N}$ by (4.12)

$$
\begin{array}{r}
\langle\Phi|\{D(\vec{\ell})|u\rangle\}=-\sum_{j=1}^{N} \operatorname{Res}_{\xi_{j}=0}\left(\ell_{j}\left(\xi_{j}\right)\left\langle\Phi\left|\rho_{j}\left(\widetilde{T}\left(\xi_{j}\right)\right)\right| u\right\rangle d \xi_{j}\right) \\
+\frac{1}{6}\langle\Phi \mid u\rangle \sum_{j=1}^{N} \operatorname{Res}_{\xi_{j}=0}\left(\ell_{j}\left(\xi_{j}\right) S_{\omega}\left(\xi_{j}\right) d \xi_{j}\right) .
\end{array}
$$

If $\theta(\vec{\ell})=0$, that is $\vec{\ell}$ is the image of a global vector field $\tau \in \pi_{*}\left(\Theta_{\mathcal{C} / \mathcal{B}}(S)\right)$, then

$$
\ell_{j}\left(\xi_{j}\right)\left\langle\Phi\left|\rho_{j}\left(\widetilde{T}\left(\xi_{j}\right)\right)\right| u\right\rangle d \xi_{j}
$$

is the Laurent expansion of a global meromorphic relative one-form which has poles only at $S_{j}$. Hence the first term of the right hand side of the above equality is zero. Therefore, in this case we have that

$$
\langle\Phi|\{D(\vec{\ell})|u\rangle\}=\frac{1}{6}\langle\Phi \mid u\rangle \sum_{j=1}^{N} \operatorname{Res}_{\xi_{j}=0}\left(\ell_{j}\left(\xi_{j}\right) S_{\omega}\left(\xi_{j}\right) d \xi_{j}\right) .
$$


Now put

$$
b_{\omega}(\vec{\ell})=\sum_{j=1}^{N} \operatorname{Res}_{\xi_{j}=0}\left(\ell_{j}\left(\xi_{j}\right) S_{\omega}\left(\xi_{j}\right) d \xi_{j}\right) .
$$

Then this defines an $\mathcal{O}_{\mathcal{B}}$-module homomorphism

$$
b_{\omega}: \mathcal{L}(\mathfrak{F}) \rightarrow \mathcal{O}_{\mathcal{B}},
$$

and if $\theta(\vec{\ell})=0$ then we have that

$$
\langle\Phi|\{D(\vec{\ell})|u\rangle\}=\frac{1}{6} b_{\omega}(\vec{\ell})\langle\Phi \mid u\rangle .
$$

For a vector field $X \in \Theta(-\log D)$ on $\mathcal{B}$ tangent to $D$ choose $\vec{\ell} \in \mathcal{L}(\mathfrak{F})$ such that $\theta(\vec{\ell})=X$. Then the connection on $\mathcal{V}_{\text {ab }}(\mathfrak{F})$ is defined by

$$
\nabla_{X}^{(\omega)}([|\Psi\rangle])=D(\vec{\ell})([|\Psi\rangle])-\frac{1}{6} b_{\omega}(\vec{\ell})([|\Psi\rangle]),
$$

for $|\Psi\rangle \in \mathcal{V}_{\mathrm{ab}}(\mathfrak{F})$. Dually the connection on $\mathcal{V}^{\dagger} \mathrm{ab}(\mathfrak{F})$ is defined by

$$
\nabla_{X}^{(\omega)}(\langle\Phi|)=D(\vec{\ell})(\langle\Phi|)+\frac{1}{6} b_{\omega}(\vec{\ell})(\langle\Phi|),
$$

for $\langle\Phi| \in \mathcal{V}^{\dagger}{ }_{\mathrm{ab}}(\mathfrak{F})$. These are well-defined, that is the right hand sides of (4.23) and (4.24) are independent of the choice of $\vec{\ell} \in \mathcal{L}(\mathfrak{F})$ with $\theta(\vec{\ell})=X$. Just like for the non-abelian conformal field theory (see for example [U2], section 5) we can prove the following theorem.

Theorem 4.2. The operator $\nabla^{(\omega)}$ defines a projectively flat holomorphic connection of the sheaves $\mathcal{V}_{\mathrm{ab}}(\mathfrak{F})$ and $\mathcal{V}^{\dagger} \mathrm{ab}(\mathfrak{F})$. Moreover, the connection has a regular singularity along the locus $D \subset \mathcal{B}$ which is the locus of the singular curves. The connection $\nabla^{(\omega)}$ depends on the choice of bidifferential $\omega$ and if we choose another bidifferential $\omega^{\prime}$ then there exists a holomorphic one-form $\phi_{\omega, \omega^{\prime}}$ on $\mathcal{B}$ such that

$$
\nabla_{X}^{(\omega)}-\nabla_{X}^{\left(\omega^{\prime}\right)}=\frac{1}{6}\left\langle\phi_{\omega, \omega^{\prime}}, X\right\rangle
$$

Moreover, the curvature form $R$ is given by

$$
R(X, Y)=\frac{1}{6}\left\{b_{\omega}(\vec{n})-X\left(b_{\omega}(\vec{m})\right)+Y\left(b_{\omega}(\vec{\ell})\right)-\sum_{j=1}^{N} \operatorname{Res}_{\xi_{j}=0}\left(\frac{d^{3} \ell_{j}}{d \xi_{j}} m_{j} d \xi_{j}\right)\right\},
$$

where $\left.X, Y \in \Theta_{\mathcal{C} / \mathcal{B}}(* S)\right), \vec{\ell}, \vec{m} \in \mathcal{L}(\mathfrak{F})$ with $X=\theta(\vec{\ell}), Y=\theta(\vec{m})$, and $\vec{n} \in \mathcal{L}(\mathfrak{F})$ is defined by $\vec{n}=[\vec{\ell}, \vec{m}]_{d}($ see (4.11) $)$.

Remark 4.1. For the connection on the sheaf of non-abelian vacua $\mathcal{V}^{\dagger} \vec{\lambda}(\mathfrak{F})$ of level $l$ over $\mathcal{B}$ with gauge symmetry $\mathfrak{g}$, a complex simple Lie algebra, the curvature form $R^{\mathfrak{g}}$ is given by

where

$$
R^{\mathfrak{g}}(X, Y)=\frac{c_{v}}{2} R(X, Y) \cdot \mathrm{id}
$$

$$
c_{v}=\frac{l \cdot \operatorname{dim} \mathfrak{g}}{l+g^{*}}, \quad g^{*} \text { is the dual Coxeter number of } \mathfrak{g} .
$$

For the details see [U2], section 5 . 


\section{Degeneration of Curves and Sewing}

From a curve with one ordinary double point, we construct via the usual sewing a oneparameter family of curves over a disk, whose fibers over the punctured disk are smooth curves. We shall show that the sheaf of $j=0$ ghost vacua gives an invertible sheaf over the whole disk. This implies that the dimension of the space of ghost vacua is one for any pointed curve. The arguments of this section are almost the same, as those from [U2] section 5, we just need to modify a few arguments in our setting.

First we recall certain basic facts about the energy-momentum tensor and the fermion operators. In the $j=0$ ghost system, the energy-momentum tensor $T(z)$ is given by

$$
T^{(0)}(z)=: \frac{d \psi(z)}{d z} \bar{\psi}(z): .
$$

Note that we have the formula

$$
L_{n}=\sum_{k=0}^{\infty} k \bar{\psi}_{n-k+1 / 2} \psi_{k-1 / 2}+\sum_{k=1}^{\infty} k \psi_{-k-1 / 2} \bar{\psi}_{n+k+1 / 2} .
$$

If $n \neq 0$ then by the anti-commutation relation (1.5) we have that $\bar{\psi}_{n-k+1 / 2} \psi_{k-1 / 2}=$ $-\psi_{k-1 / 2} \bar{\psi}_{n-k+1 / 2}$ and $\psi_{-k-1 / 2} \bar{\psi}_{n+k+1 / 2}=-\bar{\psi}_{n+k+1 / 2} \psi_{-k-1 / 2}$. Thus the normal ordering is non-trivial only for $L_{0}^{(0)}$ and we have that

$$
L_{0}^{(0)}=\sum_{\nu>0}(\nu+1 / 2) \bar{\psi}_{-\nu} \psi_{\nu}+\sum_{\nu>0}(\nu-1 / 2) \psi_{-\nu} \bar{\psi}_{\nu} .
$$

In the following, we often use the notation $T(z), L_{n}$ instead of $T^{(0)}(z)$ and $L_{n}^{(0)}$. By (3.2) and (3.3) we have that

$$
\begin{aligned}
& {\left[L_{n}, \psi_{\nu}\right]=-(n+\nu+1 / 2) \psi_{n+\nu},} \\
& {\left[L_{n}, \bar{\psi}_{\nu}\right]=-(n+\nu+1 / 2) \bar{\psi}_{n+\nu}+(n+1) \bar{\psi}_{n+\nu} .}
\end{aligned}
$$

In particular we have that

$$
\left[L_{0}, \psi_{\nu}\right]=-(\nu+1 / 2) \psi_{\nu}, \quad\left[L_{0}, \bar{\psi}_{\nu}\right]=-(\nu-1 / 2) \bar{\psi}_{\nu} .
$$

Note that the degree of the element

$$
|u\rangle=\bar{\psi}_{\mu_{1}} \bar{\psi}_{\mu_{2}} \cdots \bar{\psi}_{\mu_{r}} \psi_{\nu_{s}} \psi_{\nu_{s-1}} \cdots \psi_{\nu_{1}}|0\rangle
$$

with $\mu_{1}<\mu_{2}<\cdots<\mu_{r}<0, \nu_{1}<\nu_{2}<\cdots<\nu_{s}<0$ is given by

$$
d(|u\rangle)=-\sum_{i=1}^{r} \mu_{i}-\sum_{j=1}^{s} \nu_{j}-p^{2} / 2
$$

where $p=r-s$ is the charge of the element. By (5.2) and (5.5) we thus see that

$$
L_{0}|u\rangle=(d+p(p+1) / 2)|u\rangle .
$$

Hence we have the following lemma. 
Lemma 5.1. On $\mathcal{F}_{d}(p)$ the operator $L_{0}$ acts by multiplication by the scalar $d+p(p+1) / 2$.

Lemma 5.2. For any element $|u\rangle \in \mathcal{F}_{d}(p)$ and any half integer $\nu \in \mathbf{Z}_{h}$ we have that

$$
\operatorname{deg}\left(\psi_{\nu}|u\rangle\right)=d+p-(\nu+1 / 2), \quad \operatorname{deg}\left(\bar{\psi}_{\nu}|u\rangle\right)=d-p-(\nu+1 / 2) .
$$

Proof. Note that the charge of $\psi_{\nu}|u\rangle$ is $p-1$ and the charge of $\bar{\psi}_{\nu}|u\rangle$ is $p+1$. By (5.5) we have

$$
L_{0}\left(\psi_{\nu}|u\rangle\right)=\psi_{\nu}\left(L_{0}|u\rangle\right)-(\nu+1 / 2) \psi_{\nu}|u\rangle=\{d+p(p+1) / 2-(\nu+1 / 2)\} \psi_{\nu}|u\rangle .
$$

Hence, we get that

$$
\operatorname{deg}\left(\psi_{\nu}|u\rangle\right)=d+p(p+1) / 2-(\nu+1 / 2)-p(p-1) / 2=d+p-(\nu+1 / 2) .
$$

By the same way we can show that

$$
L_{0}\left(\bar{\psi}_{\nu}|u\rangle\right)=\{d+p(p+1) / 2-(\nu-1 / 2)\} \psi_{\nu}|u\rangle .
$$

Hence

$$
\operatorname{deg}\left(\bar{\psi}_{\nu}|u\rangle\right)=d-p-(\nu+1 / 2)
$$

Let

$$
\begin{aligned}
& |u\rangle=\bar{\psi}_{\mu_{1}} \bar{\psi}_{\mu_{2}} \cdots \bar{\psi}_{\mu_{r}} \psi_{\nu_{s}} \psi_{\nu_{s-1}} \cdots \psi_{\nu_{1}}|0\rangle \\
& \mu_{1}<\mu_{2}<\cdots<\mu_{r}<0, \quad \nu_{1}<\nu_{2}<\cdots<\nu_{s}<0
\end{aligned}
$$

and $M \in \mathcal{M}(p)$ be the Maya diagram corresponding to $|u\rangle$. Then

$$
|M\rangle=(-1)^{\sum_{i=1}^{s} \nu_{i}+s / 2} \bar{\psi}_{\mu_{1}} \bar{\psi}_{\mu_{2}} \cdots \bar{\psi}_{\mu_{r}} \psi_{\nu_{s}} \psi_{\nu_{s-1}} \cdots \psi_{\nu_{1}}|0\rangle .
$$

Let us introduce a bilinear pairing

$$
(\quad): \mathcal{F}(p) \times \mathcal{F}(p) \rightarrow \mathbf{C}
$$

by putting

$$
(|M\rangle|| N\rangle)=\langle M \mid N\rangle=\delta_{M, N}
$$

for Maya diagrams $M, N \in \mathcal{M}(p)$. The pairing is perfect on $\mathcal{F}_{d}(p) \times \mathcal{F}_{d}(p)$ and zero on $\mathcal{F}_{d}(p) \times \mathcal{F}_{d^{\prime}}(p)$ for $d \neq d^{\prime}$. Note that the bilinear pairing $(\mid)$ is symmetric.

\section{Lemma 5.3.}

$$
\begin{aligned}
\left.\left.\left(\psi_{\nu}|u\rangle|| v\right\rangle\right)=\left(|u\rangle\left|\bar{\psi}_{-\nu}\right| v\right\rangle\right), & \text { for }|u\rangle \in \mathcal{F}(p+1),|v\rangle \in \mathcal{F}(p), \\
\left.\left.\left(\bar{\psi}_{\nu}|u\rangle|| v\right\rangle\right)=\left(|u\rangle\left|\psi_{-\nu}\right| v\right\rangle\right), & \text { for }|u\rangle \in \mathcal{F}(p-1),|v\rangle \in \mathcal{F}(p) .
\end{aligned}
$$


Proof. It is enough to consider the case in which $|u\rangle$ and $|v\rangle$ are Maya diagrams. Assume

$$
|M\rangle=(-1)^{\sum_{i=1}^{s} \nu_{i}+s / 2} \bar{\psi}_{\mu_{1}} \bar{\psi}_{\mu_{2}} \cdots \bar{\psi}_{\mu_{r}} \psi_{\nu_{s}} \psi_{\nu_{s-1}} \cdots \psi_{\nu_{1}}|0\rangle,
$$

with $\mu_{1}<\mu_{2}<\cdots<\mu_{r}<0, \nu_{1}<\nu_{2}<\cdots<\nu_{s}<0$. Then we have that

$$
\psi_{\nu}|M\rangle=(-1)^{\sum_{i=1}^{s} \nu_{i}+s / 2+r} \bar{\psi}_{\mu_{1}} \bar{\psi}_{\mu_{2}} \cdots \bar{\psi}_{\mu_{r}} \psi_{\nu} \psi_{\nu_{s}} \psi_{\nu_{s-1}} \cdots \psi_{\nu_{1}}|0\rangle .
$$

For simplicity assume that $\nu>\nu_{s}$. Then $\left.\left(\psi_{\nu}|M\rangle|| N\right\rangle\right) \neq 0$ if and only if the Maya diagram $N$ has the form

$$
|N\rangle=(-1)^{\sum_{i=1}^{s} \nu_{i}+\nu+s / 2+1 / 2} \bar{\psi}_{\mu_{1}} \bar{\psi}_{\mu_{2}} \cdots \bar{\psi}_{\mu_{r}} \psi_{\nu} \psi_{\nu_{s}} \psi_{\nu_{s-1}} \cdots \psi_{\nu_{1}}|0\rangle .
$$

In this case

$$
\left.\left(\psi_{\nu}|M\rangle|| N\right\rangle\right)=(-1)^{\nu+1 / 2+r}
$$

On the other hand

$$
\bar{\psi}_{-\nu}|N\rangle=(-1)^{\nu+1 / 2+r}|M\rangle \text {. }
$$

Hence

$$
\left.\left.\left(\psi_{\nu}|M\rangle|| N\right\rangle\right)=\left(|M\rangle\left|\bar{\psi}_{-\nu}\right| N\right\rangle\right) .
$$

Other cases can be treated similarly.

Let $r: \mathcal{F}(p) \rightarrow \mathcal{F}(-p)$ be a mapping defined by taking the mirror image of the Maya diagram with respect to 0 and interchange white and black. Namely, if

$$
|M\rangle=(-1)^{\sum_{i=1}^{s} \nu_{i}+s / 2} \bar{\psi}_{\mu_{1}} \bar{\psi}_{\mu_{2}} \cdots \bar{\psi}_{\mu_{r}} \psi_{\nu_{s}} \psi_{\nu_{s-1}} \cdots \psi_{\nu_{1}}|0\rangle,
$$

with $\mu_{1}<\mu_{2}<\cdots<\mu_{r}<0, \nu_{1}<\nu_{2}<\cdots<\nu_{s}<0$, then $r(M)$ is defined by

$$
|r(M)\rangle=(-1)^{\sum_{j=1}^{r} \mu_{j}+r / 2} \bar{\psi}_{\nu_{1}} \bar{\psi}_{\nu_{2}} \cdots \bar{\psi}_{\nu_{s}} \psi_{\mu_{r}} \psi_{\mu_{r-1}} \cdots \psi_{\mu_{1}}|0\rangle .
$$

Note that we have

$$
\operatorname{charge}(r(M))=-\operatorname{charge}(M) \text { and } \operatorname{deg}(r(M))=\operatorname{deg}(M) .
$$

Hence, we have a linear map

$$
r: \mathcal{F}_{d}(p) \rightarrow \mathcal{F}_{d}(-p)
$$

Now for any integer $p$ let us introduce a bilinear pairing

$$
\{\mid\}: \mathcal{F}_{d}(p) \times \mathcal{F}_{d}(-p) \rightarrow \mathbf{C} .
$$

For that purpose put

$$
\alpha(n)=\left\{\begin{array}{ccc}
1, & n \equiv 1,2 \quad(\bmod 4) \\
-1, & n \equiv 0,3 \quad(\bmod 4)
\end{array}\right.
$$

Then it is easy to show that we for any $n \in \mathbf{Z}$ get that

$$
\alpha(n+1)=(-1)^{n+1} \alpha(n) .
$$


The bilinear pairing $\{\mid\}$ is defined by

$$
\{|u\rangle|| v\rangle\}=\alpha(\operatorname{charge}(|u\rangle))(|u\rangle \mid r(|v\rangle)) .
$$

In particular for Maya diagrams $M \in \mathcal{M}(p)$ and $N \in \mathcal{M}(-p)$ we have that

$$
\{|M\rangle|| N\rangle\}=\alpha(p)\langle M \mid r(N)\rangle=\alpha(p) \delta_{M, r(N)} .
$$

Note that the pairing $\{\mid\}: \mathcal{F}_{d}(p) \times \mathcal{F}_{d}(-p) \rightarrow \mathbf{C}$ is perfect and that it can be extended to $\mathcal{F}(p) \times \mathcal{F}(-p) \rightarrow \mathbf{C}$ by requiring that the pairing is zero on $\mathcal{F}_{d}(p) \times \mathcal{F}_{d^{\prime}}(-p)$ for $d \neq d^{\prime}$. Now from the above Lemma 5.3 we obtain the following results.

\section{Lemma 5.4.}

$$
\begin{aligned}
& \left.\left.\left\{\psi_{\nu}|u\rangle|| v\right\rangle\right\}=(-1)^{-\nu-1 / 2}\left\{|u\rangle\left|\psi_{-\nu}\right| v\right\rangle\right\} \\
& \left.\left.\left\{\bar{\psi}_{\nu}|u\rangle|| v\right\rangle\right\}=(-1)^{-\nu-1 / 2}\left\{|u\rangle\left|\bar{\psi}_{-\nu}\right| v\right\rangle\right\}
\end{aligned}
$$

Proof. By the definition of the mapping $r$ we have that

$$
\begin{aligned}
\psi_{\nu} r(|v\rangle) & =(-1)^{-\nu+1 / 2+\operatorname{charge}(|v\rangle)} r\left(\bar{\psi}_{-\nu}|v\rangle\right), \\
\bar{\psi}_{-\nu} r(|v\rangle) & =(-1)^{-\nu+1 / 2+\operatorname{charge}(|v\rangle)} r\left(\psi_{-\nu}|v\rangle\right) .
\end{aligned}
$$

Hence by Lemma 5.3 and (5.7) for $|u\rangle \in \mathcal{F}(p+1)$ and $|v\rangle \in \mathcal{F}(-p)$ we see that

$$
\begin{aligned}
\left.\left\{\psi_{\nu}|u\rangle|| v\right\rangle\right\} & =\alpha(p)\left(\psi_{\nu}|u\rangle \mid r(|v\rangle)\right)=\alpha(p)\left(|u\rangle \mid \bar{\psi}_{-\nu} r(|v\rangle)\right) \\
& =(-1)^{-\nu+1 / 2+p} \alpha(p)\left(|u\rangle \mid r\left(\psi_{-\nu}|v\rangle\right)\right) \\
& =(-1)^{-\nu-1 / 2} \alpha(p+1)\left(|u\rangle \mid r\left(\psi_{-\nu}|v\rangle\right)\right) \\
& \left.=(-1)^{-\nu-1 / 2}\left\{|u\rangle\left|\psi_{-\nu}\right| v\right\rangle\right\} .
\end{aligned}
$$

By a similar argument we can show the second equality.

QED

Let us introduce the shift operator $s: \mathcal{F}(p) \rightarrow \mathcal{F}(p+1)$ by defining

$$
s(\mu)(\nu)=\mu(\nu-1)+1,
$$

for a Maya diagram $M$ with characteristic function $\mu$. E.g.

$$
\begin{aligned}
& s\left(\bar{\psi}_{\mu_{1}} \bar{\psi}_{\mu_{2}} \cdots \bar{\psi}_{\mu_{r}} \psi_{\nu_{s}} \psi_{\nu_{s-1}} \cdots \psi_{\nu_{1}}|0\rangle\right) \\
& =\bar{\psi}_{\mu_{1}-1} \bar{\psi}_{\mu_{2}-1} \cdots \bar{\psi}_{\mu_{r}-1} \psi_{\nu_{s}+1} \psi_{\nu_{s-1}+1} \cdots \psi_{\nu_{1}+1}|1\rangle \\
& =(-1)^{s} \bar{\psi}_{\mu_{1}-1} \bar{\psi}_{\mu_{2}-1} \cdots \bar{\psi}_{\mu_{r}-1} \bar{\psi}_{-1 / 2} \psi_{\nu_{s}+1} \psi_{\nu_{s-1}+1} \cdots \psi_{\nu_{1}+1}|0\rangle \\
& \quad+\delta_{\nu_{s},-\frac{1}{2}} \bar{\psi}_{\mu_{1}-1} \bar{\psi}_{\mu_{2}-1} \cdots \bar{\psi}_{\mu_{r}-1} \psi_{\nu_{s-1}+1} \cdots \psi_{\nu_{1}+1}|0\rangle
\end{aligned}
$$

with $\mu_{1}<\mu_{2}<\cdots<\mu_{r}<0, \nu_{1}<\nu_{2}<\cdots<\nu_{s}<0$.

Now the bilinear pairings

$$
\{\mid\}_{+}: \mathcal{F}(p) \times \mathcal{F}(-p-1) \rightarrow \mathbf{C}
$$

is defined by

$$
\{|u\rangle|| v\rangle\}_{+}=\{|u\rangle \mid s(|v\rangle)\}=\alpha(\operatorname{charge}(|u\rangle))(|u\rangle, \mid r(s(|v\rangle)) .
$$

Note that the pairing $\{\mid\}_{+}$is perfect on $\mathcal{F}_{d}(p) \times \mathcal{F}_{d}(-p-1)$ and zero on $\mathcal{F}_{d}(p) \times$ $\mathcal{F}_{d^{\prime}}(-p-1)$ for $d \neq d^{\prime}$.

Finally we obtain the following result which play an important role in the sewing procedure. 
Lemma 5.5. We have the following equalities

$$
\begin{aligned}
& \left.\left.\left\{\psi_{\nu}|u\rangle|| v\right\rangle\right\}_{+}=(-1)^{-\nu-1 / 2}\left\{|u\rangle\left|\psi_{-\nu-1}\right| v\right\rangle\right\}_{+}, \\
& \left.\left.\left\{\bar{\psi}_{\nu}|u\rangle|| v\right\rangle\right\}_{+}=(-1)^{-\nu-1 / 2}\left\{|u\rangle\left|\bar{\psi}_{-\nu+1}\right| v\right\rangle\right\}_{+} .
\end{aligned}
$$

Proof. These results follow from Lemma 5.4 and (5.9).

Let us now consider a one-parameter family of curves which is a family of smooth curves degenerating to a single nodal curve.

Let $C_{0}$ be a complete curve with only one ordinary double point $P$ such that $C_{0} \backslash\{P\}$ is non-singular. Let $Q_{1}, Q_{2}, \ldots, Q_{N}$ be distinct non-singular points on $C_{0}$. Let $\nu: \widetilde{C}_{0} \rightarrow C_{0}$ be the normalization of the singular curve. Put $\left\{P_{+}, P_{-}\right\}=\nu^{-1}(P)$.

Lemma 5.6. There exist a meromorphic vector field $\tilde{l} \in H^{0}\left(\widetilde{C}_{0}, \Theta_{C_{0}}\left(* \sum_{j=1}^{N} Q_{j}\right)\right)$ and a coordinate neighbourhood $U_{+}$(resp. $U_{-}$) of the point $P_{+}$(resp. $\left.P_{-}\right)$with local coordinate $z$ with center $P_{+}\left(r e s p . w\right.$ with center $\left.P_{-}\right)$such that we have

$$
\begin{array}{rlrl}
U_{+}=\{z|| z \mid<1\}, & & U_{-}=\{w|| w \mid<1\} \\
\left.\tilde{l}\right|_{U_{+}}=\frac{1}{2} z \frac{d}{d z}, & \tilde{l}_{U_{-}}=\frac{1}{2} w \frac{d}{d w} .
\end{array}
$$

This lemma is a special case of lemma 5.3.1 in [U2]. For the convenience of the read we give the proof in this special case here.

Proof. Choose coordinate neighbourhood $U_{+}$(resp. $U_{-}$) and a local coordinate $x$ with center $P_{+}\left(y\right.$ with center $\left.P_{-}\right)$. Then we can find a meromorphic vector field $\tilde{l} \in H^{0}\left(\widetilde{C}_{0}, \Theta_{C_{0}}\left(* \sum_{j=1}^{N} Q_{j}\right)\right)$ such that

$$
\tilde{l}_{U_{+}}=\left(\frac{1}{2} x+a_{2} x^{2}+\cdots\right) \frac{d}{d x}, \quad \tilde{l}_{U_{-}}=\left(\frac{1}{2} y+b_{2} y^{2}+\cdots\right) \frac{d}{d y} .
$$

Then, by solving the following differential equations

$$
\begin{aligned}
\tilde{l}(z) & =\frac{1}{2} z \frac{d z}{d x}, \quad z(0)=0, \\
\tilde{l}(w) & =\frac{1}{2} w \frac{d w}{d y}, \quad w(0)=0,
\end{aligned}
$$

we obtain holomorphic functions $z$ and $w$ in neighbourhoods of $P_{+}$and $P_{-}$, respectively. It is easy to show that $z$ and $w$ give local coordinates with center $P_{+}$and $P_{-}$, respectively. Moreover, choosing $U_{ \pm}$smaller if necessary, by the differential equations we have

$$
\tilde{l}_{U_{+}}=\frac{1}{2} z \frac{d}{d z}, \quad \tilde{l}_{U_{-}}=\frac{1}{2} w \frac{d}{d w} .
$$

Replacing $z$ by $c z$ with $c \neq 0$ and $w$ by $c^{\prime} w$ with $c^{\prime} \neq 0$ we may assume that

$$
U_{+}=\{z|| z \mid<1\}, \quad U_{-}=\{w|| w \mid<1\}
$$


Let us now construct a flat one-parameter deformation of $C_{0}$. We do this just like in section 5.3 in [U2]. Let

$$
\begin{aligned}
D & =\{q \in \mathbf{C}|| q \mid<1\} \\
S & =\left\{(x, y, q) \in \mathbf{C}^{3}|x y=q,| x|<1,| y|<1,| q \mid<1\right\} \\
Z & =\left\{(P, q) \in \widetilde{C}_{0} \times D \mid P \in \widetilde{C}_{0} \backslash\left(U_{+} \cup U_{-}\right), \text {or } P \in U_{+},|z(P)|>|q|\right\} \\
W & =\left\{(Q, q) \in \widetilde{C}_{0} \times D \mid Q \in \widetilde{C}_{0} \backslash\left(U_{+} \cup U_{-}\right), \text {or } Q \in U_{-},|w(Q)|>|q|\right\}
\end{aligned}
$$

Now introduce an equivalence relation $\sim$ on $Z \sqcup S \sqcup W$ as follows.

$$
\begin{array}{ll}
\text { 1) } & Z \cap\left(U_{+} \times D\right) \ni(P, q) \sim\left(x, y, q^{\prime}\right) \in S \Longleftrightarrow\left(x, y, q^{\prime}\right)=(z(P), q / z(P), q) \\
2) & W \cap\left(U_{-} \times D\right) \ni(Q, q) \sim\left(x, y, q^{\prime}\right) \in S \Longleftrightarrow\left(x, y, q^{\prime}\right)=(q / w(P), w(P), q) \\
2) & Z \ni(P, q) \sim\left(Q, q^{\prime}\right) \in W \Longleftrightarrow(P, q)=\left(Q, q^{\prime}\right)
\end{array}
$$

Let $\mathcal{C}$ be the two-dimensional complex manifold obtained as the quotient of $Z \sqcup S \sqcup W$ by this equivalence relation. There is a proper holomorphic map $\pi: \mathcal{C} \rightarrow D$ such that the fiber over the origin is $C_{0}$ and for $q \neq 0$ the fiber $C_{q}=\pi^{-1}(q)$ is a non-singular curve of genus $g+1$ or $g$ according as $\widetilde{C}_{0}$ is connected or not, where the genus of $\widetilde{C}_{0}$ is $g$. The family $\pi: \mathcal{C} \rightarrow D$ is a flat deformation (or smoothing) of $C_{0}$. Let us assume that $Q_{j} \notin U_{+} \cup U_{-}$. Then, the point $Q_{j} \in C_{0} \backslash\{P\}$ defines a holomorphic section $\sigma_{i}: D \rightarrow \mathcal{C}$. Let us choose a local coordinate $\xi_{j}$ with center $Q_{i}$. Put $\mathfrak{F}=\left(\pi: \mathcal{C} \rightarrow D ; \sigma_{1}, \ldots, \sigma_{N} ; \xi_{1}, \ldots, \xi_{N}\right)$. The meromorphic vector field $\tilde{l}$ given in Lemma 5.6 defines local vector fields

$$
\vec{l}=\left(l_{1}\left(\xi_{1}\right) \frac{d}{d \xi_{1}}, l_{2}\left(\xi_{2}\right) \frac{d}{d \xi_{2}}, \ldots, l_{N}\left(\xi_{N}\right) \frac{d}{d \xi_{N}}\right)
$$

where $l_{j}\left(\xi_{j}\right) \frac{d}{d \xi_{j}}$ is the Laurent expansion of $\tilde{l}$ with respect to the coordinate $\xi_{j}$ with center $Q_{j}$

Lemma 5.7. The local vector fields $\vec{l}$ is an element of $\mathcal{L}(\mathfrak{F})$ and we have

$$
\theta(\vec{l})=q \frac{d}{d q}
$$

Proof. The meromorphic vector field

$$
\tilde{l}-q \frac{q}{d q}
$$

is a holomorphic section of $\pi_{*} \Theta_{\mathcal{C}}^{\prime}\left(* \sum_{j=1}^{N} \sigma_{j}(D)\right)_{\pi}$ over $D$. This show the first part of the proposition. A proof of the second part can be found in [U2, Corollary 5.3.3 ].

QED

Theorem 5.1. Put

$$
\widetilde{\mathfrak{X}}=\left(\widetilde{C}_{0} ; P_{+}, P_{-}, Q_{1}, \ldots, Q_{N}, z, w, \xi_{1}, \ldots, \xi_{N}\right)
$$

and

$$
\mathfrak{X}=\left(C_{0} ; Q_{1}, \ldots, Q_{N}, \xi_{1}, \ldots, \xi_{N}\right) .
$$


Then for any element $\langle\Phi| \in \mathcal{V}^{\dagger}{ }_{\mathrm{ab}}(\widetilde{\mathfrak{X}})$ there exists a unique global flat section $\langle\widetilde{\Phi}(q)|$ of the sheaf of ghost vacua $\mathcal{V}^{\dagger}{ }_{\mathrm{ab}}(\mathfrak{F})$ over the disk such that $\langle\widetilde{\Phi}(0)|$ is the element of $\mathcal{V}^{\dagger}{ }_{\mathrm{ab}}(\mathfrak{X})$ corresponding to the element $\langle\Phi|$, i.e.

$$
\langle\widetilde{\Phi}(0) \mid u\rangle=\left\langle\Phi \mid 0_{+,-} \otimes u\right\rangle
$$

for any $|u\rangle \in \mathcal{F}_{N}$.

Proof. Let $\left\{v_{i}(d, p)\right\}_{i=1, \ldots, m_{d}}$ be a basis of $\mathcal{F}_{d}(p)$ for any $p \in \mathbf{Z}$ and $\left\{v^{i}(d, p)\right\}_{i=1, \ldots, m_{d}}$ be the dual basis of $\mathcal{F}_{d}(-p-1)$ with respect to the pairing $\{\mid\}_{+}$. Note that $\mathcal{F}_{0}(p)$ is spanned by $|p\rangle$ over $\mathbf{C}$ and we choose $v_{1}(0, p)=|p\rangle$ for all $p \in \mathbf{Z}$. Then $v^{1}(0,-p-1)=$ $\alpha(p)|-p-1\rangle$.

Define $\langle\widetilde{\Phi}(q)|$ by

$$
\langle\widetilde{\Phi}(q) \mid u\rangle=\sum_{p \in \mathbf{Z}}\left\{\sum_{d=0}^{\infty} \sum_{i=1}^{m_{d}}(-1)^{p+d}\left\langle\Phi \mid v_{i}(d, p) \otimes v^{i}(d,-p-1) \otimes u\right\rangle\right\} q^{d+p(p+1) / 2} .
$$

Then by Theorem $3.5\langle\widetilde{\Phi}(0)| \in \mathcal{V}^{\dagger}{ }_{\mathrm{ab}}(\mathfrak{X})$ is the element corresponding to $\langle\Phi|$.

Let us show that $\langle\widetilde{\Phi}(q)|$ satisfies the gauge conditions. Since $\langle\widetilde{\Phi}(q)|$ is defined as a formal power series, we will first prove the formal gauge conditions. Later we shall show that $\langle\widetilde{\Phi}(q)|$ satisfies a differential equation of Fuchsian type so that it converges. Then, the formal gauge conditions implies the usual gauge conditions.

Choose $\tau \in \pi_{*}\left(\omega_{\mathcal{C} / \mathcal{B}}(* S)\right)$. In a neighbourhood of the double point $P$ in $\mathcal{C}, \tau$ can be expressed in the form

$$
\tau=\left(\sum_{n, m \geq 0} a_{n, m} z^{n} w^{m}\right)[d z \wedge d w / d q]
$$

where $[d z \wedge d w / d q]$ is a local basis of $\omega_{\mathcal{C} / \mathcal{B}}$ such that

$$
\left.\nu^{*}([d z \wedge d w / d q])\right|_{U_{+}}=-\frac{d z}{z},\left.\quad \nu^{*}([d z \wedge d w / d q])\right|_{U_{-}}=\frac{d w}{w} .
$$

Now put

$$
\begin{aligned}
& \tau_{+}=\left(\sum_{n, m \geq 0} a_{n, m} z^{n}\left(\frac{q}{z}\right)^{m}\right)\left(-\frac{d z}{z}\right)=\sum_{n=0}^{\infty}\left\{-\sum_{m=0}^{\infty} a_{m, n} z^{m-n-1} d z\right\} q^{n} \\
& \left.\tau_{-}=\left(\sum_{n, m \geq 0} a_{n, m}\left(\frac{q}{w}\right)^{n} w^{m}\right) \frac{d w}{w}=\sum_{n=0}^{\infty}\left\{\sum_{m=0}^{\infty} a_{n, m} w^{m-n-1} d w\right)\right\} q^{n}
\end{aligned}
$$

and

$$
\begin{aligned}
\tau_{+}^{(n)} & =-\left(\sum_{m=0}^{\infty} a_{m, n} z^{m-n-1}\right) d z \\
\tau_{-}^{(n)} & =\left(\sum_{m=0}^{\infty} a_{n, m} w^{m-n-1}\right) d w .
\end{aligned}
$$

Then

$$
\tau_{+}=\sum_{n=0}^{\infty} \tau_{+}^{(n)} q^{n}, \quad \tau_{-}=\sum_{n=0}^{\infty} \tau_{-}^{(n)} q^{n}
$$


It is easy to show that there is a unique $\tau^{(n)} \in H^{0}\left(\widetilde{C}_{0}, \omega_{\widetilde{C}_{0}}\left(*\left(P_{+}+P_{-}+\sum_{j=1}^{N} Q_{j}\right)\right)\right.$ such that $\left\{\tau_{ \pm}^{(n)}\right.$ is the Taylor expansion of $\tau^{(n)}$ at $P_{ \pm}$. the data $\left\{\tau_{+}^{(n)}, \tau_{-}^{(n)}, \tau^{(n)}(\xi)\right\}$ define a meromorphic one-form. Hence by the first gauge condition for $\langle\Phi|$ we see that

$$
\begin{aligned}
& \left\langle\Phi \mid \psi\left[\tau_{+}^{(n)}\right] v_{i}(d, p) \otimes v^{i}(d,-p-1) \otimes u\right\rangle \\
& \quad+(-1)^{p}\left\langle\Phi \mid v_{i}(d, p) \otimes \psi\left[\tau_{-}^{(n)}\right] v^{i}(d,-p-1) \otimes u\right\rangle \\
& \quad-\sum_{j=1}^{N}\left\langle\Phi \mid v_{i}(d, p) \otimes v^{i}(d,-p-1) \otimes \rho_{j}\left(\psi\left[\tau_{j}^{(n)}\right]\right) u\right\rangle=0 .
\end{aligned}
$$

Hence

$$
\begin{aligned}
& \sum_{j=1}^{N}\left\langle\widetilde{\Phi}(q) \mid \rho_{j}\left(\psi\left[\sum_{n=0}^{\infty} \tau_{j}^{(n)} q^{n}\right]\right) u\right\rangle=\sum_{j=1}^{N} \sum_{n=0}^{\infty}\left\langle\widetilde{\Phi}(q) \mid \rho_{j}\left(\psi\left[\tau_{j}^{(n)}\right]\right) u\right\rangle q^{n} \\
& =\sum_{p \in \mathbf{Z}} \sum_{n=0}^{\infty} \sum_{d=0}^{\infty} \sum_{i=1}^{m_{d}}(-1)^{p+d}\left\{\left\langle\Phi \mid \psi\left[\tau_{+}^{(n)}\right] v_{i}(d, p) \otimes v^{i}(d,-p-1) \otimes u\right\rangle\right. \\
& \left.+(-1)^{p}\left\langle\Phi \mid v_{i}(d, p) \otimes \psi\left[\tau_{-}^{(n)}\right] v^{i}(d,-p-1) \otimes u\right\rangle\right\} q^{d+n+p(p+1) / 2}
\end{aligned}
$$

Note that

$$
\begin{aligned}
& \psi\left[\tau_{+}^{(n)}\right]=-\sum_{m=0}^{\infty} a_{m, n} \psi_{m-n-1 / 2}, \\
& \psi\left[\tau_{-}^{(n)}\right]=\sum_{m=0}^{\infty} a_{n, m} \psi_{m-n-1 / 2} .
\end{aligned}
$$

Then by Lemma 5.5

$$
\begin{aligned}
& \sum_{i=1}^{m_{d}}\left\langle\Phi \mid \psi\left[\tau_{+}^{(n)}\right] v_{i}(d, p) \otimes v^{i}(d,-p-1) \otimes u\right\rangle q^{d+n+p(p+1) / 2} \\
=- & \sum_{i=1}^{m_{d}} \sum_{m=0}^{\infty} a_{m, n}\left\langle\Phi \mid \psi_{m-n-1 / 2} v_{i}(d, p) \otimes v^{i}(d,-p-1) \otimes u\right\rangle q^{d+n+p(p+1) / 2} \\
=- & \sum_{m=0}^{\infty} \sum_{i=1}^{m_{d}} \sum_{j=1}^{m_{d+p-m+n}} a_{m, n}\left\{\psi_{m-n-1 / 2} v_{i}(d, p) \mid v^{j}(d+p-m+n,-p)\right\}_{+} \\
& \cdot\left\langle\Phi \mid v_{j}(d+p-m+n, p-1) \otimes v^{i}(d,-p-1) \otimes u\right\rangle q^{d+n+p(p+1) / 2} \\
=- & \sum_{m=0}^{\infty} \sum_{i=1}^{m_{d}} \sum_{j=1}^{m_{d+p-m+n}}(-1)^{n-m} a_{m, n}\left\{v_{i}(d, p) \mid \psi_{n-m-1 / 2} v^{j}(d+p-m+n,-p)\right\}_{+} \\
& \cdot\left\langle\Phi \mid v_{j}(d+p-m+n, p-1) \otimes v^{i}(d,-p-1) \otimes u\right\rangle q^{d+n+p(p+1) / 2} \\
= & \sum_{m=0}^{\infty} \sum_{j=1}^{m_{d+p-m+n}}(-1)^{n-m+1} a_{m, n}\langle\Phi| v_{j}(d+p-m+n, p-1)
\end{aligned}
$$

$\left.\otimes \psi_{n-m-1 / 2} v^{j}(d+p-m+n,-p) \otimes u\right\rangle q^{d+n+p(p+1) / 2}$. 
Hence

$$
\begin{aligned}
& \sum_{n=0}^{\infty} \sum_{i=1}^{m_{d}}(-1)^{d+p}\left\langle\Phi \mid \psi\left[\tau_{+}^{(n)}\right] v_{i}(d, p) \otimes v^{i}(d,-p-1) \otimes u\right\rangle q^{d+n+p(p+1) / 2} \\
& =\sum_{n=0}^{\infty} \sum_{m=0}^{\infty} \sum_{j=1}^{m_{d+p-n+m}}(-1)^{d-n+m+p+1} a_{n, m}\langle\Phi| v_{j}(d+p-n+m, p-1) \\
& \left.\otimes \psi_{m-n-1 / 2} v^{j}(d+p-n+m,-p) \otimes u\right\rangle q^{d+m+p(p+1) / 2} .
\end{aligned}
$$

Put

$$
\widetilde{d}=d+p-n+m \text {. }
$$

Then

$$
\widetilde{d}+n+p(p-1) / 2=d+m+p(p+1) / 2 .
$$

Hence the right hand side of the last equality can be rewritten in the form

$$
\begin{aligned}
& \sum_{n=0}^{\infty} \sum_{m=0}^{\infty} \sum_{j=1}^{m_{\widetilde{d}}}(-1)^{\widetilde{d}+1} a_{n, m}\left\langle\Phi \mid v_{j}(\widetilde{d}, p-1) \otimes \psi_{m-n-1 / 2} v^{j}(\widetilde{d},-p) \otimes u\right\rangle q^{\widetilde{d}+n+p(p-1) / 2} \\
& =\sum_{n=0}^{\infty} \sum_{j=1}^{m_{\widetilde{d}}}(-1)^{\widetilde{d}+1}\left\langle\Phi \mid v_{j}(\widetilde{d}, p-1) \otimes \psi\left[\tau_{-}^{(n)}\right] v^{j}(\widetilde{d},-p) \otimes u\right\rangle q^{\widetilde{d}+n+p(p-1) / 2} .
\end{aligned}
$$

Hence

$$
\begin{aligned}
& \sum_{p \in \mathbf{Z}} \sum_{n=0}^{\infty} \sum_{d=0}^{\infty} \sum_{i=1}^{m_{d}}(-1)^{p+d}\left\langle\Phi \mid \psi\left[\tau_{+}^{(n)}\right] v_{i}(d, p) \otimes v^{i}(d,-p-1) \otimes u\right\rangle q^{d+n+p(p+1) / 2} \\
& =-\sum_{p \in \mathbf{Z}} \sum_{n=0}^{\infty} \sum_{d=0}^{\infty} \sum_{i=1}^{m_{d}}(-1)^{d}\left\langle\Phi \mid v_{j}(d, p-1) \otimes \psi\left[\tau_{-}^{(n)}\right] v^{j}(d,-p) \otimes u\right\rangle q^{d+n+p(p-1) / 2} \\
& =-\sum_{p \in \mathbf{Z}} \sum_{n=0}^{\infty} \sum_{d=0}^{\infty} \sum_{i=1}^{m_{d}}(-1)^{d}\left\langle\Phi \mid v_{j}(d, p) \otimes \psi\left[\tau_{-}^{(n)}\right] v^{j}(d,-p-1) \otimes u\right\rangle q^{d+n+p(p-1) / 2}
\end{aligned}
$$

Therefore, by (5.11) we conclude that

$$
\sum_{j=1}^{N}\left\langle\widetilde{\Phi}(q) \mid \rho_{j}\left(\psi\left[\sum_{n=0}^{\infty} \tau_{j}^{(n)} q^{n}\right]\right) u\right\rangle=0 .
$$

This proves the first formal gauge condition. Similarly, we can show that the second formal gauge condition also holds.

Next we shall show that the formal power series (5.10) is a formal solution of a Fuchsian differential equation. Hence the power series (5.10) indeed converges. Therefore the formal gauge conditions are nothing but the usual gauge conditions.

Note that on the punctured disk $D^{*}$ we have the connection introduced in section 4 . For the local vector field $\vec{l}$ defined by the meromorphic vector field $\widetilde{l}$ in Lemma 5.6 the corresponding connection has the form

$$
q \frac{d}{d q}(\langle\widetilde{\Phi}(q) \mid u\rangle)-\sum_{j=1}^{N}\left\langle\widetilde{\Phi}(q) \mid \rho_{j}\left(T\left[\underline{l}_{j}\right]\right) u\right\rangle-\frac{1}{6} b_{\omega}(\vec{l})\langle\widetilde{\Phi}(q) \mid u\rangle=0
$$


where $\omega$ is an element of

$$
H^{0}\left(\mathcal{C} \times{ }_{D} \mathcal{C}, \omega_{\mathcal{C} / D}^{\otimes 2}(2 \Delta)\right)
$$

with

$$
\omega=\frac{d v d u}{(v-u)^{2}}+\text { holomorphic at } \Delta,
$$

where $\Delta$ is the diagonal of $\mathcal{C} \times{ }_{D} \mathcal{C}$ and if $p_{j}: \mathcal{C} \times{ }_{D} \mathcal{C} \rightarrow \mathcal{C}$ is the projection onto the $j$ 'th factor, then

$$
\omega_{\mathcal{C} / D}^{\otimes 2}=p_{1}^{*} \omega_{\mathcal{C} / D} \otimes p_{2}^{*} \omega_{\mathcal{C} / D}
$$

The formal correlation function $\langle\widetilde{\Phi}(q)|\widetilde{T}(z)| u\rangle d z^{2}$ which will be later proved to be an element of $H^{0}\left(\left(\mathcal{C}, \omega_{\mathcal{C} / D}\right)^{\otimes 2}\left(* \sum_{j=1}^{N} s_{j}(D)\right)\right.$ is defined in (4.19):

$$
\langle\widetilde{\Phi}(q)|\widetilde{T}(z)| u\rangle d z^{2}=\lim _{w \rightarrow z} \frac{1}{2}\left\{\left\langle\widetilde{\Phi}(q)\left|\frac{d \psi(w)}{d w} \bar{\psi}(z)\right| u\right\rangle d w d z-\omega(w, z)\langle\widetilde{\Phi}(q) \mid u\rangle d w d z\right\} .
$$

Then, by (4.21) we have that

$$
\langle\widetilde{\Phi}(q)|\widetilde{T}(z)| u\rangle d z^{2}=\langle\widetilde{\Phi}(q)|T(z)| u\rangle d z^{2}+\frac{1}{6}\langle\widetilde{\Phi}(q) \mid u\rangle S_{\omega}(z) d z^{2} .
$$

Let $\tilde{l}=l(z) \frac{d}{d z}$ be the meromorphic vector field defined in Lemma 5.6. For $q \neq 0$

$$
\tilde{l} \cdot\langle\widetilde{\Phi}(q)|\widetilde{T}(z)| u\rangle d z^{2}=l(z)\langle\widetilde{\Phi}(q)|\widetilde{T}(z)| u\rangle d z
$$

is a meromorphic one-form on

$$
C_{q}^{\prime}=C_{q} \backslash\{(x, y, q) \in S|| x|\leq \epsilon,| y \mid \leq \epsilon\}
$$

where $\epsilon<1$ is a sufficiently small positive number.

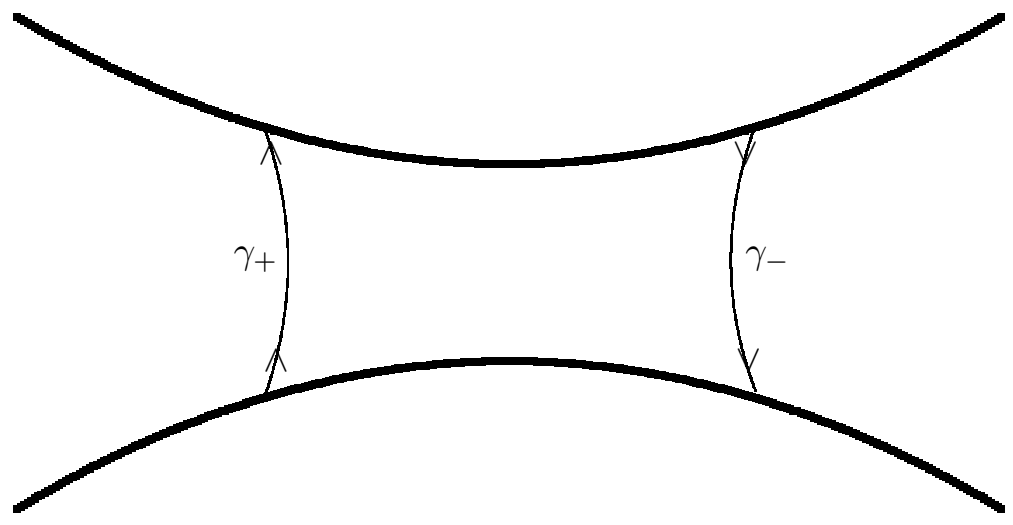


The boundary of $C_{q}^{\prime}$ consists of two disjoint curves $\gamma_{ \pm}$. We choose the orientation of $\gamma_{ \pm}$in such a way that $C_{q}^{\prime}$ lies to the left of $\gamma_{ \pm}$. Then, we have that

$$
\begin{aligned}
& \frac{1}{2 \pi \sqrt{-1}} \int_{\gamma_{+}} l(z)\langle\widetilde{\Phi}(q)|\widetilde{T}(z)| u\rangle d z+\frac{1}{2 \pi \sqrt{-1}} \int_{\gamma_{-}} l(w)\langle\widetilde{\Phi}(q)|\widetilde{T}(w)| u\rangle d w \\
& =\sum_{j=1}^{N} \operatorname{Res}_{Q_{j}}\{l(z)\langle\widetilde{\Phi}(q)|\widetilde{T}(z)| u\rangle d z\} \\
& \quad=\sum_{j=1}^{N}\left\langle\widetilde{\Phi}(q) \mid \operatorname{Res}_{\xi_{j}=0}\left(l\left(\xi_{j}\right) T\left(\xi_{j}\right) d \xi_{j}\right) u\right\rangle+\frac{1}{6} \sum_{j=1}^{N}{\underset{\xi}{\xi_{j}=0}}_{\operatorname{Res}}\left\{l\left(\xi_{j}\right) S_{\omega}\left(\xi_{j}\right) d \xi_{j}\langle\widetilde{\Phi}(q) \mid u\rangle\right\} \\
& =\sum_{j=1}^{N}\left\langle\widetilde{\Phi}(q) \mid \rho_{j}\left(T\left[l\left(\xi_{j}\right)\right]\right) u\right\rangle+b_{\omega}(\tilde{l})\langle\widetilde{\Phi}(q) \mid u\rangle .
\end{aligned}
$$

On the curve $\gamma_{+}$

$$
\tilde{l}=\frac{1}{2} z \frac{d}{d z}
$$

Hence

$$
\begin{aligned}
& \frac{1}{2 \pi \sqrt{-1}} \int_{\gamma_{+}} l(z)\langle\widetilde{\Phi}(q)|\widetilde{T}(z)| u\rangle d z \\
& \quad=\frac{1}{4 \pi \sqrt{-1}} \int_{\gamma_{+}} z\left\{\langle\widetilde{\Phi}(q)|T(z)| u\rangle+\frac{1}{6} S_{\omega}(z)\langle\widetilde{\Phi}(q) \mid u\rangle\right\} d z .
\end{aligned}
$$

Since $S_{\omega}(z) d z^{2}$ is holomorphic at $z=0$

$$
\frac{1}{2 \pi \sqrt{-1}} \int_{\gamma_{+}} l(z)\langle\widetilde{\Phi}(q)|\widetilde{T}(z)| u\rangle d z=\frac{1}{4 \pi \sqrt{-1}} \int_{\gamma_{+}} z\langle\widetilde{\Phi}(q)|T(z)| u\rangle d z
$$

On the other hand

$$
\begin{aligned}
& \frac{1}{4 \pi \sqrt{-1}} \int_{\gamma_{+}} z\langle\widetilde{\Phi}(q)|T(z)| u\rangle d z \\
& \quad=\frac{1}{2} \sum_{p \in \mathbf{Z}} \sum_{d=0}^{\infty} \sum_{i=1}^{m_{d}} \frac{(-1)^{p+d}}{2 \pi \sqrt{-1}} \int_{\gamma_{+}} z\left\langle\Phi|T(z)| v_{i}(d, p) \otimes v^{i}(d,-p-1) \otimes u\right\rangle q^{d+p(p+1) / 2} d z \\
& \quad=\frac{1}{2} \sum_{p \in \mathbf{Z}} \sum_{d=0}^{\infty} \sum_{i=1}^{m_{d}}(-1)^{p+d}\left\langle\Phi \mid L_{0}\left(v_{i}(d, p)\right) \otimes v^{i}(d,-p-1) \otimes u\right\rangle q^{d+p(p+1) / 2} \\
& =\frac{1}{2} \sum_{p \in \mathbf{Z}} \sum_{d=0}^{\infty} \sum_{i=1}^{m_{d}}(-1)^{p+d}\left(d+\frac{p(p+1)}{2}\right)\left\langle\Phi \mid v_{i}(d, p) \otimes v^{i}(d,-p-1) \otimes u\right\rangle q^{d+p(p+1) / 2}
\end{aligned}
$$

Similarly

$$
\begin{aligned}
& \frac{1}{2 \pi \sqrt{-1}} \int_{\gamma_{-}} l(w)\langle\widetilde{\Phi}(q)|\widetilde{T}(w)| u\rangle d w \\
& \quad=\frac{1}{2} \sum_{p \in \mathbf{Z}} \sum_{d=0}^{\infty} \sum_{i=1}^{m_{d}}(-1)^{p+d}\left(d+\frac{p(p+1)}{2}\right)\left\langle\Phi \mid v_{i}(d, p) \otimes v^{i}(d,-p-1) \otimes u\right\rangle q^{d+p(p+1) / 2} .
\end{aligned}
$$


Thus we obtain that

$$
\begin{aligned}
& \sum_{j=1}^{N}\left\langle\widetilde{\Phi}(q) \mid \rho_{j}\left(T\left[l\left(\xi_{j}\right)\right]\right) u\right\rangle+b_{\omega}(\tilde{l})\langle\Phi \mid u\rangle \\
& \quad=\sum_{p \in \mathbf{Z}} \sum_{d=0}^{\infty} \sum_{i=1}^{m_{d}}(-1)^{p+d}\left(d+\frac{p(p+1)}{2}\right)\left\langle\Phi \mid v_{i}(d, p) \otimes v^{i}(d,-p-1) \otimes u\right\rangle q^{d+p(p+1) / 2} .
\end{aligned}
$$

On the other hand

$q \frac{d}{d q}\langle\widetilde{\Phi}(q) \mid u\rangle=\sum_{p \in \mathbf{Z}} \sum_{d=0}^{\infty} \sum_{i=1}^{m_{d}}(-1)^{p+d}\left(d+\frac{p(p+1)}{2}\right)\left\langle\Phi \mid v_{i}(d, p) \otimes v^{i}(d,-p-1) \otimes u\right\rangle q^{d+p(p+1) / 2}$.

Hence $\langle\widetilde{\Phi}(q) \mid u\rangle$ is a formal solution of the differential equation

$$
q \frac{d}{d q}\langle\widetilde{\Phi}(q) \mid u\rangle-\sum_{j=1}^{N}\left\langle\widetilde{\Phi}(q) \mid \rho_{j}\left(T\left[l\left(\xi_{j}\right)\right]\right) u\right\rangle-\frac{1}{6} b_{\omega}(\tilde{l})\langle\Phi \mid u\rangle=0
$$

which is of Fuchsian type. Hence $\langle\widetilde{\Phi}(q) \mid u\rangle$ converges for all $u \in \mathcal{F}_{N}$.

Now let us give a proof of Theorem 3.2.

By (4.2) and Theorem $4.1 \operatorname{dim} \mathcal{V}^{\dagger}$ ab $(\mathfrak{X})$ depends only on the genus of the curve $C$ and the number $N$ of the points, if the curve $C$ is non-singular. Moreover, by Theorem 3.4 $\operatorname{dim} \mathcal{V}^{\dagger}{ }_{a b}(\mathfrak{X})$ depends only on the genus of the curve $C$ provided $C$ is non-singular. Put

$$
d(g)=\operatorname{dim} \mathcal{V}_{\mathrm{ab}}^{\dagger}(\mathfrak{X})
$$

for a non-singular curve $C$ of genus $g$.

Now assume that the curve $C$ has one double point $P$. Let $\mathfrak{F}=\left(\pi: \mathcal{C} \rightarrow D ; s_{+}, s_{-}, \xi_{1}, \ldots, \xi_{N}\right)$ be the family constructed above so that $\mathfrak{F}(q), q \neq 0$ is a non-singular curve of genus $g$. Then, by (4.2) and Proposition 4.1

$$
d(g)=\operatorname{dim} \mathcal{V}_{\mathrm{ab}}^{\dagger}(\mathfrak{X})=\operatorname{dim} \mathcal{V}_{\mathrm{ab}}(\mathfrak{F}(0)) \geq \operatorname{dim} \mathcal{V}_{\mathrm{ab}}(\mathfrak{F}(q))=\operatorname{dim} \mathcal{V}_{\mathrm{ab}}^{\dagger}(\mathfrak{F}(q)) .
$$

Moreover, by Theorem 3.5 we have that

$$
\operatorname{dim} \mathcal{V}_{\mathrm{ab}}^{\dagger}(\mathfrak{X})=\operatorname{dim} \mathcal{V}_{\mathrm{ab}}^{\dagger}(\widetilde{\mathfrak{X}})=d(g-1) .
$$

On the other hand by Theorem 5.1

$$
\operatorname{dim} \mathcal{V}_{\mathrm{ab}}^{\dagger}(\mathfrak{F}(0)) \leq \operatorname{dim} \mathcal{V}_{\mathrm{ab}}^{\dagger}(\mathfrak{F}(q)) .
$$

Hence

$$
d(g-1)=\operatorname{dim} \mathcal{V}^{\dagger}{ }_{\mathrm{ab}}(\mathfrak{F}(0)) \leq \operatorname{dim} \mathcal{V}^{\dagger}{ }_{\mathrm{ab}}(\mathfrak{F}(q))=d(g) .
$$

If the curve $C$ has $m$ double points, applying Theorem $3.5 \mathrm{~m}$ times we conclude that $\operatorname{dim} \mathcal{V}_{a b}^{\dagger}(\mathfrak{X})$ is independent of $\mathfrak{X}$. In Example 3.1, we showed that $\operatorname{dim} \mathcal{V}^{\dagger}{ }_{\mathrm{ab}}\left(\left(\mathbf{P}^{1} ; 0 ; z\right)\right)=1$. Hence, by Theorem 3.4 for any $\mathfrak{X}$ we have that $\operatorname{dim} \mathcal{V}^{\dagger}{ }_{\mathrm{ab}}(\mathfrak{X})=1$.

QED 
Corollary 5.1. The connection (5.12) extends holomorphically over $q=0$.

Proof. Since $\langle\widetilde{\Phi}(q)|$ is a single valued holomorphic function and the differential equation (5.12) is of Fuchsian type,

$$
\sum_{j=1}^{N}\left\langle\widetilde{\Phi}(q) \mid \rho_{j}\left(T\left[\underline{l}_{j}\right]\right) u\right\rangle+\frac{1}{6} b_{\omega}(\vec{l})\langle\widetilde{\Phi}(q) \mid u\rangle
$$

must be divisible by $q$. Hence the differential equation has no singularity at $q=0$. QED

Theorem 3.2 implies the following important fact.

Theorem 5.2. For a family

$$
\mathfrak{F}=\left(\pi: \mathcal{C} \rightarrow \mathcal{B} ; s_{1}, \ldots, s_{N} ; \xi_{1}, \ldots, \xi_{N}\right)
$$

of $N$-pointed semi-stable curves with formal neighbourhoods the sheaf $\mathcal{V}^{\dagger}{ }_{a b}(\mathfrak{F})$ of ghost vacua and the sheaf $\mathcal{V}_{\mathrm{ab}}(\mathfrak{F})$ of dual ghost vacua are invertible $\mathcal{O}_{\mathcal{B}}$-modules. Moreover, they are dual to each other.

Proof. By Theorem 3.2 and (4.2), for any point $b \in \mathcal{B}$ the vector space $\mathcal{V}_{\mathrm{ab}}(\mathfrak{F}) \otimes_{\mathcal{O}_{\mathcal{B}}}$ $\mathcal{O}_{\mathcal{B}, b} / \mathfrak{m}_{b}$ is one-dimensional. As $\mathcal{V}_{a b}(\mathfrak{F})$ is a coherent $\mathcal{O}_{\mathcal{B}}$-module, this implies that $\mathcal{V}_{a b}(\mathfrak{F})$ is an invertible $\mathcal{O}_{\mathcal{B}}$-module. Then, by (4.1) $\mathcal{V}^{\dagger}{ }_{a b}(\mathfrak{F})$ is also an invertible $\mathcal{O}_{\mathcal{B}}$-module. QED

Next we shall consider smoothing of a family of nodal curves with sections and formal coordinates. We need to generalize the above construction.

Let

$$
\widetilde{\mathfrak{F}}=\left(\widetilde{\pi}: \widetilde{\mathcal{C}} \rightarrow E ; s_{1}, \ldots, s_{N}, t_{+}, t_{-} ; \xi_{1}, \ldots, \xi_{N}, z, w\right)
$$

be a family of $(N+2)$-pointed smooth curves with formal coordinates. Assume that $N \geq 1$ and $E$ is a small polydisk $\left\{\left(u_{1}, \ldots, u_{m}\right) \in \mathbf{C}^{m}|| u_{i} \mid<\varepsilon, 1 \leq i \leq m\right\}$, and $z$, $w$ are holomorphic coordinates such that there exists a meromorphic vector field $\widetilde{l} \in$ $H^{0}\left(\widetilde{C}, \Theta_{\widetilde{\mathcal{C}} / E}(* S)\right)$ with

$$
\widetilde{l}_{X}=\frac{1}{2} z \frac{d}{d z}, \quad \widetilde{l}_{Y}=\frac{1}{2} w \frac{d}{d w} .
$$

There always exist such coordinates by Lemma [5.6. Put

$$
S=\sum_{j=1}^{N} s_{j}(E), \quad S_{ \pm}=t_{+}(E)+t_{-}(E) .
$$

Then for any positive integer $M$ we have and exact sequence

$$
0 \rightarrow \Theta_{\widetilde{\mathcal{C}} / E}\left(-S_{ \pm}+M S\right) \rightarrow \Theta_{\widetilde{\mathcal{C}}}\left(-S_{ \pm}+M S\right) \stackrel{d \widetilde{\pi}}{\rightarrow} \widetilde{\pi}^{*} \Theta_{E} \rightarrow 0
$$

where $\Theta_{\widetilde{\mathcal{C}} / E}$ is the sheaf of relative holomorphic vector fields and $\Theta_{Z}$ denotes the sheaf of holomorphic vector fields on some complex manifold $Z$. Put

$$
\Theta_{\widetilde{\mathcal{C}}}\left(-S_{ \pm}+M S\right)_{\tilde{\pi}}=d \widetilde{\pi}^{-1}\left(\widetilde{\pi}^{-1} \Theta_{E}\right) .
$$

Taking the inductive limit on $M$ we can also define $\Theta_{\widetilde{\mathcal{C}}}\left(-S_{ \pm}+* S\right)_{\tilde{\pi}}$. 
Similarly for any positive integer $M$ we have an exact sequence

$$
0 \rightarrow \Theta_{\widetilde{\mathcal{C}} / E}\left(-S_{ \pm}-S\right) \rightarrow \Theta_{\widetilde{\mathcal{C}} / E}\left(-S_{ \pm}+M S\right) \rightarrow \bigoplus_{j=1}^{N} \bigoplus_{k=0}^{M} \mathcal{O}_{E} \xi_{j}^{-k} \frac{d}{d \xi_{j}} \rightarrow 0
$$

From these exact sequences we have a commutative diagram

$$
\begin{aligned}
& 0 \rightarrow \tilde{\pi}_{*} \Theta_{\widetilde{\mathcal{C}} / E}\left(-S_{ \pm}+* S\right) \quad \rightarrow \quad \widetilde{\pi}_{*} \Theta_{\widetilde{\mathcal{C}}}\left(-S_{ \pm}+* S\right)_{\widetilde{\pi}} \stackrel{d \widetilde{\pi}}{\rightarrow} \quad \Theta_{E} \quad \rightarrow 0 \\
& \| \quad \downarrow_{p} \quad \downarrow_{\rho} \\
& 0 \rightarrow \widetilde{\pi}_{*} \Theta_{\widetilde{\mathcal{C}} / E}\left(-S_{ \pm}+* S\right) \rightarrow \bigoplus_{j=1}^{N} \mathcal{O}_{E}\left[\xi_{j}^{-1}\right] \frac{d}{d \xi_{j}} \quad \rightarrow \quad R^{1} \widetilde{\pi}_{*} \Theta_{\widetilde{C} / E}\left(-S_{ \pm}-S\right) \rightarrow 0
\end{aligned}
$$

For simplicity assume that the $\mathcal{O}_{E}$-homomorphism $\rho$ is injective. Therefore, $p$ is also injective. Let

$$
\widetilde{p}: \widetilde{\pi}_{*} \Theta_{\widetilde{\mathcal{C}}}\left(-S_{ \pm}+* S\right)_{\widetilde{\pi}} \rightarrow \bigoplus_{j=1}^{N} \mathcal{O}_{E}\left(\left(\xi_{j}\right)\right) \frac{d}{d \xi_{j}}
$$

be the Laurent expansions of the $\frac{d}{d \xi_{j}}$ part along $s_{j}(E)$ and put

$$
\mathcal{L}(\widehat{\mathfrak{F}})=\widetilde{p}\left(\widetilde{\pi}_{*} \Theta_{\widetilde{\mathcal{C}}}\left(-S_{ \pm}+* S\right)_{\tilde{\pi}}\right) \subset \bigoplus_{j=1}^{N} \mathcal{O}_{E}\left(\left(\xi_{j}\right)\right) \frac{d}{d \xi_{j}} .
$$

Then we have an exact sequence

$$
0 \rightarrow \widetilde{\pi}_{*} \Theta_{\widetilde{\mathcal{C}} / E}\left(-S_{ \pm}+* S\right) \rightarrow \mathcal{L}(\widehat{\mathfrak{F}}) \stackrel{\widehat{\theta}}{\rightarrow} \Theta_{E} \rightarrow 0 .
$$

Hence we can find local vector fields

$$
\vec{m}^{(i)}=\left(m_{1}^{(i)} \frac{d}{d \xi_{1}}, \ldots, m_{N}^{(i)} \frac{d}{d \xi_{N}}\right)
$$

which are sections of $\mathcal{L}(\widehat{\mathfrak{F}})$ such that

$$
\widehat{\theta}\left(\vec{m}^{(i)}\right)=\frac{d}{d u_{i}}
$$

Note that by (4.9) we can define

$$
\mathcal{L}(\widetilde{\mathfrak{F}}) \subset\left(\bigoplus_{j=1}^{N} \mathcal{O}_{E}\left(\left(\xi_{j}\right)\right) \frac{d}{d \xi_{j}}\right) \oplus \mathcal{O}_{E}((z)) \frac{d}{d z} \oplus \mathcal{O}_{E}((w)) \frac{d}{d w}
$$

and we have the exact sequence

$$
0 \rightarrow \widetilde{\pi}_{*} \Theta_{\widetilde{\mathcal{C}} / E}(* S) \rightarrow \mathcal{L}(\widehat{\mathfrak{F}}) \stackrel{\widetilde{\theta}}{\rightarrow} \Theta_{E} \rightarrow 0
$$


Then the above argument shows that if we put

$$
\overrightarrow{\vec{m}}^{(i)}=\left(m_{1}^{(i)} \frac{d}{d \xi_{1}}, \ldots, m_{N}^{(i)} \frac{d}{d \xi_{N}}, 0,0\right),
$$

then

$$
\widetilde{\theta}\left(\overrightarrow{\vec{m}}^{(i)}\right)=\frac{d}{d u_{i}} .
$$

Let us identify $t_{+}(u)$ with $t_{-}(u)$ for each $u \in E$ and obtain a family $\widehat{\mathfrak{F}}=(\widehat{\pi}: \widehat{\mathcal{C}} \rightarrow$ $\left.E ; s_{1}, \ldots, s_{N} ; \xi_{1}, \ldots, \xi_{N}\right)$ of $N$-pointed nodal curves. Let $\nu: \widetilde{\mathcal{C}} \widehat{\mathcal{C}}$ be this identification map. A flat deformation of this family $\widehat{\mathfrak{F}}$ can be constructed as follows. For further details see section 5.3 in [U2].

For $0<\varepsilon \leq 1$ put

$$
\begin{aligned}
& X_{\varepsilon}=\{P \in \widetilde{\mathcal{C}}|| z(P) \mid<\varepsilon\} \\
& Y_{\varepsilon}=\{P \in \widetilde{\mathcal{C}}|| w(P) \mid<\varepsilon\}
\end{aligned}
$$

and also put $X=X_{1}, Y=Y_{1}$. Choose $0<\varepsilon_{1}<\varepsilon_{2}<1$ and choose an open covering $\left\{U_{\alpha}\right\}_{4 \leq \alpha \leq s}$ of $\widetilde{\mathcal{C}} \backslash\left(X_{\varepsilon_{2}} \cup Y_{\varepsilon_{2}}\right)$ such that

$$
U_{\alpha} \cap X_{\varepsilon_{1}}=\emptyset, \quad U_{\alpha} \cap Y_{\varepsilon_{1}}=\emptyset .
$$

Put

$$
\begin{aligned}
D & =\{q \in \mathbf{C}|| q \mid<1\} \\
S_{0} & =\left\{(x, y, q) \in \mathbf{C}^{3}|x y=q,| x|<1,| y|<1,| q \mid<1\right\} \\
\mathcal{S} & =S_{0} \times E \\
Z & =\{(P, q) \in \widetilde{\mathcal{C}} \times D \mid P \in \widetilde{\mathcal{C}} \backslash(X \cup Y), \text { or } P \in X \&|z(P)|>|q|\} \\
W & =\{(P, q) \in \widetilde{\mathcal{C}} \times D \mid P \in \widetilde{\mathcal{C}} \backslash(X \cup Y), \text { or } P \in Y \&|w(P)|>|q|\} .
\end{aligned}
$$

On $Z \cup \mathcal{S} \cup W$ let us introduce an equivalence relation $\sim$ as follows:.

1. A point $(P, q) \in Z \cap(X \times D)$ and a point $\left(x, y, q^{\prime}, u\right) \in \mathcal{S}$ are equivalent if and only if

$$
\left(x, y, q^{\prime}, u\right)=\left(z(P), \frac{q}{z(P)}, q, \tilde{\pi}(P)\right) .
$$

2. A point $(P, q) \in W \cap(Y \times D)$ and a point $\left(x, y, q^{\prime}, u\right) \in \mathcal{S}$ are equivalent if and only if

$$
\left(x, y, q^{\prime}, u\right)=\left(\frac{q}{w(P)}, w(P), q, \widetilde{\pi}(P)\right) .
$$

3. A point $(P, q) \in Z$ and a point $\left(Q, q^{\prime}\right) \in W$ are equivalent if and only if

$$
(P, q)=\left(Q, q^{\prime}\right) \text {. }
$$

Now put $\mathcal{C}=Z \cup \mathcal{S} \cup W / \sim$. Then, $\mathcal{C}$ is a complex manifold and there is a natural holomorphic mapping $\pi: \mathcal{C} \rightarrow E \times D$. Moreover $(\widetilde{\mathcal{C}} \backslash(X \cup Y)) \times D$ is contained in $\mathcal{C}$ as an open subset. Hence we can define holomorphic sections $s_{j}$ by

$$
\begin{aligned}
s_{j}: E \times D & \rightarrow \mathcal{C} \\
(u, q) & \rightarrow\left(s_{j}(u), q\right) \in Z .
\end{aligned}
$$


Then it is easy to show that $\mathfrak{F}=\left(\pi: \mathcal{C} \rightarrow \mathcal{B}=E \times D ; s_{1}, \ldots, s_{N} ; \xi_{1}, \ldots, \xi_{N}\right)$ is a family of $N$-pointed curves with formal coordinates. It is also easy to show that the family $\pi: \mathcal{C} \rightarrow \mathcal{B}=E \times D$ is a versal family of $N$-pointed curves. Note that each fiber over $q=0$ is a nodal curve. We identify $E$ with $E \times\{0\}$.

Then we have an exact sequence.

$$
0 \rightarrow \pi_{*} \Theta_{\mathcal{C} / \mathcal{B}}(* S) \rightarrow \mathcal{L}(\mathfrak{F}) \stackrel{\theta}{\rightarrow} \Theta_{\mathcal{B}}(-\log E) \rightarrow 0 .
$$

Lemma 5.8. We can choose a local vector field $\vec{m}^{(i)}$ in (5.14) in such a way that it can be regarded as a section of $\mathcal{L}(\mathfrak{F})$ over $\mathcal{B}$ with

$$
\theta\left(\vec{m}^{(i)}\right)=\frac{d}{d u_{i}}
$$

Proof. Since $\frac{d}{d u_{i}} \in H^{0}\left(\mathcal{B}, \Theta_{\mathcal{B}}(-\log E)\right)$, we can choose a $\tau^{(i)} \in \pi_{*} \Theta_{\mathcal{C}, \pi}^{\prime}(* S)$ such that $\theta\left(\widetilde{p}\left(\tau^{(i)}\right)\right)=\frac{d}{d u_{i}}$, provided we choose $\mathcal{B}$ small enough.

Let $\left\{U_{\alpha}\right\}_{4 \leq \alpha \leq s}$ be the open covering of $\widetilde{C} \backslash\left\{X_{\varepsilon} \cup Y_{\varepsilon_{2}}\right\}$ chosen above. Put

$$
\begin{aligned}
& \mathcal{U}_{0}=\left\{\left(x, y, u_{1}, \ldots, u_{n}, q\right) \in \mathcal{S} \mid x \neq 0\right\}, \quad \mathcal{U}_{1}=\left\{\left(x, y, u_{1}, \ldots, u_{n}, q\right) \in \mathcal{S} \mid y \neq 0\right\} \\
& \mathcal{U}_{2}=X \times D, \quad \mathcal{U}_{3}=Y \times D, \quad \mathcal{U}_{\alpha}=U_{\alpha} \times D, \quad 4 \leq \alpha \leq s .
\end{aligned}
$$

Then, $\left\{\mathcal{U}_{\alpha}\right\}_{1 \leq \alpha \leq s}$ is an open covering on $\mathcal{C}$. Let $\left(w_{\alpha}, u_{1}, \ldots, u_{m}, q\right)$ be local coordinates of $\mathcal{U}_{\alpha}$, where

$$
w_{0}=y, \quad w_{1}=x, \quad w_{2}=x, \quad w_{3}=y .
$$

In these coordinates our vector field $\tau^{(i)}$ is expressed as

$$
\left.\tau^{(i)}\right|_{\mathcal{U}_{\alpha}}=A_{\alpha}\left(w_{\alpha}, q, u\right) \frac{d}{d w_{\alpha}}+\frac{d}{d u_{i}}+B(u, q) q \frac{d}{d q} .
$$

On the other hand the Kodaira-Spencer class $\rho\left(\frac{d}{d u_{i}}\right)$ is expressed by a $\check{C}$ ech cocycle

$$
\theta_{\alpha \beta}=\frac{\partial f_{\alpha \beta}}{\partial u_{i}} \frac{d}{d w_{\alpha}}
$$

where on $\mathcal{U}_{\alpha} \cap \mathcal{U}_{\beta} \neq \emptyset$ we write

$$
w_{\alpha}=f_{\alpha \beta}\left(w_{\beta}, u_{1}, \ldots, u_{m}, q\right) .
$$

Hence, if we put

$$
\theta_{\alpha}=-A_{\alpha}\left(w_{\alpha}, q, u\right) \frac{d}{d w_{\alpha}}-B(u, q) q \frac{d}{d q}
$$

then we have

$$
\theta_{\alpha \beta}=\theta_{\beta}-\theta_{\alpha}
$$

Note that for $\alpha, \beta \leq 4, f_{\alpha \beta}$ is independent of $q$. Therefore on $\left\{\widetilde{\mathcal{C}} \backslash\left\{X_{\varepsilon_{2}} \cup Y_{\varepsilon_{2}}\right\}\right\} \times D$

$$
A_{\alpha}\left(w_{\alpha}, 0, u\right) \frac{d}{d w_{\alpha}}+\frac{d}{d u_{i}}+B(u, 0) q \frac{d}{d q}, \quad \alpha \geq 4
$$


define a global meromorphic vector filed $\widetilde{\tau}^{(i)^{\prime}}$.

On the other hand for $\alpha, \beta \leq 3, f_{\alpha \beta}$ is independent of $u_{1}, \ldots, u_{m}$. On $\mathcal{U}_{0} \cap \mathcal{U}_{2}$ we have

$$
w_{2}=q / w_{0} .
$$

Hence we have

$A_{0}\left(w_{0}, u, q\right) \frac{d}{d w_{0}}+\frac{d}{d u_{i}}+B(u, q) q \frac{d}{d q}=\left(-\frac{w_{2}}{w_{0}} A_{0}\left(q / w_{2}, u, q\right)+w_{2} B(u, q)\right) \frac{d}{d w_{2}}+\frac{d}{d u_{i}}+B(u, q) q \frac{d}{d q}$.

Therefore

$$
A_{2}\left(w_{2}, u, q\right)=-\frac{w_{2}}{w_{0}} A_{0}\left(q / w_{2}, u, q\right)+w_{2} B(u, q) .
$$

Since $A_{0}\left(w_{0}, u, q\right)$ and $A_{2}\left(w_{2}, u, q\right)$ are holomorphic, we conclude that $A_{0}\left(w_{1}, u, q\right)$ has the form

$$
A_{0}\left(w_{0}, u, q\right)=a_{0}(u, q) w_{0}
$$

and on $\mathcal{U}_{0} \cap \mathcal{U}_{2}$

$$
A_{2}\left(w_{2}, u, q\right)=\left(-a_{0}(u, q)+B(u, q)\right) w_{2},
$$

where $a_{0}(u, q)$ is holomorphic in $u$ and $q$. In this way we can show that on $\mathcal{U}_{\alpha}, \alpha \leq 3, A_{\alpha}$ has the form

$$
A_{\alpha}\left(w_{\alpha}, u, q\right)=a_{\alpha}(u, q) w_{\alpha}
$$

where $a_{\alpha}(u, q)$ is holomorphic in $u$ and $q$. Also the above calculations show that

$$
a_{\alpha}(u, 0) w_{\alpha} \frac{d}{d w_{\alpha}}+\frac{d}{d u_{i}}+B(u, 0) q \frac{d}{d q}, \quad \alpha \leq 3
$$

define a holomorphic vector field on $\cup_{\alpha=0}^{3} \mathcal{U}_{\alpha}$. This vector field coincides with $\widetilde{\tau}^{(i)^{\prime}}$ on the intersection of $\left\{\widetilde{\mathcal{C}} \backslash\left\{X_{\varepsilon} \cup Y_{\varepsilon_{2}}\right\}\right\} \times D$ and $\cup_{\alpha=0}^{3} \mathcal{U}_{\alpha}$. Thus we have a global meromorphic vector field $\widetilde{\tau}^{(i)}$ on $\mathcal{C}$. Then, by our construction if we put $\vec{m}^{(i)}=\widetilde{p}\left(\widetilde{\tau}^{(i)}\right)$, it is independent of $q$ and we have $\theta\left(\vec{m}^{(i)}\right)=\frac{d}{d u_{i}}$.

Now restrict $\widetilde{\tau}^{(i)}$ to $q=0$ and denote this restriction $\widetilde{\tau}^{(i)}(0)$. Then the above calculation shows that $\widetilde{\tau}^{(i)}(0) I \subset I$ where $I$ is the ideal defining the double point. Hence, $\nu^{*}\left(\widetilde{\tau}^{(i)}(0)\right)$ is a meromorphic vector field on $\widetilde{C}$ and $\tilde{\theta}\left(\tilde{p}\left(\nu^{*} \widetilde{\tau}^{(i)}(0)\right)\right)=\frac{d}{d u_{i}}$. Note that $\tilde{p}\left(\nu^{*} \widetilde{\tau}^{(i)}(0)\right)=\vec{m}^{(i)}$.

QED

Now from $\langle\Phi| \in \mathcal{V}^{\dagger}{ }_{\mathrm{ab}}(\widetilde{\mathfrak{F}})$ we can construct $\langle\Psi(q)| \in \mathcal{V}^{\dagger}$ ab $(\mathfrak{F})$ using formula (5.10):

$$
\langle\widetilde{\Phi}(q) \mid u\rangle=\sum_{p \in \mathbf{Z}}\left\{\sum_{d=0}^{\infty} \sum_{i=1}^{m_{d}}(-1)^{p+d}\left\langle\Phi \mid v_{i}(d, p) \otimes v^{i}(d,-p-1) \otimes u\right\rangle\right\} q^{d+p(p+1) / 2} .
$$

Then by (4.12), (5.14) and (5.15)

$$
\begin{aligned}
& \left\langle\widetilde{\Phi}(q)\left|D\left(\vec{m}^{(i)}\right)\right| u\right\rangle=-\sum_{j=1}^{N}\left\langle\widetilde{\Phi}(q)\left|\rho_{j}\left(T\left[m_{j}^{(i)}\right]\right)\right| u\right\rangle \\
& =-\sum_{j=1}^{N} \sum_{p \in \mathbf{Z}}\left\{\sum_{d=0}^{\infty} \sum_{i^{\prime}=1}^{m_{d}}(-1)^{p+d}\left\langle\Phi \mid \rho_{j}\left(T\left[m_{j}^{(i)}\right]\right) v_{i^{\prime}}(d, p) \otimes v^{i^{\prime}}(d,-p-1) \otimes u\right\rangle\right\} q^{d+p(p+1) / 2} \\
& =-\sum_{p \in \mathbf{Z}}\left\{\sum_{d=0}^{\infty} \sum_{i^{\prime}=1}^{m_{d}}(-1)^{p+d}\left\langle\Phi \mid D\left(\overrightarrow{\vec{m}}^{(i)}\right) v_{i^{\prime}}(d, p) \otimes v^{i^{\prime}}(d,-p-1) \otimes u\right\rangle\right\} q^{d+p(p+1) / 2} .
\end{aligned}
$$


Let $\omega(q)=\omega(x, y, u . q) d x d y \in H^{0}\left(\mathcal{C} \otimes_{\widetilde{E} \times D} \mathcal{C}, \omega^{\bowtie 2}(2 \Delta)\right)$ be a bidifferential with $\operatorname{Res}^{2}(\omega)=1$. Note that $\omega(0)$ is a bidifferential on $\widetilde{\mathcal{C}} \times_{E} \widetilde{\mathcal{C}}$.

Then, by (4.24) the connection $\nabla_{\frac{d}{d u_{i}}}^{(\omega(q))}$ is defined by

$$
\nabla_{\frac{d}{d u_{i}}}^{(\omega(q))}\langle\widetilde{\Phi}(q)|=D\left(\vec{m}^{(i)}\right)\langle\widetilde{\Phi}(q)|+\frac{1}{6} b_{\omega(q)}\left(\vec{m}^{(i)}\right)\langle\widetilde{\Phi}(q)| .
$$

Therefore, if we start from $\langle\Psi|=\nabla_{\frac{d}{d u_{i}}}^{(\omega(0))}\langle\Phi| \in \mathcal{V}^{\dagger}{ }_{\mathrm{ab}}(\widetilde{\mathfrak{F}})$ and construct $\langle\widetilde{\Psi}(q)| \in \mathcal{V}^{\dagger}{ }_{\mathrm{ab}}(\mathfrak{F})$ by the sewing procedure, we have that

$$
\langle\widetilde{\Psi}(q)|=D\left(\vec{m}^{(i)}\right)\langle\widetilde{\Phi}(q)|+\frac{1}{6} b_{\omega(0)}\left(\vec{m}^{(i)}\right)\langle\widetilde{\Phi}(q)| .
$$

Therefore, we have

$$
\nabla_{\frac{d}{d u_{i}}}^{(\omega(q))}\langle\widetilde{\Phi}(q)|-\langle\widetilde{\Psi}(q)|=\frac{1}{6}\left(b_{\omega(q)}\left(\vec{m}^{(i)}\right)-b_{\omega(0)}\left(\vec{m}^{(i)}\right)\right)\langle\widetilde{\Phi}(q)| .
$$

Thus we obtain the following theorem.

Theorem 5.3. Let $\mathfrak{F}=\left(\pi: \mathcal{C} \rightarrow \mathcal{B}=E \times D ; s_{1}, \ldots, s_{N} ; \xi_{1}, \ldots, \xi_{N}\right)$ be a family of $N$ pointed curves with formal coordinates such that the restriction of the family to $E$ is the family of nodal curves $\mathfrak{F}=\left(\pi: \widehat{\mathcal{C}} \rightarrow E ; s_{1}, \ldots, s_{N} ; \xi_{1}, \ldots, \xi_{N}\right)$. Let

$$
\widetilde{\mathfrak{F}}=\left(\widetilde{\pi}: \widetilde{\mathcal{C}} \rightarrow E ; s_{1}, \ldots, s_{N}, t_{+}, t_{-} ; \xi_{1}, \ldots, \xi_{N}, z, w\right)
$$

be the family of $(N+2)$-pointed curves obtained by normalization of each fiber of $\widehat{\mathfrak{F}}$. For $\langle\Phi| \in \mathcal{V}^{\dagger}{ }_{\mathrm{ab}}(\widetilde{\mathfrak{F}})$ let $\langle\widetilde{\Phi}(q)| \in \mathcal{V}^{\dagger}{ }_{\mathrm{ab}}(\mathfrak{F})$ be obtained by sewing. Let $\omega(q) \in H^{0}\left(\mathcal{C} \otimes_{E \times D}\right.$ $\left.\mathcal{C}, \omega^{\bowtie 2}(2 \Delta)\right)$ be a bidifferential with $\operatorname{Res}^{2}(\omega)=1$. Let $\langle\widetilde{\Psi}(q)| \in \mathcal{V}^{\dagger}$ ab $(\mathfrak{F})$ be constructed from $\langle\Psi|=\nabla_{\frac{d}{d u_{i}}}^{(\omega(0))}\langle\Phi| \in \mathcal{V}^{\dagger}{ }_{\mathrm{ab}}(\widetilde{\mathfrak{F}})$ by the sewing. Then we have that

$$
\nabla_{\frac{d}{d u_{i}}}^{(\omega(q))}\langle\widetilde{\Phi}(q)|-\langle\widetilde{\Psi}(q)|=\frac{1}{6}\left(b_{\omega(q)}\left(\vec{m}^{(i)}\right)-b_{\omega(0)}\left(\vec{m}^{(i)}\right)\right)\langle\widetilde{\Phi}(q)| .
$$

Remark 5.1. For the non-abelian case the same result holds, provided one replaces the coefficient $1 / 6$ by $c_{v} / 12$.

\section{Formal Coordinates and Preferred Sections}

In this section we shall study the behavior of the ghost vacua under coordinate changes. We shall also define a preferred section which is a local holomorphic section of the sheaf of ghost vacua $\mathcal{V}^{\dagger}{ }_{a b}(\mathfrak{F})$ of a family of one-pointed smooth curves with formal coordinates.

First we recall some basic facts about the relationship between coordinates change and local vector fields.

We let $\mathcal{D}$ be the automorphism group Aut $\mathbf{C}((\xi))$ of the field $\mathbf{C}((\xi))$ of formal Laurent series as a $\mathbf{C}$-algebra. The group $\mathcal{D}$ may be regarded as the automorphism group of the ring $\mathbf{C}[[\xi]]$ of formal power series. There is a natural isomorphism

$$
\begin{aligned}
\mathcal{D} & \simeq\left\{\sum_{n=0}^{\infty} a_{n} \xi^{n+1} \mid a_{0} \neq 0\right\} \\
h & \mapsto h(\xi)
\end{aligned}
$$


where the composition $h \circ g$ of $h, g \in \mathcal{D}$ corresponds to a formal power series $h(g(\xi))$. In the following we identify $\mathcal{D}$ with $\left\{\sum_{n=0}^{\infty} a_{n} \xi^{n+1} \mid a_{0} \neq 0\right\}$.

Put

$$
\mathcal{D}^{p}=\left\{h \in \mathcal{D} \mid h(\xi)=\xi+a_{p} \xi^{p+1}+\cdots\right\}
$$

for a positive integer $p$. Then we have a filtration

$$
\mathcal{D}=\mathcal{D}^{0} \supset \mathcal{D}^{1} \supset \mathcal{D}^{2} \supset \ldots
$$

Put also

$$
\begin{aligned}
\underline{d} & =\mathbf{C}[[\xi]] \xi \frac{d}{d \xi} \\
\underline{d}^{p} & =\mathbf{C}[[\xi]] \xi^{p+1} \frac{d}{d \xi} \quad p=0,1,2, \ldots
\end{aligned}
$$

Then, we have a filtration

$$
\underline{d}=\underline{d}^{0} \supset \underline{d}^{1} \supset \underline{d}^{2} \supset \cdots
$$

For any $\underline{l} \in \underline{d}$ and $f(\xi) \in \mathbf{C}[[\xi]]$ define $\exp (\underline{l})(f(\xi))$ by

$$
\exp (\underline{l})(f(\xi))=\sum_{k=0}^{\infty} \frac{1}{k !}\left(\underline{k}^{k} f(\xi)\right) .
$$

Also put

$$
\begin{array}{rlrl}
\mathcal{D}_{+}^{0} & =\left\{h \in \mathcal{D} \mid h(\xi)=a \xi+a_{1} \xi^{2}+\cdots,\right. & & a>0\} \\
\underline{d}_{+}^{0} & =\left\{l(\xi) \frac{d}{d \xi} \mid l(\xi)=\alpha \xi+\alpha_{1} \xi^{2}+\cdots,\right. & \alpha \in \mathbf{R}\}
\end{array}
$$

Then, we have the following result.

Lemma 6.1. The exponential map

$$
\begin{aligned}
\exp : \underline{d} & \rightarrow \mathcal{D} \\
\underline{l} & \mapsto \exp (\underline{l})
\end{aligned}
$$

is surjective. Moreover, the exponential map induces an isomorphism

$$
\exp : \underline{d}_{+}^{0} \simeq \mathcal{D}_{+}^{0} .
$$

Since, for any integer $n$, we have

$$
\exp \left(2 \pi n \sqrt{-1} \xi \frac{d}{d \xi}\right)=i d
$$

the exponential mapping is not injective on $\underline{d}$.

For the energy-momentum tensor $T(z)$ and any element $\underline{l} \in \mathcal{D}_{+}^{0}$ we define $\exp (T[\underline{l}])$ by

$$
\exp (T[\underline{l}])=\sum_{k=0}^{\infty} \frac{1}{k !} T[\underline{l}]^{k} .
$$


Then, $\exp (T[\underline{l}])$ operates on $\mathcal{F}$ from the left and on $\mathcal{F}^{\dagger}$ from the right.

By Lemma 6.1, for any automorphism $h \in \mathcal{D}_{+}^{0}$, there exist a unique $\underline{l} \in \underline{d}_{+}^{0}$ with $\exp (\underline{l})=h$. Now for $h \in \mathcal{D}_{+}^{0}$ define the operator $G[h]$ by

$$
G[h]=\exp (-T[\underline{l}])
$$

where $\exp (\underline{l})=h$. Then, $G[h]$ operates on $\mathcal{F}$ from the left and on $\mathcal{F}^{\dagger}$ from the right. Then we have the following important results.

Theorem 6.1. For any $h \in \mathcal{D}_{+}^{0}, f(\xi) d \xi \in \mathbf{C}((\xi)) d \xi, g(\xi) \in \mathbf{C}((\xi))$ and $\underline{l}=l(\xi) \frac{d}{d \xi} \in$ $\mathbf{C}((\xi)) \frac{d}{d \xi}$, we have the following equalities as operators on $\mathcal{F}$ and $\mathcal{F}^{\dagger}$.

$$
\begin{aligned}
G[h](\psi[f(\xi) d \xi]) G[h]^{-1} & =\psi\left[h^{*}(f(\xi) d \xi)\right]=\psi\left[f(h(\xi)) h^{\prime}(\xi) d \xi\right] \\
G[h](\bar{\psi}[g(\xi)]) G[h]^{-1} & =\bar{\psi}\left[h^{*}(g(\xi))\right]=\bar{\psi}[g(h(\xi))] \\
G\left[h_{1} \circ h_{2}\right] & =G\left[h_{1}\right] G\left[h_{2}\right] \\
G[h] T[\underline{l}] G[h]^{-1} & =T[\operatorname{ad}(h)(\underline{l})]+\frac{1}{6} \operatorname{Res}_{\xi=0}(\{h(\xi) ; \xi\} l(\xi) d \xi) .
\end{aligned}
$$

where $\{f(\xi) ; \xi\}$ is the Schwarzian derivative.

A proof is easily given by applying (5.3) and (5.4). From this theorem we infer easily the following proposition.

Proposition 6.1. For any $h_{j} \in \mathcal{D}_{+}^{0}, j=1,2, \ldots, N$ and $N$-pointed curve

$$
\mathfrak{X}=\left(C ; Q_{1}, Q_{2}, \ldots, Q_{n} ; \xi_{1}, \xi_{2}, \ldots, \xi_{N}\right)
$$

with formal coordinates, put

$$
\mathfrak{X}_{(h)}=\left(C ; Q_{1}, Q_{2}, \ldots, Q_{N} ; h_{1}\left(\xi_{1}\right), h_{2}\left(\xi_{2}\right), \ldots, h_{N}\left(\xi_{N}\right)\right) .
$$

Then, the isomorphism $G\left[h_{1}\right] \widehat{\otimes} \cdots \widehat{\otimes} G\left[h_{N}\right]$

$$
\begin{aligned}
\mathcal{F}_{N}^{\dagger} & \rightarrow \mathcal{F}_{N}^{\dagger} \\
\left\langle\phi_{1} \widehat{\otimes} \cdots \widehat{\otimes} \phi_{N}\right| & \mapsto\left\langle\phi_{1} G\left[h_{1}\right] \widehat{\otimes} \cdots \widehat{\otimes} \phi_{N} G\left[h_{N}\right]\right|
\end{aligned}
$$

induces the canonical isomorphism

$$
G\left[h_{1}\right] \widehat{\otimes} \cdots \widehat{\otimes} G\left[h_{N}\right]: \mathcal{V}^{\dagger}{ }_{\mathrm{ab}}(\mathfrak{X}) \rightarrow \mathcal{V}^{\dagger}{ }_{\mathrm{ab}}\left(\mathfrak{X}_{(h)}\right)
$$

Let $\mathfrak{X}=(C ; Q ; \xi)$ be a one-pointed smooth curve of genus $g$ with a formal coordinate. We shall show that if we fix a symplectic basis $\left\{\alpha_{1}, \ldots, \alpha_{g}, \beta_{1}, \ldots, \beta_{g}\right\}$ of $H_{1}(C, \mathbf{Z})$, then there is a canonical preferred non-zero vector $\langle\omega(\mathfrak{X},\{\alpha, \beta\})| \in \mathcal{V}^{\dagger}{ }_{a b}(\mathfrak{X})$ which is a refinement of the construction given in Lemma 3.1. Let us choose a normalized basis $\left\{\omega_{1}, \ldots, \omega_{g}\right\}$ of holomorphic one-forms on $C$ which is characterized by

$$
\int_{\beta_{i}} \omega_{j}=\delta_{i j}, \quad 1 \leq i, j \leq g
$$


The matrix

$$
\tau=\left(\tau_{i j}\right), \quad \tau_{i j}=\int_{\alpha_{i}} \omega_{j}
$$

is then called the period matrix of the curve $C$. Now the numbers $I_{n}^{i}, n=1,2, \ldots$, $i=1, \ldots g$ are defined by

$$
\omega_{i}=\left(\sum_{n=1}^{\infty} I_{n}^{i} \xi^{n-1}\right) d \xi
$$

Note that the numbers $I_{n}^{i}$ depend on the symplectic basis $\left\{\alpha_{1}, \ldots, \alpha_{g}, \beta_{1}, \ldots, \beta_{g}\right\}$ and the coordinate $\xi$.

For a positive integer $n \geq 1$ let $\omega_{Q}^{(n)}$ be a meromorphic one-form on $C$ which has a pole of order $n+1$ at $Q$ and holomorphic elsewhere such that

$$
\begin{aligned}
\int_{\alpha_{i}} \omega_{Q}^{(n)} & =-\frac{2 \pi \sqrt{-1} I_{n}^{i}}{n}, \quad \int_{\beta_{i}} \omega_{Q}^{(n)}=0, \quad 1 \leq i \leq g \\
\omega_{Q}^{(n)} & =\left(\frac{1}{\xi^{n+1}}+\sum_{m=1}^{\infty} q_{n, m} \xi^{m-1}\right) d \xi .
\end{aligned}
$$

These conditions uniquely determine $\omega_{Q}^{(n)}$. Note that the second equality of (6.2) and (6.3) imply the first equality of (6.2). The preferred element $\langle\omega(\mathfrak{X},\{\alpha, \beta\})| \in \mathcal{V}^{\dagger}{ }_{\mathrm{ab}}(\mathfrak{X})$ is defined by

$$
\langle\omega(\mathfrak{X},\{\alpha, \beta\})|=\cdots e\left(\omega_{g+2}\right) \wedge e\left(\omega_{g+1}\right) \wedge e\left(\omega_{g}\right) \wedge \cdots \wedge e\left(\omega_{1}\right),
$$

where

$$
\omega_{g+n}=\omega_{Q}^{(n)} .
$$

For details see Lemma 3.1. We call $\left\{\omega_{n}\right\}, n=1,2, \ldots$ a normalized basis for $\mathfrak{X}$. Note that the normalized basis depends on the choice of a symplectic basis of $H_{1}(C, \mathbf{Z})$ and the coordinate $\xi$.

Theorem 6.2. For $h(\xi) \in \mathcal{D}_{+}^{0}$ put $\mathfrak{X}_{h}=\{C ; Q ; \eta=h(\xi)\}$. Then

$$
\langle\omega(\mathfrak{X},\{\alpha, \beta\})| G[h]=\left\langle\omega\left(\mathfrak{X}_{h},\{\alpha, \beta\}\right)\right|,
$$

where $G[h]: \mathcal{V}^{\dagger}\left(\mathfrak{X}_{h}\right) \rightarrow \mathcal{V}^{\dagger}(\mathfrak{X})$ is the canonical isomorphism given in Proposition 6.1

Proof. $\quad$ First consider the case in which $h(\xi)=a \xi$ for a positive number $a$. Put $\eta=a \xi$. Let $\omega_{i}, i=1, \ldots, g$ and $\omega_{g+n}=\omega_{Q}^{(n)}$ be chosen for $\mathfrak{X}$ as above. Then

$$
\begin{aligned}
\omega_{i} & =\left(\sum_{n=1}^{\infty} a^{-n-1} I_{n}^{i} \eta^{n-1}\right) d \eta, \\
\omega_{Q}^{(n)} & =\left(\frac{a^{n}}{\eta^{n+1}}+\sum_{m=1}^{\infty} a^{-m-1} q_{n, m} \eta^{m-1}\right) d \eta .
\end{aligned}
$$

Put

$$
\begin{aligned}
\widetilde{\omega}_{i} & =\omega_{i}, \quad 1 \leq i \leq g \\
\widetilde{\omega}_{Q}^{(n)} & =a^{-n} \omega_{Q}^{(n)}=\left(\frac{1}{\eta^{n+1}}+\sum_{m=1}^{\infty} \frac{q_{n, m}}{a^{-n-m-1}} \eta^{m-1}\right) d \eta .
\end{aligned}
$$


Then, the element $\left\langle\mathfrak{X}_{h},\{\alpha, \beta\}\right| \in \mathcal{V}^{\dagger}{ }_{\mathrm{ab}}\left(\mathfrak{X}_{h}\right)$ is given by

$$
\langle\widetilde{\omega}(\mathfrak{X},\{\alpha, \beta\})|=\cdots e\left(\widetilde{\omega}_{g+2}\right) \wedge e\left(\widetilde{\omega}_{g+1}\right) \wedge e\left(\widetilde{\omega}_{g}\right) \wedge \cdots e\left(\widetilde{\omega}_{1}\right),
$$

where

$$
\widetilde{\omega}_{g+n}=\widetilde{\omega}_{Q}^{(n)} .
$$

Note that $\langle\omega(\mathfrak{X},\{\alpha, \beta\})|,\langle\widetilde{\omega}(\mathfrak{X},\{\alpha, \beta\})| \in \mathcal{F}(g-1)$.

Let $\alpha$ be the positive number such that $\exp (\alpha)=a$. Put

$$
\underline{l}=\alpha \xi \frac{d}{d \xi}
$$

Then we have

$$
T[\underline{l}]=\alpha L_{0}, \quad G[h]=\exp \left(-\alpha L_{0}\right) .
$$

On $\mathcal{F}_{d}^{\dagger}(g-1), G[h]$ operates by the multiplication by $a^{-(d+g(g-1) / 2)}$. The coefficient of $\cdots e^{-l_{1}-5 / 2} \wedge e^{-l_{1}-3 / 2} \wedge e^{m_{1}+1 / 2} \wedge e^{-l_{1}+1 / 2} \wedge \cdots \wedge e^{m_{k}+1 / 2} \wedge \cdots \wedge e^{-3 / 2} \wedge e^{n_{g}+1 / 2} \wedge \cdots \wedge e^{n_{1}+1 / 2}$

in $\langle\omega(\mathfrak{X},\{\alpha, \beta\})|$ is

$$
A=q_{l_{1}, m_{1}+1} \cdots q_{l_{k}, m_{k}+1} I_{n_{g}+1}^{g} \cdots I_{n_{1}+1}^{1} .
$$

The degree $d$ of this term is given by formula (5.6)

$$
d=\sum_{i=1}^{g} n_{i}+\sum_{j=1}^{l}\left(m_{j}+l_{j}+1\right)-\frac{g(g-1)}{2} .
$$

Thus we have that

$$
-d-g(g-1) / 2=-\sum_{i=1}^{g} n_{i}-\sum_{j=1}^{l}\left(m_{j}+l_{j}+1\right) .
$$

On the other hand the coefficient of $\left\langle\omega\left(\mathfrak{X}_{h},\{\alpha, \beta\}\right)\right|$ is

$$
a^{-\sum_{i=1}^{g} n_{i}-\sum_{j=1}^{l}\left(m_{j}+l_{j}+1\right)} A=a^{-(d+g(g-1) / 2)} A .
$$

This implies

$$
\langle\omega(\mathfrak{X},\{\alpha, \beta\})| G[h]=\left\langle\omega\left(\mathfrak{X}_{h},\{\alpha, \beta\}\right)\right| .
$$

Now let us consider the case in which $h(\xi)$ is an element of $\mathcal{D}^{1} \subset \mathbf{C}((\xi))$. Let $\left\{\widetilde{\omega}_{n}\right\}$, $n=1,2, \ldots$ be a normalized basis of $\mathfrak{X}_{h}$. Put

$$
\omega_{n}=h^{*}\left(\widetilde{\omega}_{n}\right) .
$$

Since $h(\xi)=\xi+a_{1} \xi^{2}+\cdots$, the normalized basis of $\langle\mathfrak{X}|$ is given by $\left\{\omega_{n}\right\}, n=1,2, \ldots$ For any positive integer $m$ all terms in

$$
\left\langle\phi_{m}\right|=\cdots e^{-m-5 / 2} \wedge e^{-m-3 / 2} \wedge e\left(\omega_{m+g}\right) \wedge \cdots \wedge e\left(\omega_{1}\right)
$$


are also present in $\langle\omega(\mathfrak{X},\{\alpha, \beta\})|$ by its definition. Moreover, we can express

$$
\begin{aligned}
\left\langle\phi_{m}\right| & =\langle-m-1| \psi\left[\omega_{m+g}\right] \cdots \psi\left[\omega_{1}\right] \\
& =\langle-m-1| \psi\left[h^{*}\left(\widetilde{\omega}_{m+g}\right)\right] \cdots \psi\left[h^{*}\left(\widetilde{\omega}_{1}\right)\right] .
\end{aligned}
$$

Then by Theorem 6.1

$$
\left\langle\phi_{m}\right|=\langle-m-1| G[h] \psi\left[\widetilde{\omega}_{m+g}\right] \cdots \psi\left[\widetilde{\omega}_{1}\right] G[h]^{-1} .
$$

Note that

$$
\langle-m-1| \equiv\langle-m-1| \bmod \mathcal{F}_{1}(-m-1) .
$$

It is easy to show that each term appearing in

$$
\left\langle\phi_{m}\right| G[h]-\langle-m-1| \psi\left[\widetilde{\omega}_{m+g}\right] \cdots \psi\left[\widetilde{\omega}_{1}\right] G[h]^{-1} .
$$

does not appear in

$$
\left\langle\phi_{m+1}\right|=\langle-m-2| G[h] \psi\left[\widetilde{\omega}_{m+1+g}\right] \cdots \psi\left[\widetilde{\omega}_{1}\right] G[h]^{-1} .
$$

Taking the limit $m \rightarrow \infty$ we conclude

$$
\langle\omega(\mathfrak{X},\{\alpha, \beta\})|=\left\langle\omega\left(\mathfrak{X}_{h},\{\alpha, \beta\}\right)\right| G[h]^{-1} .
$$

Next let us study the dependence of the section $\langle\omega(\mathfrak{X},\{\alpha, \beta\})|$ on the choice of symplectic basis.

Theorem 6.3. Let $\left\{\alpha_{1}, \ldots \alpha_{g}, \beta_{1}, \ldots, \beta_{g}\right\}$ and $\left\{\widetilde{\alpha}_{1}, \ldots \widetilde{\alpha}_{g}, \widetilde{\beta}_{1}, \ldots, \widetilde{\beta}_{g}\right\}$ be symplectic bases of $H^{1}(C, \mathbf{Z})$ of the non-singular curve $C$. Assume that $\left\{\beta_{1}, \ldots, \beta_{g}\right\}$ and $\left\{\widetilde{\beta}_{1}, \ldots, \widetilde{\beta}_{g}\right\}$ span the same Lagrangian sublattice in $H^{1}(C, \mathbf{Z})$. Then

$$
\langle\omega(\mathfrak{X},\{\alpha, \beta\})|=\operatorname{det} U\langle\omega(\mathfrak{X},\{\widetilde{\alpha}, \widetilde{\beta}\})|,
$$

where $U \in G L(g, \mathbf{Z})$ is defined by

$$
\left(\begin{array}{c}
\widetilde{\beta}_{1} \\
\vdots \\
\widetilde{\beta}_{g}
\end{array}\right)=U\left(\begin{array}{c}
\beta_{1} \\
\vdots \\
\beta_{g}
\end{array}\right) .
$$

Proof. By the assumption on the $\beta$-cycles we have that

$$
\left(\begin{array}{c}
\widetilde{\alpha} \\
\widetilde{\beta}
\end{array}\right)=\left(\begin{array}{cc}
{ }^{t} U^{-1} & B \\
0 & U
\end{array}\right)\left(\begin{array}{c}
\alpha \\
\beta
\end{array}\right)
$$

where $B$ is a $g \times g$ integral matrix. For a normalized basis $\left\{\omega_{1}, \ldots, \omega_{g}\right\}$ of holomorphic one-forms with respect to the symplectic basis $\{\alpha, \beta\}$ we have that

$$
\left(\int_{\widetilde{\beta}_{i}} \omega_{j}\right)=U
$$


Hence the normalized basis of holomorphic one-forms $\left\{\widetilde{\omega}_{1}, \ldots, \widetilde{\omega_{g}}\right\}$ with respect to $\{\widetilde{\alpha}, \widetilde{\beta}\}$ is given by

$$
\left(\widetilde{\omega}_{1}, \ldots, \widetilde{\omega_{g}}\right)=\left(\omega_{1}, \ldots, \omega_{g}\right) U^{-1} .
$$

Thus

$$
\left(\widetilde{I}_{n}^{1}, \ldots, \widetilde{I}_{n}^{g}\right)=\left(I_{n}^{1}, \ldots, I_{n}^{g}\right) U^{-1},
$$

for all $n$ 's. Therefore, for the normalized meromorphic one-form $\omega_{Q}^{(n)}$ with respect to the symplectic basis $\{\alpha, \beta\}$ we have that

$$
\begin{aligned}
&\left(\begin{array}{c}
\int_{\widetilde{\alpha}_{1}} \omega_{Q}^{(n)} \\
\vdots \\
\int_{\widetilde{\alpha}_{g}} \omega_{Q}^{(n)}
\end{array}\right)={ }^{t} U^{-1}\left(\begin{array}{c}
\int_{\alpha_{1}} \omega_{Q}^{(n)} \\
\vdots \\
\int_{\widetilde{\alpha}_{g}} \omega_{Q}^{(n)}
\end{array}\right)+B\left(\begin{array}{c}
\int_{\beta_{1}} \omega_{Q}^{(n)} \\
\vdots \\
\int_{\beta_{g}} \omega_{Q}^{(n)}
\end{array}\right) \\
&={ }^{t} U^{-1}\left(\begin{array}{c}
I_{n}^{1} \\
\vdots \\
I_{n}^{g}
\end{array}\right)=\left(\begin{array}{c}
\widetilde{I}_{n}^{1} \\
\vdots \\
\widetilde{I}_{n}^{g}
\end{array}\right) \\
&\left(\begin{array}{c}
\int_{\widetilde{\beta}_{1}} \omega_{Q}^{(n)} \\
\vdots \\
\int_{\widetilde{\beta}_{g}} \omega_{Q}^{(n)}
\end{array}\right)=U\left(\begin{array}{c}
\int_{\beta_{1}} \omega_{Q}^{(n)} \\
\vdots \\
\int_{\beta_{g}} \omega_{Q}^{(n)}
\end{array}\right)=0
\end{aligned}
$$

Thus $\omega_{Q}^{(n)}$ is also the normalized meromorphic one-form with respect to the symplectic basis $\{\widetilde{\alpha}, \widetilde{\beta}\}$. Hence we have that

$$
\langle\omega(\mathfrak{X},\{\alpha, \beta\})|=\operatorname{det} U\langle\omega(\mathfrak{X},\{\widetilde{\alpha}, \widetilde{\beta}\})| .
$$

Note that $\operatorname{det} U= \pm 1$. Next we shall show that $\langle\omega(\mathfrak{X})|$ is independent of the point on the curve $C$. For that purpose we need the following lemma. We use the basis $\left\{\omega_{1}, \ldots, \omega_{g}\right\}$ of holomorphic one-forms on the curve $C$ normalized by (6.1).

Lemma 6.2. The numbers $1=n_{1}<n_{2}<\cdots<n_{g} \leq 2 g-1$ are the Weierstrass gap values if and only if

$$
\operatorname{det}\left|\begin{array}{ccc}
I_{n_{1}}^{1} & \cdots & I_{n_{g}}^{g} \\
\vdots & \ddots & \vdots \\
I_{n_{g}}^{1} & \cdots & I_{n_{g}}^{g}
\end{array}\right| \neq 0
$$

Proof. If

$$
\operatorname{det}\left|\begin{array}{ccc}
I_{n_{1}}^{1} & \cdots & I_{n_{g}}^{g} \\
\vdots & \ddots & \vdots \\
I_{n_{g}}^{1} & \cdots & I_{n_{g}}^{g}
\end{array}\right|=0
$$

then there exists a vector $\left(a_{1}, \ldots, a_{g}\right) \neq(0, \ldots, 0)$ such that

$$
\left(a_{1}, \ldots, a_{g}\right)\left(\begin{array}{ccc}
I_{n_{1}}^{1} & \cdots & I_{n_{g}}^{g} \\
\vdots & \ddots & \vdots \\
I_{n_{g}}^{1} & \cdots & I_{n_{g}}^{g}
\end{array}\right)=(0, \ldots, 0) .
$$


Form this we infer

$$
\operatorname{Res}_{\xi=0}\left(\left(a_{g} \xi^{-n_{g}}+\cdots+a_{1} \xi^{-n_{1}}\right) \omega_{i}\right)=0, \quad i=1, \ldots, g .
$$

This means that there is a meromorphic function $f \in H^{0}\left(C, \mathcal{O}_{C}(* Q)\right)$ whose principal part at the point $Q$ is $a_{g} \xi^{-n_{g}}+\cdots+a_{1} \xi^{-n_{1}}$. Thus $n_{g}$ is not a Weierstrass gap value.

On the other hand if

$$
\operatorname{det}\left|\begin{array}{ccc}
I_{n_{1}}^{1} & \cdots & I_{n_{1}}^{g} \\
\vdots & \ddots & \vdots \\
I_{n_{g}}^{1} & \cdots & I_{n_{g}}^{g}
\end{array}\right| \neq 0
$$

for any vector $\left(a_{1}, \ldots, a_{g}\right) \neq 0$, we have

$$
\left(a_{1}, \ldots, a_{g}\right)\left(\begin{array}{ccc}
I_{n_{1}}^{1} & \cdots & I_{n_{1}}^{g} \\
\vdots & \ddots & \vdots \\
I_{n_{g}}^{1} & \cdots & I_{n_{g}}^{g}
\end{array}\right) \neq(0, \ldots, 0)
$$

This means that

$$
\left.\operatorname{Res}_{\xi=0}\left(\left(a_{g} \xi^{-n_{g}}+\cdots+a_{1} \xi^{-n_{1}}\right) \omega_{i}\right)\right) \neq 0,
$$

for a suitable $1 \leq i \leq g$. Hence there does not exists a meromorphic function $f \in$ $H^{0}\left(C, \mathcal{O}_{C}(* Q)\right)$ whose principal part at $Q$ is $a_{g} \xi^{-n_{g}}+\cdots+a_{1} \xi^{-n_{1}}$.

QED

Let $\{P, Q\}$ be two smooth points on the curve $C$ with formal coordinates $\xi, \eta$, respectively. Put $\mathfrak{X}_{0}=(C ; P, Q ; \xi, \eta), \mathfrak{X}_{1}=(C ; P ; \xi), \mathfrak{X}_{2}=(C ; Q ; \eta)$. Then the natural imbeddings

$$
\begin{aligned}
& \iota_{1} \quad: \quad \mathcal{F} \hookrightarrow \mathcal{F}_{2} \\
&|u\rangle \mapsto|u\rangle \otimes|0\rangle \\
& \iota_{2} \quad: \quad \mathcal{F} \hookrightarrow \mathcal{F}_{2} \\
& \quad|u\rangle \mapsto|0\rangle \otimes|u\rangle
\end{aligned}
$$

induce canonical isomorphisms

$$
\begin{array}{cc} 
& \mathcal{V}^{\dagger}{ }_{\mathrm{ab}}\left(\mathfrak{X}_{0}\right) \\
\iota_{1}^{*} \swarrow & \searrow^{\iota_{2}^{*}} \\
\mathcal{V}^{\dagger}{ }_{\mathrm{ab}}\left(\mathfrak{X}_{1}\right) & \mathcal{V}_{\mathrm{ab}}^{\dagger}\left(\mathfrak{X}_{2}\right)
\end{array}
$$

by Theorem 3.4.

Theorem 6.4. Under the above notation we have

$$
\iota_{2}^{*} \circ\left(\iota_{1}^{*}\right)^{-1}\left(\left\langle\omega\left(\mathfrak{X}_{1},\{\alpha, \beta\}\right)\right|\right)=\left\langle\omega\left(\mathfrak{X}_{2},\{\alpha, \beta\}\right)\right| .
$$


Proof. $\quad$ Put $\langle\widetilde{\Phi}|=\left(\iota_{1}^{*}\right)^{-1}\left(\left\langle\omega\left(\mathfrak{X}_{1},\{\alpha, \beta\}\right)\right|\right)$. Then, by Theorem 3.4 we have

$$
\langle\widetilde{\Phi} \mid u \otimes 0\rangle=\left\langle\omega\left(\mathfrak{X}_{1},\{\alpha, \beta\}\right) \mid u\right\rangle
$$

for any $|u\rangle \in \mathcal{F}$. Since the space of ghost vacua is one-dimensional, we have that

$$
\langle\widetilde{\Phi} \mid 0 \otimes u\rangle=c\left\langle\omega\left(\mathfrak{X}_{2},\{\alpha, \beta\}\right) \mid u\right\rangle
$$

for a constant independent of $|u\rangle \in \mathcal{F}$. To determine $c$ it is enough to choose a $|u\rangle$ such that we can calculate both sides of the equality (6.6). Let $1=n_{1}<n_{2}<\cdots<n_{g}$ be the gap values at the point $Q$ and put

$$
|u\rangle=\bar{e}_{n_{g}-1 / 2} \wedge \cdots \bar{e}_{n_{1}-1 / 2} \wedge|-1\rangle .
$$

Let $f_{j} \in H^{0}\left(C, \mathcal{O}_{C}(*(P+Q)), j=1,2, \ldots, g\right.$ be chosen in such a way that $f_{j}$ has the Laurent expansion

$$
f_{j}=\eta^{-n_{j}}+a_{0}^{(j)}+a_{1}^{(j)} \eta+\cdots
$$

at $Q$. Also let $\omega_{Q, P}$ be a meromorphic one-form having poles of order one at $Q$ with residue 1 and at $P$ with residue -1 and else holomorphic. Then we have that

$$
\begin{aligned}
\langle\widetilde{\Phi} \mid 0 \otimes u\rangle & =\left\langle\widetilde{\Phi}\left|0 \otimes \rho_{Q}\left(\bar{\psi}\left[f_{g}\right]\right) \rho_{Q}\left(\bar{\psi}\left[f_{g-1}\right]\right) \cdots \rho_{Q}\left(\bar{\psi}\left[f_{1}\right]\right)\right|-1\right\rangle \\
& =\left\langle\widetilde{\Phi} \mid 0 \otimes \rho_{Q}\left(\bar{\psi}\left[f_{g}\right]\right) \rho_{Q}\left(\bar{\psi}\left[f_{g-1}\right]\right) \cdots \rho_{Q}\left(\bar{\psi}\left[f_{1}\right]\right) \rho_{Q}\left(\psi\left[\omega_{Q, P}\right]\right) 0\right\rangle \\
& =(-1)^{g}\left\langle\widetilde{\Phi} \mid 0 \otimes \rho_{Q}\left(\psi\left[\omega_{Q, P}\right]\right) \rho_{Q}\left(\bar{\psi}\left[f_{g}\right]\right) \rho_{Q}\left(\bar{\psi}\left[f_{g-1}\right]\right) \cdots \rho_{Q}\left(\bar{\psi}\left[f_{1}\right]\right) 0\right\rangle \\
& =(-1)^{g+1}\left\langle\widetilde{\Phi} \mid \rho_{P}\left(\bar{\psi}\left[\omega_{Q, P}\right]\right) 0 \otimes \rho_{Q}\left(\bar{\psi}\left[f_{g}\right]\right) \rho_{Q}\left(\bar{\psi}\left[f_{g-1}\right]\right) \cdots \rho_{Q}\left(\bar{\psi}\left[f_{1}\right]\right) 0\right\rangle \\
& =(-1)^{g}\left\langle\widetilde{\Phi} \mid-1 \otimes \rho_{Q}\left(\bar{\psi}\left[f_{g}\right]\right) \rho_{Q}\left(\bar{\psi}\left[f_{g-1}\right]\right) \cdots \rho_{Q}\left(\bar{\psi}\left[f_{1}\right]\right) 0\right\rangle \\
& =(-1)^{g}\left\langle\widetilde{\Phi}\left|\rho_{P}\left(\bar{\psi}\left[f_{g}\right]\right)\right|-1 \otimes \rho_{Q}\left(\bar{\psi}\left[f_{g-1}\right]\right) \cdots \rho_{Q}\left(\bar{\psi}\left[f_{1}\right]\right) 0\right\rangle \\
& =(-1)^{g+g(g-1) / 2}\left\langle\widetilde{\Phi}\left|\rho_{P}\left(\bar{\psi}\left[f_{1}\right]\right) \cdots \rho_{P}\left(\bar{\psi}\left[f_{g-1}\right]\right) \rho_{P}\left(\bar{\psi}\left[f_{g}\right]\right)\right|-1 \otimes 0\right\rangle \\
& =(-1)^{g}\left\langle\widetilde{\Phi}\left|\rho_{P}\left(\bar{\psi}\left[f_{g}\right]\right) \rho_{P}\left(\bar{\psi}\left[f_{g-1}\right]\right) \cdots \rho_{P}\left(\bar{\psi}\left[f_{1}\right]\right)\right|-1 \otimes 0\right\rangle \\
& =(-1)^{g}\left\langle\omega\left(\mathfrak{X}_{1},\{\alpha, \beta\}\right)\left|\rho_{P}\left(\bar{\psi}\left[f_{g}\right]\right) \rho_{P}\left(\bar{\psi}\left[f_{g-1}\right]\right) \cdots \rho_{P}\left(\bar{\psi}\left[f_{1}\right]\right)\right|-1\right\rangle
\end{aligned}
$$

The last term can be expressed in a matrix form

$$
(-1)^{g}\left|\begin{array}{cccc}
\operatorname{Res}_{P}\left(f_{g} \omega_{1}\right) & \operatorname{Res}_{P}\left(f_{g} \omega_{2}\right) & \cdots & \operatorname{Res}_{P}\left(f_{g} \omega_{g}\right) \\
\operatorname{Res} P\left(f_{g-1} \omega_{1}\right) & \operatorname{Res}_{P}\left(f_{g-1} \omega_{2}\right) & \cdots & \operatorname{Res}_{P}\left(f_{g-1} \omega_{g}\right) \\
\vdots & \vdots & \ddots & \vdots \\
\operatorname{Res}_{P}\left(f_{2} \omega_{1}\right) & \operatorname{Res}_{P}\left(f_{2} \omega_{2}\right) & \cdots & \operatorname{Res}_{P}\left(f_{2} \omega_{g}\right) \\
\operatorname{Res}_{P}\left(f_{1} \omega_{1}\right) & \operatorname{Res}_{P}\left(f_{1} \omega_{2}\right) & \cdots & \operatorname{Res}_{P}\left(f_{1} \omega_{g}\right)
\end{array}\right|
$$

But $f_{j} \omega_{k}$ has poles only at $P$ and $Q$, thus

$$
\operatorname{Res}_{P}\left(f_{j} \omega_{k}\right)=-\operatorname{Res}_{Q}\left(f_{j} \omega_{k}\right)=-I_{n_{j}}^{k}(Q)
$$

where

$$
\omega_{k}=\sum_{n=1}^{\infty} I_{n}^{k}(Q) \eta^{n-1} d \eta
$$


Hence the above determinant is

$$
\left|\begin{array}{ccc}
I_{n_{g}}^{1}(Q) & \cdots & I_{n_{g}}^{g}(Q) \\
\vdots & \ddots & \vdots \\
I_{n_{1}}^{1}(Q) & \cdots & I_{n_{1}}^{g}(Q)
\end{array}\right|=\left\langle\omega\left(\mathfrak{X}_{2},\{\alpha, \beta\}\right)\left|\bar{e}_{n_{g}-1 / 2} \wedge \cdots \wedge \bar{e}_{n_{1}-1 / 2} \wedge\right|-1\right\rangle
$$

Hence we have that

$$
\left\langle\widetilde{\Phi}\left|0 \otimes \bar{e}_{n_{g}-1 / 2} \wedge \cdots \wedge \bar{e}_{n_{1}-1 / 2} \wedge\right|-1\right\rangle=\left\langle\omega\left(\mathfrak{X}_{2},\{\alpha, \beta\}\right)\left|\bar{e}_{n_{g}-1 / 2} \wedge \cdots \wedge \bar{e}_{n_{1}-1 / 2} \wedge\right|-1\right\rangle .
$$

This shows $c=1$.

QED

Let us consider a curve $C$ with a node $P$. Let $\widetilde{C}$ be the curve obtained by resolving the singularity at $P$ and let $\pi: \widetilde{C} \rightarrow C$ be the natural holomorphic mapping. Then $\pi^{-1}(P)$ consists of two points $P_{+}$and $P_{-}$. Assume that $\widetilde{C}$ is connected.

Let

$$
\mathfrak{X}=(C ; Q ; \xi)
$$

be a one-pointed curve with formal coordinates and we let

$$
\widetilde{\mathfrak{X}}=\left(\widetilde{C} ; P_{+}, P_{-}, Q ; z, w, \xi\right)
$$

be the associated 3-pointed curve with formal coordinates. Then Theorem 3.5 says that the natural isomorphism

$$
\left|0_{+,-}\right\rangle \otimes \mathcal{F} \cong \mathcal{F}
$$

induces the natural isomorphism

$$
\iota_{+,-}^{*}: \mathcal{V}^{\dagger}{ }_{\mathrm{ab}}(\widetilde{\mathfrak{X}}) \cong \mathcal{V}_{\mathrm{ab}}^{\dagger}(\mathfrak{X}) .
$$

where

$$
\left|0_{+,-}\right\rangle=|0\rangle \otimes|-1\rangle-|-1\rangle \otimes|0\rangle .
$$

We can define $\langle\omega(\mathfrak{X},\{\alpha, \beta\})|$ similar to the non-singular case, by choosing a basis $\{\alpha, \beta\}=$ $\left\{\alpha_{1}, \ldots, \alpha_{g-1}, \alpha_{g}, \beta_{1}, \ldots, \beta_{g-1}\right\}$ of $H_{1}(C, \mathbf{Z})$, in such a way that $\alpha_{1}, \alpha_{2}, \ldots, \alpha_{g-1}$ and $\beta_{1}, \beta_{2}, \ldots, \beta_{g-1}$ is the image of a symplectic basis of $H_{1}(\widetilde{C}, \mathbf{Z})$ under natural map to $H_{1}(C, \mathbf{Z})$ and $\alpha_{g}$ corresponds to the invariant cycle of a flat deformation of the curve $C$. Then we can choose a basis $\left\{\omega_{1}, \ldots, \omega_{g-1}, \omega_{g}, \omega_{g+1}, \omega_{g+2}, \ldots\right\}$ of $H^{0}(C, \omega(* Q))$ such that $\left\{\pi^{*} \omega_{1}, \ldots, \pi^{*} \omega_{g-1}, \pi^{*} \omega_{g+1}, \pi^{*} \omega_{g+2}, \ldots\right\}$ is a normalized basis of $H^{0}\left(\widetilde{C}, \omega_{\widetilde{C}}(* Q)\right)$ as in (6.1), (6.2) and (6.3) where we put

$$
\pi^{*} \omega_{g+n}=\omega_{Q}^{(n)}, \quad n=1,2, \ldots,
$$

and $\pi^{*} \omega_{g}$ is a meromorphic one-form on $\widetilde{C}$ which has poles of order one at $P_{+}$and $P_{-}$ with residue -1 and 1 , respectively, is holomorphic outside $P_{ \pm}$and

$$
\int_{P_{+}}^{P_{-}} \pi^{*} \omega_{g}=1
$$

Then put

$$
\langle\omega(\mathfrak{X},\{\alpha, \beta\})|=\left\langle\cdots \wedge e\left(\omega_{m}\right) \wedge \cdots \wedge e\left(\omega_{2}\right) \wedge e\left(\omega_{1}\right)\right| .
$$


The proof of Lemma 3.1 applies also in this case and shows that $\langle\omega(\mathfrak{X},\{\alpha, \beta\})|$ is an element of $\mathcal{V}^{\dagger}{ }_{\mathrm{ab}}(\mathfrak{X})$. Let

$$
\widehat{\mathfrak{X}}=(\widetilde{C}, Q ; \xi) .
$$

Then, by applying Theorem 3.4 at the points $P_{ \pm}$we have a canonical isomorphism

$$
\iota^{*}: \mathcal{V}_{\mathrm{ab}}^{\dagger}(\widetilde{\mathfrak{X}}) \cong \mathcal{V}_{\mathrm{ab}}^{\dagger}(\widehat{\mathfrak{X}}) .
$$

Theorem 6.5. Under the above assumptions and notation we have that

$$
\iota^{*} \circ\left(\iota_{+,-}^{*}\right)^{-1}(\langle\omega(\mathfrak{X},\{\alpha, \beta\})|)=(-1)^{g}\langle\omega(\widehat{\mathfrak{X}},\{\widehat{\alpha}, \widehat{\beta}\})|
$$

where $\{\widehat{\alpha}, \widehat{\beta}\}=\left\{\alpha_{1}, \ldots, \alpha_{g-1}, \beta_{1}, \ldots, \beta_{g-1}\right\}$.

Proof. Put

$$
\langle\widetilde{\Phi}|=\left(\iota_{+,-}^{*}\right)^{-1}(\langle\omega(\mathfrak{X},\{\alpha, \beta\})|) .
$$

By the definition of $\iota^{*}$ we have that

$$
\langle\widetilde{\Phi} \mid 0 \otimes 0 \otimes u\rangle=\left\langle\iota^{*} \circ\left(\iota_{+,-}^{*}\right)^{-1}(\langle\omega(\mathfrak{X},\{\alpha, \beta\})|) \mid u\right\rangle .
$$

Choose a meromorphic function $f \in H^{0}\left(\widetilde{C}, \mathcal{O}_{\widetilde{C}}(* Q)\right)$ such that $f\left(P_{+}\right)=-1$ and $f\left(P_{-}\right)=$ 0 . Then by (3.18) (using the notation $\langle\omega(\mathfrak{X})|=\langle\omega(\mathfrak{X},\{\alpha, \beta\})|$ ), we have that

$$
\langle\widetilde{\Phi} \mid 0 \otimes 0 \otimes u\rangle=\left\langle\omega(\mathfrak{X}) \mid \rho_{Q}(\bar{\psi}[f]) u\right\rangle
$$

for any $|u\rangle \in \mathcal{F}$. The proof of lemma 3.1 show that for a meromorphic form $\omega_{j}$ on $C$ we have that

$$
\wedge e\left(\omega_{j}\right) \bar{\psi}[f]=\operatorname{Res}_{Q}\left(f \omega_{j}\right)=\operatorname{Res}_{Q}\left(f \pi^{*} \omega_{j}\right)=\wedge e\left(\pi^{*} \omega_{j}\right) \bar{\psi}[f] .
$$

For $j \neq g$, the meromorphic forms $\pi^{*} \omega_{j}$ have only poles at $Q$. Hence

$$
\wedge e\left(\omega_{j}\right) \bar{\psi}[f]=0, \quad j \neq g .
$$

On the other hand, since $\pi^{*} \omega_{g}$ has a pole of order one at $P_{+}$and $P_{-}$with residues -1 and 1, respectively, and is holomorphic outside the points $P_{ \pm}$and $Q$ and $f\left(P_{+}\right)=-1$, $f\left(P_{-}\right)=0$ we have that

$$
\operatorname{Res}_{Q}\left(f \pi^{*} \omega_{g}\right)=-\operatorname{Res}_{P_{+}}\left(f \pi^{*} \omega_{g}\right)=-1 .
$$

Thus we conclude that

$$
\begin{aligned}
\langle\widetilde{\Phi} \mid 0 \otimes 0 \otimes u\rangle & =\left\langle\omega(\mathfrak{X}) \mid \rho_{Q}(\bar{\psi}[f]) u\right\rangle=\left\langle\omega(\mathfrak{X}) \rho_{Q}(\bar{\psi}[f]) \mid u\right\rangle \\
& =(-1)^{g-1} \operatorname{Res}_{Q}\left(f \omega_{g}\right)\left\langle\cdots \wedge e\left(\omega_{g+1}\right) \wedge e\left(\omega_{g-1}\right) \wedge \cdots \wedge e\left(\omega_{1}\right) \mid u\right\rangle \\
& =(-1)^{g}\langle\omega(\widehat{\mathfrak{X}},\{\widehat{\alpha}, \widehat{\beta}\}) \mid u\rangle .
\end{aligned}
$$

QED

For an $N$-pointed curve $\mathfrak{X}=\left(C ; Q_{1}, \ldots, Q_{N} ; \xi_{1}, \ldots, \xi_{N}\right)$ we define the preferred element $\langle\omega(\mathfrak{X},\{\alpha, \beta\})|$ by

$$
\langle\omega(\mathfrak{X},\{\alpha, \beta\})|=\iota^{*-1}\left\langle\omega\left(\mathfrak{X}_{1},\{\alpha, \beta\}\right)\right|
$$


where $\mathfrak{X}_{1}=\left(C ; Q_{1}, \xi_{1}\right)$ and $\iota^{*}: \mathcal{V}^{\dagger}{ }_{\mathrm{ab}}(\mathfrak{X}) \rightarrow \mathcal{V}^{\dagger}{ }_{\mathrm{ab}}\left(\mathfrak{X}_{1}\right)$ is defined by applying Theorem 3.4 several times. Note by Theorem 6.4 that if we choose to use the pair $\left(Q_{j}, \xi_{j}\right)$ instead of $\left(Q_{1}, \xi_{1}\right)$ in defining $\mathfrak{X}_{1}$, we get the same preferred element.

For an $N$-pointed nodal curve $\mathfrak{X}=\left(C ; Q_{1}, \ldots, Q_{N} ; \xi_{1}, \ldots, \xi_{N}\right)$ with formal coordinates such that the normalization $\widetilde{C}$ of $C$ is an irreducible curve, we can define the preferred element by generalizing the discussion just before Theorem 6.5 in the obvious way.

We can however not apply the same method to define the preferred element for onepointed nodal curves $(C ; Q, \xi)$ whose normalization $\widetilde{C}$ is disconnected. This is simply because a holomorphic one-form with support on one component will have zero Taylor expansion at all points of the curve not contained in that component.

Therefore, we need to find another definition. For simplicity, in the following, we shall consider a two-pointed nodal curve $\mathfrak{X}=\left(C ; Q_{1}, Q_{2} ; \xi_{1}, \xi_{2}\right)$ with formal coordinates such that the normalized curve $\widetilde{C}$ has two connected components $C_{1}$ and $C_{2}$. Assume that $Q_{1}, P_{+} \in C_{1}$ and $Q_{2}, P_{-} \in C_{2}$. Put $\mathfrak{X}_{1}=\left(C_{1} ; Q_{1}, \xi_{1}\right), \mathfrak{X}_{2}=\left(C_{2} ; Q_{2}, \xi_{2}\right) \widetilde{\mathfrak{X}}_{1}=$ $\left(C_{1} ; Q_{1}, P_{+}, \xi_{1}, z\right), \widetilde{\mathfrak{X}}_{2}=\left(C_{2} ; Q_{2}, P_{-}, \xi_{2}, w\right), \widetilde{\mathfrak{X}}=\left(C_{1} \cup C_{2} ; Q_{1}, Q_{2}, P_{+}, P_{-}, \xi_{1}, \xi_{2}, z, w\right)$. Then by Theorem 3.4 and Theorem 3.5 we have isomorphisms

$$
\begin{aligned}
\iota_{j}^{*}: \mathcal{V}^{\dagger}{ }_{\mathrm{ab}}\left(\widetilde{\mathfrak{X}}_{j}\right) & \cong \mathcal{V}_{\mathrm{ab}}^{\dagger}\left(\mathfrak{X}_{j}\right), \quad j=1,2 \\
\iota_{+,-}^{*}: \mathcal{V}_{\mathrm{ab}}^{\dagger}(\widetilde{\mathfrak{X}}) & \cong \mathcal{V}_{\mathrm{ab}}^{\dagger}(\mathfrak{X}) .
\end{aligned}
$$

Moreover, by Proposition 3.1 we have

$$
\mathcal{V}^{\dagger}{ }_{\mathrm{ab}}(\widetilde{\mathfrak{X}})=\mathcal{V}_{\mathrm{ab}}^{\dagger}\left(\widetilde{\mathfrak{X}}_{1}\right) \otimes \mathcal{V}^{\dagger}{ }_{\mathrm{ab}}\left(\widetilde{\mathfrak{X}}_{2}\right) .
$$

Choose symplectic bases $\left\{\alpha^{(i)}, \beta^{(i)}\right\}$ of $H_{1}\left(C_{i}, \mathbf{Z}\right)$. Then, $\{\alpha, \beta\}=\left\{\alpha^{(1)}, \alpha^{(2)}, \beta^{(1)}, \beta^{(2)}\right\}$ is a symplectic basis of $H_{1}(C, \mathbf{Z})$. Put

$$
\begin{aligned}
\left\langle\Phi_{1}\right| & =\iota_{1}^{*-1}\left(\left\langle\omega\left(\mathfrak{X}_{1},\left\{\alpha^{(1)}, \beta^{(1)}\right\}\right)\right|\right), \\
\left\langle\Phi_{2}\right| & =\iota_{2}^{*-1}\left(\left\langle\omega\left(\mathfrak{X}_{2},\left\{\alpha^{(2)}, \beta^{(2)}\right\}\right)\right|\right), \\
\langle\Phi| & =\left\langle\Phi_{1}\right| \otimes\left\langle\Phi_{2}\right| .
\end{aligned}
$$

Finally define

$$
\langle\omega(\mathfrak{X},\{\alpha, \beta\})|=\iota_{+,-}^{*}(\langle\Phi|) .
$$

For a general $N$-pointed nodal curve $\mathfrak{X}=\left(C ; Q_{1}, \ldots, Q_{N} ; \xi_{1}, \ldots, \xi_{N}\right)$ we define the preferred element $\langle\omega(\mathfrak{X},\{\alpha, \beta\})|$ in a similar way. The following lemma plays an important role in characterizing the preferred section.

Lemma 6.3. Under the same notation as above, let the genera of $C_{1}$ and $C_{2}$ be $g_{1}$ and $g_{2}$, respectively. Let $1=m_{1}<m_{2}<\cdots<m_{g_{1}} \leq 2 g_{1}-1$ and $1<n_{1}<n_{2}<\cdots<n_{g_{2}} \leq$ $2 g_{2}-1$ be the Weierstrass gap values of $C_{1}$ at $Q_{1}$ and $C_{2}$ at $Q_{2}$, respectively. Put

$$
\left|u_{1}\right\rangle=\bar{e}_{m_{g_{1}}-1 / 2} \wedge \cdots \wedge \bar{e}_{m_{1}-1 / 2} \wedge|-1\rangle, \quad\left|u_{2}\right\rangle=\bar{e}_{n_{g_{2}}-1 / 2} \wedge \cdots \wedge \bar{e}_{n_{1}-1 / 2} \wedge|0\rangle .
$$

Then we have that

$$
\left\langle\left(\iota_{+,-}^{*}\right)^{-1} \omega(\mathfrak{X},\{\alpha, \beta\}) \mid u_{1} \otimes u_{2}\right\rangle=\left|\begin{array}{ccc}
I_{m_{g_{1}}}^{1}\left(Q_{1}\right) & \cdots & I_{m_{g_{1}}}^{g_{1}}\left(Q_{1}\right) \\
\vdots & \ddots & \vdots \\
I_{m_{1}}^{1}\left(Q_{1}\right) & \cdots & I_{m_{1}}^{g_{1}}\left(Q_{1}\right)
\end{array}\right| \cdot\left|\begin{array}{ccc}
I_{n_{g_{2}}}^{g_{1}+1}\left(Q_{2}\right) & \cdots & I_{n_{g_{2}}}^{g_{1}+g_{2}}\left(Q_{2}\right) \\
\vdots & \ddots & \vdots \\
I_{n_{1}}^{g_{1}+1}\left(Q_{2}\right) & \cdots & I_{n_{1}}^{g_{1}+g_{2}}\left(Q_{2}\right)
\end{array}\right| \neq 0
$$


where $\left\{\omega_{1}, \ldots, \omega_{g_{1}}\right\}$ and $\left\{\omega_{g_{1}+1}, \ldots, \omega_{g_{1}+g_{2}}\right\}$ are the normalized bases of holomorphic oneforms on $C_{1}$ and $C_{2}$, respectively, and

$$
\left(\sum_{m=1}^{\infty} I_{m}^{k}\left(Q_{1}\right) \xi_{1}^{m-1}\right) d \xi_{1}, \quad 1 \leq k \leq g_{1}
$$

is the Taylor expansion of $\omega_{k}$ at $Q_{1}$ and

$$
\left(\sum_{n=1}^{\infty} I_{n}^{g_{1}+i}\left(Q_{2}\right) \xi_{2}^{n-1}\right) d \xi_{2}, \quad 1 \leq i \leq g_{2} .
$$

is the Taylor expansion of $\omega_{g_{1}+i}$ at $Q_{2}$.

Proof. Let $\omega_{1}$ be a meromorphic one-form on $C_{1}$ which is holomorphic outside $P_{+}$ and $Q_{1}$ such that at $P_{+}$and $Q_{1}, \omega_{1}$ has the Laurent expansions:

$$
\begin{aligned}
\omega_{1,+} & =\frac{d z}{z}+\text { holomorphic } \\
\omega_{1, Q_{1}} & =-\frac{d \xi_{1}}{\xi_{1}}+\text { holomorphic. }
\end{aligned}
$$

Similarly let $\omega_{2}$ be a meromorphic one-form on $C_{2}$ which is holomorphic outside $P_{-}$and $Q_{2}$ such that at $P_{-}$and $Q_{2} \omega_{2}$ has the Laurent expansions:

$$
\begin{aligned}
\omega_{2,-} & =-\frac{d w}{w}+\text { holomorphic } \\
\omega_{2, Q_{2}} & =\frac{d \xi_{2}}{\xi_{2}}+\text { holomorphic. }
\end{aligned}
$$

Then, we have that

$$
\psi\left[\omega_{1,+}\right]|0\rangle=|-1\rangle, \quad-\psi\left[\omega_{1,-}\right]|0\rangle=|-1\rangle .
$$

and therefore, we have that

$$
\begin{aligned}
\left\langle\left(\iota_{+,-}^{*}\right)^{-1} \omega(\mathfrak{X},\{\alpha, \beta\}) \mid u_{1} \otimes u_{2}\right\rangle= & \left\langle\Phi \mid 0_{+,-} \otimes u_{1} \otimes u_{2}\right\rangle \\
= & \left\langle\Phi \mid 0 \otimes-1 \otimes u_{1} \otimes u_{2}\right\rangle-\left\langle\Phi \mid-1 \otimes 0 \otimes u_{1} \otimes u_{2}\right\rangle \\
= & \left\langle\Phi_{1} \mid 0 \otimes u_{1}\right\rangle\left\langle\Phi_{2} \mid-1 \otimes u_{2}\right\rangle-\left\langle\Phi_{1} \mid-1 \otimes u_{1}\right\rangle\left\langle\Phi_{2} \mid 0 \otimes u_{2}\right\rangle \\
= & \left.\left\langle\omega\left(\mathfrak{X}_{1},\left\{\alpha^{(1)}, \beta^{(1)}\right\}\right) \mid u_{1}\right\rangle\left\langle\Phi_{2}\left|\psi\left[\omega_{1,+}\right]\right| 0\right\rangle \otimes u_{2}\right\rangle \\
& \left.+\left\langle\Phi_{1}\left|\psi\left[\omega_{1,-}\right]\right| 0\right\rangle \otimes u_{1}\right\rangle\left\langle\omega\left(\mathfrak{X}_{2},\left\{\alpha^{(2)}, \beta^{(2)}\right\}\right) \mid u_{2}\right\rangle \\
= & \left\langle\omega\left(\mathfrak{X}_{1},\left\{\alpha^{(1)}, \beta^{(1)}\right\}\right) \mid u_{1}\right\rangle\left\langle\omega\left(\mathfrak{X}_{2},\left\{\alpha^{(2)}, \beta^{(2)}\right\}\right) \mid \psi\left[\omega_{1, Q_{2}}\right] u_{2}\right\rangle \\
& +\left\langle\omega\left(\mathfrak{X}_{1},\left\{\alpha^{(1)}, \beta^{(1)}\right\}\right) \mid \psi\left[\omega_{1, Q_{1}}\right] u_{1}\right\rangle\left\langle\omega\left(\mathfrak{X}_{2},\left\{\alpha^{(2)}, \beta^{(2)}\right\}\right) \mid u_{2}\right\rangle
\end{aligned}
$$

Since

$$
\psi\left[\omega_{1, Q_{2}}\right]\left|u_{2}\right\rangle=\bar{e}_{n_{g_{2}-1 / 2}} \wedge \cdots \wedge \bar{e}_{n_{1}-1 / 2} \wedge|-1\rangle+* \wedge|0\rangle
$$

and $\left\langle\omega\left(\mathfrak{X}_{2},\left\{\alpha^{(2)}, \beta^{(2)}\right\}\right)\right|$ does not contains the term $e^{-1 / 2}$, we have that

$$
\begin{aligned}
\left\langle\omega\left(\mathfrak{X}_{2},\left\{\alpha^{(2)}, \beta^{(2)}\right\}\right) \mid \psi\left[\omega_{1, Q_{2}}\right] u_{2}\right\rangle & =\left\langle\omega\left(\mathfrak{X}_{2},\left\{\alpha^{(2)}, \beta^{(2)}\right\}\right)\left|\bar{e}_{n_{g_{2}}-1 / 2} \wedge \cdots \bar{e}_{n_{1}-1 / 2} \wedge\right|-1\right\rangle \\
& =\left|\begin{array}{ccc}
I_{n_{g_{2}}}^{g_{1}+1}\left(Q_{2}\right) & \cdots & I_{n_{g_{2}}}^{g_{1}+g_{2}}\left(Q_{2}\right) \\
\vdots & \ddots & \vdots \\
I_{n_{1}}^{g_{1}+1}\left(Q_{2}\right) & \cdots & I_{n_{1}}^{g_{1}+g_{2}}\left(Q_{2}\right)
\end{array}\right| \neq 0
\end{aligned}
$$


Since by the same reason $\left\langle\omega\left(\mathfrak{X}_{1},\left\{\alpha^{(1)}, \beta^{(1)}\right\}\right) \mid \psi\left[\omega_{1, Q_{1}}\right] u_{1}\right\rangle\left\langle\omega\left(\mathfrak{X}_{2},\left\{\alpha^{(2)}, \beta^{(2)}\right\}\right) \mid u_{2}\right\rangle=0$ and

$$
\left\langle\omega\left(\mathfrak{X}_{1},\left\{\alpha^{(1)}, \beta^{(1)}\right\}\right) \mid u_{1}\right\rangle=\left|\begin{array}{ccc}
I_{m_{g_{1}}}^{1}\left(Q_{1}\right) & \cdots & I_{m_{g_{1}}}^{g_{1}}\left(Q_{1}\right) \\
\vdots & \ddots & \vdots \\
I_{m_{1}}^{1}\left(Q_{1}\right) & \cdots & I_{m_{1}}^{g_{1}}\left(Q_{1}\right)
\end{array}\right| \neq 0
$$

we obtain the desired result.

Let $\mathfrak{F}=\left(\pi: \mathcal{C} \rightarrow \mathcal{B}, s_{1}, \ldots, s_{N}, \xi_{1}, \ldots, \xi_{N}\right)$ be a family of $N$-pointed smooth curves. For any point $b \in \mathcal{B}$ there exists an open neighbourhood $U_{b}$ such that $\pi^{-1}\left(U_{b}\right)$ is topologically trivial so that we can choose smoothly varying symplectic bases

$$
\left\{\alpha_{1}(t), \ldots, \alpha_{g}(t), \beta_{1}(t), \ldots, \beta_{g}(t)\right\}, \quad t \in \mathcal{B} .
$$

Then we can define $\left\langle\omega\left(\mathfrak{X}_{t},\{\alpha(t), \beta(t)\}\right)\right|$ where $\mathfrak{X}_{t}=\left(\pi^{-1}(t), s_{1}(t), \xi_{1}\right)$.

Theorem 6.6. The section $\left\langle\omega\left(\mathfrak{X}_{t},\{\alpha(t), \beta(t)\}\right)\right|$ is a holomorphic section of $\mathcal{V}^{\dagger}{ }_{\mathrm{ab}}(\mathfrak{F})$ over $U_{b}$.

Proof. Put $\mathfrak{F}^{\prime}=\left(\pi: \mathcal{C} \rightarrow \mathcal{B}, s_{1}, \xi_{1}\right)$. Then, by Theorem 3.4 it is easy to show that we get an isomorphism $\mathcal{V}^{\dagger} \mathrm{ab}(\mathfrak{F}) \cong \mathcal{V}^{\dagger}{ }_{\mathrm{ab}}\left(\mathfrak{F}^{\prime}\right)$ by the propagation of vacua construction. Hence it is enough to show that $\left\langle\omega\left(\mathfrak{X}_{t},\{\alpha(t), \beta(t)\}\right)\right|$ depends holomorphically on $t$. This follows, since $\omega_{s_{1}(t)}^{(n)}$ varies holomorphically in $t$.

QED

Finally, we analyze the preferred section for families of deformations of nodal curves.

Let $C_{0}$ be a complete curve with only one ordinary double point $P$ such that $C_{0} \backslash\{P\}$ is non-singular. Let $Q_{1}, Q_{2}, \ldots, Q_{N}$ be distinct non-singular points on $C_{0}$. Let $\nu: \widetilde{C}_{0} \rightarrow C_{0}$ be the normalization of the singular curve. Put $\left\{P_{+}, P_{-}\right\}=\nu^{-1}(P)$. Note that the normalization might or might not be connected but assume that each component contains at least one $Q_{j}$. Let $\mathfrak{F}=\left(\pi: \mathcal{C} \rightarrow D ; \sigma_{1}, \ldots, \sigma_{N} ; \xi_{1}, \ldots, \xi_{N}\right)$ be the family constructed from $\left(C_{0}, Q_{1}, \ldots, Q_{N}, \xi_{1}, \ldots \xi_{N}\right)$ as described right before Lemma 5.7. Suppose we now have a continuous basis $\left\{\alpha_{i}(t), \beta_{i}(t)\right\}$ of $H_{1}\left(\pi^{-1}(t), \mathbf{Z}\right), t \in(0,1) \subset D$, such that we get a well defined limit as $t$ goes to zero, which gives a symplectic basis, say $\left\{\alpha_{1}(0), \ldots, \alpha_{g-1}(0), \alpha_{g}(0), \beta_{1}(0), \ldots, \beta_{g-1}(0)\right\}$ of $H_{1}\left(C_{0}, \mathbf{Z}\right)$ as described above for nodal curves and $\beta_{g}(0)=0$. Let $\mathfrak{X}_{t}=\left(\pi^{-1}(t), s_{1}(t), \ldots s_{N}(t), \xi_{1}, \ldots \xi_{N}\right)$.

Theorem 6.7. We have that

$$
\left\langle\omega\left(\mathfrak{X}_{0},\{\alpha(0), \beta(0)\}\right)\right|=\lim _{t \rightarrow 0}\left\langle\omega\left(\mathfrak{X}_{t},\{\alpha(t), \beta(t)\}\right)\right| .
$$

Proof. Assume the curve $\widetilde{C}_{0}$ is connected. It is then enough to prove the theorem when $N=1$. Note by [F, Proposition 3.7] that if $\left\{\omega_{1}, \ldots, \omega_{g}\right\}$ is a normalized basis of holomorphic one-forms on $C_{0}$, then a normalized bases of holomorphic one-forms for the family is of the form:

$$
\begin{aligned}
\omega_{i}(x, t) & =\omega_{i}(x)+\frac{1}{4} t\left(\omega_{i}\left(P_{+}\right)-\omega_{i}\left(P_{-}\right)\right)\left(\omega\left(x, P_{+}\right)-\omega\left(x, P_{-}\right)\right)+O\left(t^{2}\right), \quad 1 \leq i \leq g-1 \\
\omega_{g}(x, t) & =\omega_{P_{+}-P_{-}}(x)+t u_{g}(x)+O\left(t^{2}\right) .
\end{aligned}
$$


Here $\left\{\omega_{1}(x), \ldots, \omega_{g-1}(x)\right\}$ is a normalized basis of holomorphic one-forms of $\widetilde{C}_{0}$, and $\omega_{i}\left(P_{+}\right)$is the number $f_{i}(0)$ where in a neighbourhood of $P_{+}, \omega_{i}$ is expressed as $f_{i}(z) d z$. The number $\omega_{i}\left(P_{-}\right)$is defined similarly. Moreover, $\omega(x, y)$ is the normalized bidifferential of the curve $\widetilde{C}_{0}$ and in a neighbourhood of $\left(x, P_{+}\right)$if we express $\omega(x, y)=f(x, z) d x d z$ then $\omega\left(x, P_{+}\right)$is defined as $f(x, 0) d x$. The one-form $\omega\left(x, P_{-}\right)$defined similarly. The form $\omega_{P_{+}-P_{-}}(x)$ is a meromorphic one-form of $\widetilde{C}_{0}$ which has a pole of order one at $P_{+}$with residue 1 , pole of order one at $P_{-}$with residue -1 and holomorphic outside $P_{+}$and $P_{-}$, and $u_{g}$ is a meromorphic one-form on $\widetilde{C}_{0}$ which has only poles at $P_{ \pm}$of order three. Finally, the expression $O\left(t^{2}\right)$ means that it is a holomorphic one-form on $\widetilde{C}_{0} \backslash\left\{P_{+}, P_{-}\right\}$ and $\lim _{t \rightarrow 0} \frac{O\left(t^{2}\right)}{t^{2}}$ is a holomorphic one-form on $\widetilde{C}_{0} \backslash\left\{P_{+}, P_{-}\right\}$.

Let

$$
\omega_{i}(x, t)=\left(\sum_{n=1}^{\infty} I_{n}^{i}(t) \xi^{n-1}\right) d \xi
$$

for $i=1, \ldots g$.

The meromorphic one-forms $\omega_{g+n}(t)$ on $C_{t}$, as defined by (6.1) and (6.2) has a pole only at $Q_{1}=s_{1}(t)$ of order $n+1$ with Laurent expansion at $s_{1}(t)$ of the form

$$
\frac{d \xi_{1}}{\xi_{1}^{n+1}}+\text { holomorphic }
$$

and satisfies

$$
\int_{\alpha_{i}(t)} \omega_{g+n}(t)=-\frac{2 \pi \sqrt{-1} I_{n}^{i}(t)}{n}, \int_{\beta_{i}(t)} \omega_{g+n}(t)=0, \quad 1 \leq i \leq g, \quad n \geq 0 .
$$

Then,

$$
\left\langle\omega\left(\mathfrak{X}_{t},\{\alpha(t), \beta(t)\}\right)\right|=\left\langle\cdots \wedge e\left(\omega_{m}(t)\right) \wedge \cdots \wedge e\left(\omega_{2}(t)\right) \wedge e\left(\omega_{1}(t)\right)\right| .
$$

Thus we conclude that

$$
\lim _{t \rightarrow 0}\left\langle\omega\left(\mathfrak{X}_{t},\{\alpha(t), \beta(t)\}\right)\right|=\left\langle\omega\left(\mathfrak{X}_{0},\{\alpha(0), \beta(0)\}\right)\right| .
$$

Next, assume that the curve $\widetilde{C}_{0}$ has two connected components $C_{1}$ and $C_{2}$, and that $Q_{1}, P_{+} \in C_{1}$ and $Q_{2}, P_{-} \in C_{2}$. Moreover, it is enough to consider the case $N=2$. Let $\left\{\omega_{1}, \ldots, \omega_{g_{1}}\right\}$ and $\left\{\omega_{g_{1}+1}, \ldots, \omega_{g_{1}+g_{2}}\right\}$ be normalized bases of holomorphic one-forms of $C_{1}$ and $C_{2}$, respectively. Then we can find a family $\left\{\omega_{1}(t), \ldots, \omega_{g_{1}+g_{2}}(t)\right\}$ of normalized bases of the family $\pi: \mathcal{C}^{*}=\pi^{-1}(D \backslash\{0\}) \rightarrow D \backslash\{0\}$ such that $\lim _{t \rightarrow 0} \omega_{k}(t)=\omega_{k}$ according to Proposition $3.1[\mathrm{~F}]$. Here we regard $\omega_{k}$ as a holomorphic section of the dualizing sheaf $\omega_{C_{0}}$ of the nodal curve, by extending it by zero to the other component.

Over $D \backslash\{0\}\left\langle\omega\left(\mathfrak{X}_{t},\{\alpha(t), \beta(t)\}\right)\right|$ is a holomorphic section of the sheaf of ghost vacua. Let $1=m_{1}<m_{2}<\cdots<m_{g_{1}} \leq 2 g_{1}-1$ and $1=n_{1}<n_{2}<\cdots<n_{g_{2}} \leq 2 g_{2}-1$ be the Weierstrass gap values of $C_{1}$ at $Q_{1}$ and $C_{2}$ at $Q_{2}$, respectively. Put

$$
\left|u_{1}\right\rangle=\bar{e}_{m_{g_{1}}-1 / 2} \wedge \cdots \wedge \bar{e}_{m_{1}-1 / 2} \wedge|-1\rangle, \quad\left|u_{2}\right\rangle=\bar{e}_{n_{g_{2}}-1 / 2} \wedge \cdots \wedge \bar{e}_{n_{1}-1 / 2} \wedge|0\rangle .
$$

Choose sections $f_{j}$ of $\pi_{*} \mathcal{O}_{\mathcal{C}}\left(n_{j} s_{2}(D)+* s_{1}(D)\right)$ over $D$ such that it has a Laurent expansion

$$
f_{j, 2}=\frac{1}{\xi_{2}^{n_{j}}}+\text { holomorphic }
$$


along $s_{2}(D)$. Put also

$$
\mathfrak{X}_{t}^{\prime}=\left(\pi^{-1}(t) ; s_{1}(t) ; \xi_{1}\right) \text {. }
$$

Then we have that

$$
\begin{aligned}
\left\langle\omega\left(\mathfrak{X}_{t},\{\alpha(t), \beta(t)\}\right) \mid u_{1} \otimes u_{2}\right\rangle & =\left\langle\omega\left(\mathfrak{X}_{t},\{\alpha(t), \beta(t)\}\right)\left|u_{1} \otimes \bar{\psi}\left[f_{g_{2}, 2}\right] \bar{\psi}\left[f_{g_{2}-1,2}\right] \cdots \bar{\psi}\left[f_{1,2}\right]\right| 0\right\rangle \\
& =(-1)^{g_{2}-1}\left\langle\omega\left(\mathfrak{X}_{t},\{\alpha(t), \beta(t)\}\right)\left|\bar{\psi}\left[f_{1,2}\right] \bar{\psi}\left[f_{2,2}\right] \cdots \bar{\psi}\left[f_{g_{2}, 2}\right]\right| u_{1} \otimes 0\right\rangle \\
& =(-1)^{g_{2}-1}\left\langle\omega\left(\mathfrak{X}_{t}^{\prime},\{\alpha(t), \beta(t)\}\right)\left|\bar{\psi}\left[f_{1,2}\right] \bar{\psi}\left[f_{2,2}\right] \cdots \bar{\psi}\left[f_{g_{2}, 2}\right]\right| u_{1}\right\rangle \\
& =(-1)^{g_{2}-1}\left|\begin{array}{ccc}
\operatorname{Res}_{Q_{1}}\left(f_{1} \omega_{1}(t)\right) & \cdots & \operatorname{Res}_{Q_{1}}\left(f_{1} \omega_{g_{1}+g_{2}}(t)\right) \\
\vdots & \ddots & \vdots \\
\operatorname{Res}_{Q_{1}}\left(f_{g_{2}} \omega_{1}(t)\right) & \cdots & \operatorname{Res}_{Q_{1}}\left(f_{g_{2}} \omega_{g_{1}+g_{2}}(t)\right) \\
I_{m_{1}}^{1}\left(Q_{1}, t\right) & \cdots & I_{m_{1}}^{g_{1}+g_{2}}\left(Q_{1}, t\right) \\
\vdots & \ddots & \vdots \\
I_{m_{g_{1}}}^{1}\left(Q_{1}, t\right) & \cdots & I_{m_{g_{1}}}^{g_{1}+g_{2}}\left(Q_{1}, t\right)
\end{array}\right|
\end{aligned}
$$

where the Taylor expansion of $\omega_{k}(t)$ along $s_{1}(D)$ and $s_{2}(D)$ are written as

$$
\begin{aligned}
& \omega_{k}(t)=\sum_{m=1}^{\infty} I_{m}^{k}\left(Q_{1}, t\right) \xi_{1}^{m-1} \\
& \omega_{k}(t)=\sum_{n=1}^{\infty} I_{n}^{k}\left(Q_{2}, t\right) \xi_{1}^{n-1}
\end{aligned}
$$

Now by the residue theorem we have

$$
\operatorname{Res}_{Q_{1}}\left(f_{j} \omega_{k}(t)\right)=-\operatorname{Res}_{Q_{2}}\left(f_{j} \omega_{k}(t)\right)=-I_{n_{j}}^{k}\left(Q_{2}, t\right) .
$$

Since for any positive integers $m, n$ we have

$$
\begin{aligned}
\lim _{t \rightarrow 0} I_{m}^{k}\left(Q_{1}, t\right) & =I_{m}^{k}\left(Q_{1}\right), \quad 1 \leq k \leq g_{1}, \\
\lim _{t \rightarrow 0} I_{n}^{g_{1}+i}\left(Q_{1}, t\right) & =0, \quad 1 \leq i \leq g_{2}, \\
\lim _{t \rightarrow 0} I_{m}^{k}\left(Q_{2}, t\right) & =0, \quad 1 \leq k \leq g_{1}, \\
\lim _{t \rightarrow 0} I_{n}^{g_{1}+i}\left(Q_{2}, t\right) & =I_{n}^{g_{1}+i}\left(Q_{2}\right), 1 \leq i \leq g_{2},
\end{aligned}
$$


we conclude that

$$
\begin{aligned}
& \lim _{t \rightarrow 0}\left\langle\omega\left(\mathfrak{X}_{t},\{\alpha(t), \beta(t)\}\right) \mid u_{1} \otimes u_{2}\right\rangle \\
& =(-1)^{g_{2}-1} \lim _{t \rightarrow 0}\left|\begin{array}{ccc}
-I_{n_{1}}^{1}\left(Q_{2}, t\right) & \cdots & -I_{n_{1}}^{g_{1}+g_{2}}\left(Q_{2}, t\right) \\
\vdots & \ddots & \vdots \\
-I_{n_{g_{2}}}^{1}\left(Q_{2}, t\right) & \cdots & -I_{n_{g_{2}}}^{g_{1}+g_{2}}\left(Q_{2}, t\right) \\
I_{m_{1}}^{1}\left(Q_{1}, t\right) & \cdots & I_{m_{1}}^{g_{1}+g_{2}}\left(Q_{1}, t\right) \\
\vdots & \ddots & \vdots \\
I_{m_{g_{1}}}^{1}\left(Q_{1}, t\right) & \cdots & I_{m_{g_{1}}}^{g_{1}+g_{2}}\left(Q_{1}, t\right)
\end{array}\right| \\
& =-\left|\begin{array}{ccc}
I_{m_{1}}^{1}\left(Q_{1}\right) & \cdots & I_{m_{1}}^{g_{1}}\left(Q_{1}\right) \\
\vdots & \ddots & \vdots \\
I_{m_{g_{1}}}^{1}\left(Q_{1}\right) & \cdots & I_{m_{g_{1}}}^{g_{1}}\left(Q_{1}\right)
\end{array}\right| \cdot\left|\begin{array}{ccc}
I_{n_{1}}^{g_{1}+1}\left(Q_{2}\right) & \cdots & I_{n_{1}}^{g_{1}+g_{2}}\left(Q_{2}\right) \\
\vdots & \ddots & \vdots \\
I_{n_{g_{2}}}^{g_{1}+1}\left(Q_{2}\right) & \cdots & I_{n_{g_{2}}}^{g_{1}+g_{2}}\left(Q_{2}\right)
\end{array}\right| \\
& =-\left\langle\omega\left(\mathfrak{X}_{0},\{\alpha(0), \beta(0)\}\right) \mid u_{1} \otimes u_{2}\right\rangle \neq 0 .
\end{aligned}
$$

Hence if $\lim _{t \rightarrow 0}\left\langle\omega\left(\mathfrak{X}_{t},\{\alpha(t), \beta(t)\}\right)\right|$ exists, then it is $-\left\langle\omega\left(\mathfrak{X}_{0},\{\alpha(0), \beta(0)\}\right)\right|$.

Now let us show that $\lim _{t \rightarrow 0}\left\langle\omega\left(\mathfrak{X}_{t},\{\alpha(t), \beta(t)\}\right)\right|$ exists. Note that for $t \neq 0,\left\langle\omega\left(\mathfrak{X}_{t}^{\prime},\{\alpha(t), \beta(t)\}\right)\right|$ is given by

$$
\left\langle\cdots \wedge e\left(\omega_{m}(t)\right) \wedge \cdots \wedge e\left(\omega_{2}(t)\right) \wedge e\left(\omega_{1}(t)\right)\right|
$$

where $\left\{\omega_{1}(0), \ldots, \omega_{g}(0)\right\}$ is a normalized basis of holomorphic one-forms of $C_{0}$ and $\omega_{g+i}(0)$ is a normalized meromorphic one-form which has only a pole at $s_{1}(0)=Q_{1}$. Now for $t \neq 0\left\{\omega_{1}(0), \ldots, \omega_{g}(0)\right\}$ is defined by using Theorem 3.4. This means that for $\left|v_{1} \otimes v_{2}\right\rangle \in \mathcal{F}_{2}$, the evaluation $\left\langle\omega\left(\mathfrak{X}_{t},\{\alpha(t), \beta(t)\}\right) \mid v_{1} \otimes v_{2}\right\rangle$ is reduced to the evaluation of $\left\langle\omega\left(\mathfrak{X}_{t}^{\prime},\{\alpha(t), \beta(t)\}\right) \mid v_{1}^{\prime}\right\rangle$ so that we can use similar arguments as above. For example let us calculate $\left\langle\omega\left(\mathfrak{X}_{t},\{\alpha(t), \beta(t)\}\right) \mid v_{1} \otimes v_{2}\right\rangle$ for

$$
\begin{aligned}
& v_{1}=\bar{e}_{k_{1}+1 / 2} \wedge|0\rangle, \quad 1 \leq k_{1}, \\
& v_{2}=\bar{e}_{l_{g}-1 / 2} \wedge \cdots \wedge \bar{e}_{l_{g}-1 / 2} \wedge i\left(\bar{e}_{-m-1 / 2}\right)|-1\rangle, \quad 0 \leq l_{1}<\cdots<l_{g}, \quad 0 \leq m .
\end{aligned}
$$

Choose meromorphic functions $f_{i} \in H^{0}\left(D, \pi_{*} \mathcal{O}_{\mathcal{C}}\left(*\left(s_{1}(D)+s_{2}(D)\right)\right)\right.$ which have the Laurent expansion along $s_{2}(D)$ :

$$
f_{i, 2}=\frac{1}{\xi_{2}^{l_{i}}}+\text { holomorphic. }
$$

Also choose $\tau \in H^{0}\left(D, \pi_{*} \omega_{\mathcal{C}}\left(*\left(s_{1}(D)+s_{2}(D)\right)\right)\right.$ which has the Laurent expansion along $s_{2}(D)$ :

$$
\tau_{2}=\left(\frac{1}{\xi_{2}^{m}}+\text { holomorphic }\right) d \xi_{2}
$$

Then by a similar argument as above

$$
\left\langle\omega\left(\mathfrak{X}_{t},\{\alpha(t), \beta(t)\}\right) \mid v_{1} \otimes v_{2}\right\rangle= \pm\left\langle\omega\left(\mathfrak{X}_{t}^{\prime},\{\alpha(t), \beta(t)\}\right)\left|\psi\left[\tau_{1}\right] \bar{\psi}\left[f_{1,1}\right] \cdots \bar{\psi}\left[f_{g, 1}\right]\right|-1\right\rangle .
$$

By a simple calculation we have that

$$
\begin{aligned}
\psi\left[\tau_{1}\right] \bar{\psi}\left[f_{1,1}\right] \cdots \bar{\psi}\left[f_{g, 1}\right]|-1\rangle= & \left.\sum_{i=1}^{g}(-1)^{i-1} \operatorname{Res}_{s_{1}(t)}\left(f_{i} \tau\right) \bar{\psi}\left[f_{1,1}\right] \cdots \bar{\psi}\left[f_{i-1,1}\right]\left|\bar{\psi}\left[f_{i+1,1}\right] \cdots\right|-1\right\rangle \\
& \bar{\psi}\left[f_{1,1}\right] \cdots \bar{\psi}\left[f_{g, 1}\right] \psi\left[\tau_{1}\right]|-1\rangle .
\end{aligned}
$$


Moreover, we have that

$$
\psi\left[\tau_{1}\right]|-1\rangle=\sum_{n=1}^{s} a_{n} \bar{e}_{-3 / 2} \wedge \cdots \wedge \bar{e}_{-n-1 / 2} \wedge \bar{e}_{-n_{3} / 2} \wedge \cdots
$$

Then, as above

$$
\begin{aligned}
& \left\langle\omega\left(\mathfrak{X}_{t}^{\prime},\{\alpha(t), \beta(t)\}\right)\left|\psi\left[\tau_{1}\right] \bar{\psi}\left[f_{1,1}\right] \cdots \bar{\psi}\left[f_{g, 1}\right]\right|-1\right\rangle \\
& = \pm \sum_{i=1}^{g}(-1)^{i-1} \operatorname{Res}_{s_{1}(t)}\left(f_{i} \tau\right)\left|\begin{array}{ccc}
\operatorname{Res}_{s_{1}(t)}\left(f_{1} \omega_{1}(t)\right) \\
\vdots & \cdots & \operatorname{Res}_{s_{1}(t)}\left(f_{1} \omega_{g}(t)\right) \\
\operatorname{Res}_{s_{1}(t)}\left(f_{g} \omega_{1}(t)\right) & \cdots & \operatorname{Res}_{s_{1}(t)}\left(f_{g} \omega_{g}(t)\right)
\end{array}\right| \\
& +\sum_{n=1}^{s}\left(\left|\begin{array}{cccc}
\operatorname{Res}_{s_{1}(t)}\left(f_{1} \omega_{g+n}(t)\right) & \operatorname{Res}_{s_{1}(t)}\left(f_{1} \omega_{2}(t)\right) & \cdots & \operatorname{Res}_{s_{1}(t)}\left(f_{1} \omega_{g}(t)\right) \\
\vdots & \ddots & \vdots & \\
\operatorname{Res}_{s_{1}(t)}\left(f_{g} \omega_{g+n}(t)\right) & \operatorname{Res}_{s_{1}(t)}\left(f_{g} \omega_{2}(t)\right) & \cdots & \operatorname{Res}_{s_{1}(t)}\left(f_{g} \omega_{g}(t)\right)
\end{array}\right|\right. \\
& +\left|\begin{array}{cccc}
\operatorname{Res}_{s_{1}(t)}\left(f_{1} \omega_{1}(t)\right) & \operatorname{Res}_{s_{1}(t)}\left(f_{1} \omega_{g+n}(t)\right) & \cdots & \operatorname{Res}_{s_{1}(t)}\left(f_{1} \omega_{g}(t)\right) \\
\vdots & \ddots & \vdots & \\
\operatorname{Res}_{s_{1}(t)}\left(f_{g} \omega_{1}(t)\right) & \operatorname{Res}_{s_{1}(t)}\left(f_{g} \omega_{g+n}(t)\right) & \cdots & \operatorname{Res}_{s_{1}(t)}\left(f_{g} \omega_{g}(t)\right)
\end{array}\right| \\
& \left.+\left|\begin{array}{cccc}
\operatorname{Res}_{s_{1}(t)}\left(f_{1} \omega_{1}(t)\right) & \operatorname{Res}_{s_{1}(t)}\left(f_{1} \omega_{2}(t)\right) & \cdots & \operatorname{Res}_{s_{1}(t)}\left(f_{1} \omega_{g+n}(t)\right) \\
\vdots & \ddots & \vdots & \\
\operatorname{Res}_{s_{1}(t)}\left(f_{g} \omega_{1}(t)\right) & \operatorname{Res}_{s_{1}(t)}\left(f_{g} \omega_{2}(t)\right) & \cdots & \operatorname{Res}_{s_{1}(t)}\left(f_{g} \omega_{g+n}(t)\right)
\end{array}\right|\right)
\end{aligned}
$$

which can be rewritten in the following form by using $\operatorname{Res}_{s_{1}(t)}\left(f_{i} \omega_{k}\right)=-\operatorname{Res}_{s_{2}(t)}\left(f_{i} \omega_{k}\right)$.

$$
\begin{gathered}
= \pm \sum_{i=1}^{g}(-1)^{i-1} \operatorname{Res}_{s_{1}(t)}\left(f_{i} \tau\right)\left|\begin{array}{ccc}
-\operatorname{Res}_{s_{2}(t)}\left(f_{1} \omega_{1}(t)\right) & \cdots & -\operatorname{Res}_{s_{2}(t)}\left(f_{1} \omega_{g}(t)\right) \\
\vdots & \ddots & \vdots \\
-\operatorname{Res}_{s_{2}(t)}\left(f_{g_{1}} \omega_{1}(t)\right) & \cdots & -\operatorname{Res}_{s_{2}(t)}\left(f_{g_{1}} \omega_{g}(t)\right) \\
\operatorname{Res}_{s_{1}(t)}\left(f_{g} \omega_{g_{1}+1}(t)\right) & \cdots & \operatorname{Res}_{s_{1}(t)}\left(f_{g} \omega_{g_{1}+1}(t)\right) \\
\vdots & \ddots & \vdots \\
\operatorname{Res}_{s_{1}(t)}\left(f_{g} \omega_{1}(t)\right) & \cdots & \operatorname{Res}_{s_{1}(t)}\left(f_{g} \omega_{g}(t)\right)
\end{array}\right| \\
+\sum_{n=1}^{s}\left(\begin{array}{cccc}
-\operatorname{Res}_{s_{2}(t)}\left(f_{1} \omega_{g+n}(t)\right) & -\operatorname{Res}_{s_{2}(t)}\left(f_{1} \omega_{2}(t)\right) & \cdots & -\operatorname{Res}_{s_{2}(t)}\left(f_{1} \omega_{g}(t)\right) \\
\vdots & \ddots & \vdots & \\
-\operatorname{Res}_{s_{2}(t)}\left(f_{g_{1}} \omega_{g+n}(t)\right) & -\operatorname{Res}_{s_{2}(t)}\left(f_{g_{1}} \omega_{2}(t)\right) & \cdots & -\operatorname{Res}_{s_{2}(t)}\left(f_{g_{1}} \omega_{g}(t)\right) \\
\operatorname{Res}_{s_{1}(t)}\left(f_{g_{1}+1} \omega_{g+n}(t)\right) & \operatorname{Res}_{s_{1}(t)}\left(f_{g_{1}+1} \omega_{2}(t)\right) & \cdots & \operatorname{Res}_{s_{1}(t)}\left(f_{g_{1}+1} \omega_{g}(t)\right) \\
\vdots & \ddots & \vdots & \\
\operatorname{Res}_{s_{1}(t)}\left(f_{g} \omega_{g+n}(t)\right) & \operatorname{Res}_{s_{1}(t)}\left(f_{g} \omega_{2}(t)\right) & \cdots & \operatorname{Res}_{s_{1}(t)}\left(f_{g} \omega_{g}(t)\right)
\end{array} \mid\right.
\end{gathered}
$$




$$
\begin{aligned}
& +\left|\begin{array}{cccc}
-\operatorname{Res}_{s_{2}(t)}\left(f_{1} \omega_{1}(t)\right) & -\operatorname{Res}_{s_{2}(t)}\left(f_{1} \omega_{g+n}(t)\right) & \cdots & -\operatorname{Res}_{s_{2}(t)}\left(f_{1} \omega_{g}(t)\right) \\
\vdots & \ddots & \vdots & \\
-\operatorname{Res}_{s_{2}(t)}\left(f_{g_{1}} \omega_{1}(t)\right) & -\operatorname{Res}_{s_{2}(t)}\left(f_{g_{1}} \omega_{g+n}(t)\right) & \cdots & -\operatorname{Res}_{s_{2}(t)}\left(f_{g_{1}} \omega_{g}(t)\right) \\
\operatorname{Res}_{s_{1}(t)}\left(f_{g_{1}+1} \omega_{1}(t)\right) & \operatorname{Res}_{s_{1}(t)}\left(f_{g_{1}+1} \omega_{g+n}(t)\right) & \cdots & \operatorname{Res}_{s_{1}(t)}\left(f_{g_{1}+1} \omega_{g}(t)\right) \\
\vdots & \ddots & \vdots & \\
\operatorname{Res}_{s_{1}(t)}\left(f_{g} \omega_{1}(t)\right) & \operatorname{Res}_{s_{1}(t)}\left(f_{g} \omega_{g+n}(t)\right) & \cdots & \operatorname{Res}_{s_{1}(t)}\left(f_{g} \omega_{g}(t)\right)
\end{array}\right| \\
& \left.+\left|\begin{array}{cccc}
\operatorname{Res}_{s_{1}(t)}\left(f_{1} \omega_{1}(t)\right) & \operatorname{Res}_{s_{1}(t)}\left(f_{1} \omega_{2}(t)\right) & \ldots & \operatorname{Res}_{s_{1}(t)}\left(f_{1} \omega_{g+n}(t)\right) \\
\vdots & \ddots & \vdots & \\
\operatorname{Res}_{s_{1}(t)}\left(f_{g_{1}} \omega_{1}(t)\right) & \operatorname{Res}_{s_{1}(t)}\left(f_{g_{1}} \omega_{2}(t)\right) & \cdots & \operatorname{Res}_{s_{1}(t)}\left(f_{g_{1}} \omega_{g+n}(t)\right) \\
\operatorname{Res}_{s_{1}(t)}\left(f_{g_{1}+1} \omega_{1}(t)\right) & \operatorname{Res}_{s_{1}(t)}\left(f_{g_{1}+1} \omega_{2}(t)\right) & \cdots & \operatorname{Res}_{s_{1}(t)}\left(f_{g_{1}+1} \omega_{g+n}(t)\right) \\
\vdots & \ddots & \vdots & \\
\operatorname{Res}_{s_{1}(t)}\left(f_{g} \omega_{1}(t)\right) & \operatorname{Res}_{s_{1}(t)}\left(f_{g} \omega_{2}(t)\right) & \cdots & \operatorname{Res}_{s_{1}(t)}\left(f_{g} \omega_{g+n}(t)\right)
\end{array}\right|\right) .
\end{aligned}
$$

Now it is easy to see that the last expression has a meaning when $t$ goes to 0 .

Hence $\lim _{t \rightarrow 0}\left\langle\omega\left(\mathfrak{X}_{t},\{\alpha(t), \beta(t)\}\right) \mid v_{1} \otimes v_{2}\right\rangle$ always exist.

QED

\section{References}

[AU2] J. Andersen \& K. Ueno, Geometric construction of modular functors from conformal field theory, MPS-preprint 2003 - 5. math.QA/0304135.

[AU3] J. E. Andersen \& K. Ueno, Construction of the Reshetikhin-Turaev TQFT from conformal field theory, Preprint in preparation.

[BPZ] A. A. Belavin, A. M. Polyakov \& A. B. Zamolodchikov, Infinite conformal symmetry in two-dimensional quantum field theory, Nucl. Phys.B241 (1984), 338 380.

[F] J. D. Fay, Theta functions on Riemann surfaces, Lecture Notes in Math. 352 (1973), Springer-Verlag.

[FP] L. D. Faddeev \& V. N. Popov, Feynman diagrams for the Yang-Mills fields, Physics Letters, B25 (1967), 29 - 30.

[KNTY] N. Kawamoto, Y. Namikawa, A. Tsuchiya \& Y. Yamada, Geometric realization of conformal field theory on Riemann surfaces, Commun. Math. Phys.,116 (1988), $247-308$.

[KSUU] Kuroda, Y. Shimizu, Uematsu \& K. Ueno, Abelian conformal field theory under degenerations of curves, Preprint, 2002.

[SA] M. Sato, Soliton equations as dynamical systems on an infinite dimensional Grassmann manifold, Res. Inst. Math. Sci., Kyoto Univ.- Kokyuroku 439, 30 46.

[TK] A. Tsuchiya and Y. Kanie, Vertex operators in conformal field theory on $\mathbf{P}^{1}$ and monodromy representations of braid group, Advanced Studies in Pure Math., 16 (1988), 297 - 372, Erratum, ibid., 19 (1989), 675 - 682. 
[TUY] A.Tsuchiya, K. Ueno \& Y. Yamada Conformal field theory on universal family of stable curves with gauge symmetries, Adv. Stud. in Pure Math., 19 (1989), 459-566

[U1] K. Ueno, On conformal field theory, London Math. Soc. Lecture Notes Series 208 (1995), 283 - 345, Cambridge Univ. Press.

[U2] K. Ueno, Introduction to conformal field theory with gauge symmetry, Lecture notes in pure and applied mathematics 184, Geometry and Physics, 603 - 745, Marcel Dekker, 1996. 